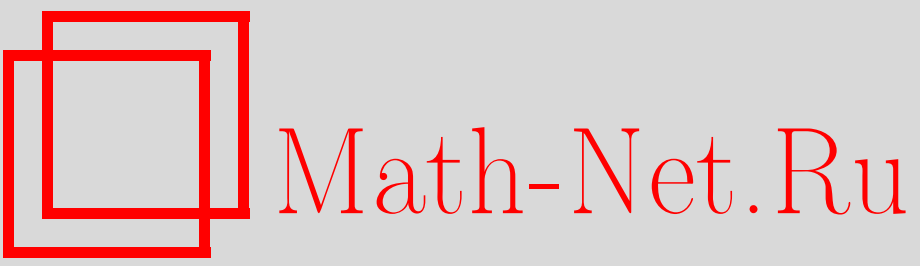

Э. Б. Винберг, Коммутативные однородные пространства и коизотропные симплектические действия, УМН, 2001, том 56, выпуск 1, 3-62

DOI: https://doi.org/10.4213/rm356

Использование Общероссийского математического портала Math-Net.Ru подразумевает, что вы прочитали и согласны с пользовательским соглашением

http://www . mathnet.ru/rus/agreement

Параметры загрузки:

IP: 3.89 .197 .203

26 апреля 2023 г., 12:31:18 


\section{КОММУТАТИВНЫЕ ОДНОРОДНЫЕ ПРОСТРАНСТВА И КОИЗОТРОПНЫЕ СИМПЛЕКТИЧЕСКИЕ ДЕЙСТВИЯ}

\section{Э. Б. ВИНБЕРГ}

Статья посвящена обзору связей между следуюшими свойствами риманова однородного пространства $X=G / K$ : слабая симметричность в смысле А. Сельберга, коммутативность алгебры $K$-инвариантных мер на $X$, коммутативность алгебры $G$-инвариантных дифференциальных операторов на $X$, коммутативность алгебры Пуассона $G$-инвариантных функций на кокасательном расслоении пространства $X$ и (в случае редуктивной группы $G$ ) простота спектра линейного представления группы $G$ в алгебре полиномиальных функций на $X$. Приводятся различные структурные и классификационные резулттаты, в том числе полученная автором классификация неприводимых римановых однородных пространств гейзенбергова типа, для которых алгебра Пуассона инвариантных функций на кокасательном расслоении коммутативна.

Библиография: 49 названий.

\section{СОДЕРЖАНИЕ}

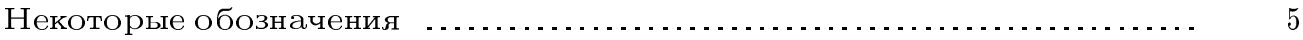

Глава І. Коммутативные однородные пространства .................. 6

$\S 1$. Симмет рические и слабо симметрические пространства .......... 6

$\S 2$. Пары Гельфанда и коммутативные однородные пространства ...... 11

$\S 3$. Сферические однородные пространства редуктивных групп ........ 18

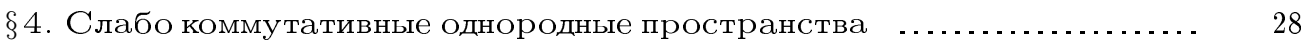

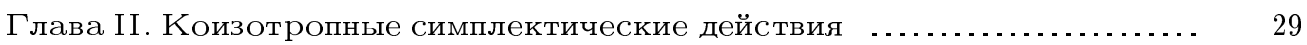

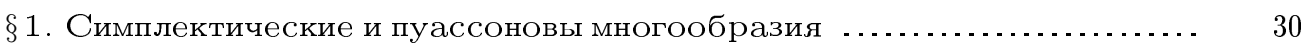

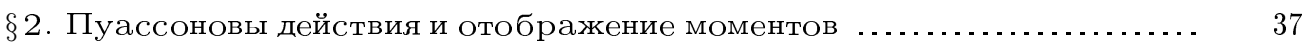

$\S 3$. Коранг симплектического действия .......................... 42

$\S 4$. Структура слабо коммутативных однородных пространств ........ 51

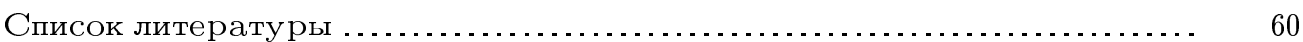

Одной из целей этой статьи является собрание воедино информации о связях между некоторыми замечательными классами римановых однородных пространств - информации, рассьпанной доселе во многих публикациях разных авторов.

Статья написана при поддержке гранта РФФИ 98-01-00598, гранта INTAS 97-1570, а также гранта CRDF RM1-2088. Работа над статьей была завершена во время моего пребывания в гостеприимном университете Билефельда, которому я выражаю свою глубокую благодарность. 
Пусть $X=G / K$ - связное риманово однородное пространство группы Ли $G$. Пространство $X$ назьвается коммутативным (или пара $(G, K)$ назьвается парой Гель$\oint a н \partial a)$, если выполнены следуюшие эквивалентные условия:

1) алгебра $K$-инвариантных мер с компактным носителем на $X$ коммутативна (относительно свертки);

2 ) алгебра $G$-инвариантных дифференциальных операторов на $X$ коммутативна.

$\mathrm{K}$ числу коммутативных пространств относятся все симметрические пространства и, более обшо, все так назьваемые слабо симметрические пространства. Пространство $X$ назьвается слабо симметрическим, если сушествует такой диффеоморфизм $s$ многообразия $X$, нормализуюший групп $G$, что для любых двух точек $x, y \in X$ в смежном классе $s G$ имеется преобразование, переставляющее $x$ и $y$.

Если группа $G$ редуктивна, то условия 1$)$ и 2 ) эквивалентны также любому из следуюших условий:

3) пространство $X$ слабо симметрично;

4) естественное линейное представление групшы $G$ в алгебре $\mathbb{C}[X]$ полиномиальных функщий на $X$ имеет простой спектр;

5) алгебра Пуассона $G$-инвариантных функций на кокасательном расслоении $T^{*} X$ многообразия $X$ коммутативна.

Аффинные однородные пространства комплексных редуктивных алгебраических групп, удовлетворяюшие условию 4), изучались в работах по алгебраическим группам преобразований под названием (аффинных однородных) сферических многообразий. В частности, известна их классификация. Коммутативные римановы однородные пространства редуктивных групп Ли - это их вешественные формы.

В общем случае условие 5) следует из условия 2). Римановы однородные пространства, удовлетворяюшие условию 5), мы назьваем в этой статье слабо коммутативнылми. Впрочем, весьма правдоподобно, что условие 5) эквивалентно условию 2), т.е. всякое слабо коммутативное пространство на самом деле коммутативно. Во всяком случае, это верно в двух противоположных случаях: когда группа $G$ редуктивна и когда $G=N \lambda K$, где $N$ - нильпотентная группа Ли.

Однородное пространство вида $X=(N \lambda K) / K$ может быть коммутативньм, только если группа $N$ не более чем 2 -ступенно нильпотентна, т.е. ее коммутант $Z$ содержится в ее центре. Такие однородные пространства мы называем пространствами гейзенбергова типа. Если при этом естественное линейное представление группы $K$ в векторном пространстве $V=N / Z$ неприводимо, то мы называем пространство $X$ неприводимыл. В статье приведена классификация всех неприводимых коммутативных однородных пространств гейзенбергова типа. Ранее эта классификация была известна для случая, когда $\operatorname{dim} Z=1$. (Случай $\operatorname{dim} Z=0$ тривиален.)

Во второй части статьи (слабо) коммутативные однородные пространства обсуждаются в более широком контексте эквивариантной симплектической геометрии.

Корангом симплектического действия (вещественной или комплексной) группы Ли $G$ на связном симплектическом (вешественном или комплексном) гладком многообразии $M$ назьвается коранг касательного пространства орбиты общего положения. Симплектические действия коранга 0 назьваются коизотропными.

Однородное пространство $X=G / K$ слабо коммутативно тогда и только тогда, когда действие $G: T^{*} X$ коизотропно (в смысле стандартной симплектической структуры кокасательного расслоения). 
Пусть задано любое действие редуктивной комплексной алгебраической группы $G$ на неприводимом комплексном алгебраическом многообразии $X$. В этой ситуации оказьвается, что коранг действия $G: T^{*} X$ просто выражается в терминах действия $G: X$. А именно, пусть $B$ - борелевская подгруппа группы $G$. Тогда коразмер-

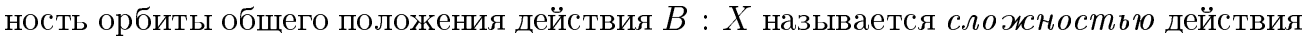
$G: X$. В теории редуктивных алгебраических групп преобразований сложность является весьма важной и хорошо изученной характеристикой действия. Оказьвается, что коранг действия $G: T^{*} X$ равен удвоенной сложности действия $G: X$. В частности, действие $G: T^{*} X$ коизотропно тогда и только тогда, когда действие $G: X$ имеет сложность 0 или, как говорят, является сферическим. В статье дается новое доказательство этих фактов в случае, когда многообразие $X$ квазиаффинно.

По поводу используемых в статье стандартных сведений из теории групп Ли и алгебраических групп мы отсылаем читателя к книге [1], а по поводу сведений из теории инвариантов - к обзорной статье [2], хотя все это, конечно, может быть найдено и во многих других местах.

Необходимые ссылки на оригинальные работы, содержашие упомянутые в этом резюме и другие результаты, даны в теле статьи.

Я благодарю Г. И. Ольшанского за полезные замечания и Д. А. Титашева, указавшего мне на некоторые неточности в предыдущем варианте статьи.

\section{Некоторые обозначения}

$\langle M\rangle$ - линейная оболочка подмножества $M$ векторного пространства $V$.

$U^{\circ}$ - аннулятор подпространства $U \subset V$ в сопряженном (= дуальном) пространстве $V^{*}$.

$S(V)=\bigoplus_{n=0}^{\infty} S^{n}(V)$ - симметрическая алгебра пространства $V$.

$\Lambda(V)=\bigoplus_{n=0}^{\infty} \Lambda^{n}(V)$ - внешняя алгебра пространства $V$.

$P V$ - проективное пространство, ассоциированное с пространством $V$.

$\mathrm{L}(V)$ - алгебра линейных операторов в пространстве $V$.

$\mathrm{GL}(V)$ - группа невырожденных линейных операторов в пространстве $V$.

$\mathrm{O}(V)$ - ортогональная группа пространства $V$ (относительно заданной квадратичной формы).

$X^{G}$ - подмножество неподвижных точек группы $G$, действуюшей на множестве $X$.

$\widetilde{G}$ - отношение эквивалентности, определяемое действием группы $G$.

$\underset{H}{G} * X$ - однородное расслоение над однородньм пространством $G / H$, определяемое действием $H: X$, т.е. фактор прямого произведения $G \times X$ по действию группы $H$, определяемому формулой $h(g, x)=\left(g h^{-1}, h x\right)$.

$N \lambda H$ - полупрямое произведение групп $N$ и $H$ (в котором подгруппа $N$ нормальна).

$N(H)$ - нормализатор подгруппы $H$ в группе.

$Z(H)$ - централизатор подгруппы $H$ в группе.

$T_{x}(X)$ - касательное пространство многообразия $X$ в точке $x$.

$F[X]$ - алгебра регулярных (полиномиальных) функций на алгебраическом многообразии $X$ над полем $F$.

$F(X)$ - поле рациональных функций на неприводимом алгебраическом многообразии $X$.

$G^{0}-$ связная компонента единицы группы Ли $G$.

$\exp : \mathfrak{g} \rightarrow G$ - экспоненщиальное отображение в группе Ли $G$. 
$g \xi \in T_{g}(G)$ - результат левого сдвига элемента $\xi \in \mathfrak{g}=T_{e}(G)$ на элемент $g$ групшы Ли $G$.

$\mathfrak{n} \rightarrow \mathfrak{h}-$ полупрямая сумма алгебр Ли $\mathfrak{n}$ и $\mathfrak{h}$ (в которой $\mathfrak{n}-$ идеал).

$\mathfrak{n}(\mathfrak{h})$ - нормализатор подалгебры $\mathfrak{h}$ в алгебре Ли.

$\mathfrak{z}(\mathfrak{h})$ - централизатор подалгебры $\mathfrak{h}$ в алгебре Ли.

Групш Ли обозначаются прописными латинскими буквами, а их касательные алгебры Ли - соответствуюшими строчными готическими буквами.

\section{Глава I. Коммутативные однородные пространства}

В этой главе мы рассматриваем однородные пространства вида $X=G / K$, где $G$ - (вешественная) группа Ли и $K$ - ее компактная подгруппа. Если не оговорено противное, то мы считаем, что многообразие $X$ связно и действие $G: X$ эффективно, т.е. $K$ не содержит нетривиальных нормальных подгрупп группы $G$. "Базисная" точка $e K \in X$ обозначается буквой $o$.

Пространства этого типа обладают инвариантной римановой метрикой (вообще говоря, не единственной). Однако мы рассматриваем их именно как однородные пространства, а не как римановы многообразия. Это, в частности, означает, что изометричные римановы многообразия с различными транзитивными группами движений рассматриваются как неизоморфные объекты. Такая точка зрения, отличная от принятой в большинстве геометрических работ, более удобна в контексте этой статьи.

\section{$\S$ 1. Симметрические и слабо симметрические пространства}

1. Понятие симметрического пространства было введено Э. Картаном в 1926 г. Существует несколько эквивалентных определений симметрического пространства. Начнем с того из них, которое более удобно для нас.

ОПРЕДЕЛЕНИЕ 1. Однородное пространство $X=G / K$ назьвается симметрическим, если сушествует такой диффеоморфизм $s$ многообразия $X$, что $s G s^{-1}=G$, $s o=о$ и

$$
d s(\xi)=-\xi \quad \forall \xi \in T_{o}(X)
$$

Диффеоморфизм $s$, удовлетворяющий условиям этого определения, автоматически инволютивен, т.е. $s^{2}=\mathrm{id}$. Он порождает инволютивньй автоморфизм $\sigma$ группы $G$ по формуле

$$
\sigma(g)=s g s^{-1}
$$

Если $G^{\sigma}$ - подгруппа неподвижных точек этого автоморфизма, то

$$
\left(G^{\sigma}\right)^{0} \subset K \subset G^{\sigma}
$$

Обратно, если сушествует инволютивный автоморфизм $\sigma$ группы $G$, удовлетворяющий условию (3) или эквивалентному ему условию

$$
\mathfrak{g}^{\sigma}=\mathfrak{k},
$$

то диффеоморфизм $s$ многообразия $X$, определяемьй по формуле

$$
s(g o)=\sigma(g) o,
$$


удовлетворяет условиям определения 1 и, следовательно, однородное пространство $X=G / K$ является симметрическим.

Структура симметрических пространств хорошо известна (см., например, [3]). Напомним лишь один факт: всякое симметрическое пространство локально изоморфно прямому произведению симметрического пространства полупростой группы Ли и евклидова пространства. Под евклидовьм пространством при нашей точке зрения здесь следует понимать однородное пространство вида $(V \lambda K) / K$, где $V$-векторная группа, а $K$ - любая замкнутая подгруппа групшы $\mathrm{O}(V)$ (так что имеется много евклидовых пространств одной размерности).

2. В 1956 г. А. Сельберг в своей знаменитой работе [4], посвященной так назьваемой “формуле следа”, ввел понятие слабо симметрического пространства. Нам будет удобно следуюшее определение слабо симметрического пространства, отличное от оригинального определения Сельберга, но эквивалентное ему с точностью до одной мелочи, о которой будет сказано ниже. Оригинальное определение также будет дано ниже.

ОПРЕДЕЛЕНИЕ 2. Однородное пространство $X=G / K$ назьвается слабо симметрическим, если сушествует такой диффеоморфизм $s$ многообразия $X$, что $s G s^{-1}=G, s о=$ ои

$$
d s(\xi) \underset{K}{\sim}-\xi \forall \xi \in T_{o}(X) .
$$

Из этого определения ясно, что всякое симметрическое пространство является слабо симметрическим.

В отличие от диффеоморфизма $s$ в определении симметрического пространства, диффеоморфизм $s$ в этом определении определен не однозначно: как минимум его можно умножить на любой элемент из $K$.

Приведем несколько примеров слабо симметрических пространств, не являющихся симметрическими.

ПримеР 1. Оригинальньй пример Сельберга - 3-мерное пространство

$$
X=\left(\mathrm{PSL}_{2}(\mathbb{R}) \times \mathrm{SO}_{2}\right) / \mathrm{SO}_{2}
$$

единичных касательных векторов плоскости Лобачевского $L^{2}$. Здесь множитель $\mathrm{PSL}_{2}(\mathbb{R})$ - группа собственных движений плоскости $L^{2}$, действующая на $X$ естественным образом, множитель $\mathrm{SO}_{2}$ - группа одновременных поворотов всех касательных векторов на один и тот же угол. Стабилизатор $K=\mathrm{SO}_{2}$ базисной точки $o \in X$, вложенньй в произведение антидиагональным образом, тривиально действует на слое естественного расслоения $X \rightarrow L^{2}$, проходящем через $о$. Соответственно этому он действует в касательном пространстве $T_{o}(X)$ поворотами вокруг “вертикальной" оси, касающейся этого слоя. Возьмем в качестве $s$ диффеоморфизм пространства $X$, индуцированньй отражением плоскости $L^{2}$ относительно прямой, направляющий вектор которой есть точка $o$ пространства $X$. Тогда $d_{o} s$ - это поворот на $\pi$ вокруг прямой, ортогональной вертикальной оси, и нетрудно видеть, что условие (6) вьполнено. 
ПримеР 2. Вырождением предыдушего пространства является так назьваемое нильпространство Тёрстона

$$
X=\left(H 入 \mathrm{SO}_{2}\right) / \mathrm{SO}_{2},
$$

где $H$ - 3-мерная группа Гейзенберга (изоморфная группе унитреугольных матриц 3 -го порядка). Пространство $X$ естественньм образом отождествляется с группой $H$; при этом пространство $T_{o}(X)$ отождествляется с касательной алгеброй h группы $H$. Группа $K=\mathrm{SO}_{2}$ действует на $T_{o}(X)$ поворотами вокруг прямой $\mathfrak{z}$, являющейся центром алгебры $\mathfrak{h}$. В качестве $s$ можно взять автоморфизм группы $H$, диффференциал которого есть поворот на $\pi$ относительно некоторой прямой, ортогональной $\mathfrak{z}$. Условие (6) будет вьполнено по той же причине, что и в предыдущем примере.

ПРИмеР 3. Рассмотрим однородное пространство

$$
X=\left(\mathrm{SO}_{3} \times \mathrm{SO}_{3} \times \mathrm{SO}_{3}\right) / \mathrm{SO}_{3},
$$

где стабилизатор $K=\mathrm{SO}_{3}$ вложен в произведение диагональным образом. Касательное пространство $T_{o}(X)$ может быть отождествлено с прямой суммой двух копий евклидова пространства $\mathbb{E}^{3}$, на которой группа $K=\mathrm{SO}_{3}$ действует естественным образом. Так как любые два вектора пространства $\mathbb{E}^{3}$ можно одновременно обратить поворотом на $\pi$ вокруг ортогональной им оси, то условие (6) выполнено для $s=\mathrm{id}$.

ПримеР 4. Сфера $S^{2 n-1}$ может рассматриваться как однородное пространство групшы $\mathrm{SU}_{n}$ :

$$
S^{2 n-1}=\mathrm{SU}_{n} / \mathrm{SU}_{n-1} \subset \mathbb{C}^{n}
$$

В этом качестве она, не будучи симметрическим пространством, все же является слабо симметрическим пространством. В качестве $s$ можно взять комплексное сопряжение.

3. Приведем еще два эквивалентных определения слабой симметричности.

ПРЕДЛОЖЕНИЕ 1. Однородное пространство $X=G / K$ слабо симметрично тогда и только тогда, когда выполнено любое из следующих әквивалентных условий:

(WS1) существует такой диффеоморфизм s многообразия $X$, что $s G s^{-1}=G u$

$$
(s x, s y) \underset{G}{\widetilde{G}}(y, x) \quad \forall x, y \in X
$$

(WS2) существует такой автоморфизм $\sigma$ группь $G$, что

$$
\sigma(g) \in K g^{-1} K \quad \forall g \in G
$$

Условие (WS1) является оригинальным определением Сельберга, с той оговоркой, что он дополнительно требовал, чтобы $s^{2} \in G$. Мне кажется более удобньм не требовать этого; с другой стороны, я не знаю примеров слабо симметрических пространств в смысле этой статьи, для которых это условие не было бы выполнено при подходяшем выборе $s$. 
ДокАЗАТЕльСтво. Будем считать, что многообразие $X$ снабжено $G$-инвариантной римановой метрикой. При вьполнении любого из условий (6) и (7) эта метрика будет автоматически инвариантна и относительно $s$.

Пусть $s$ - диффеоморфизм многообразия $X$, нормализуюший группу $G$. Тогда $s$ сохраняет отношение $G$-эквивалентности на $X \times X$, и из выполнения условия (7) для какой-то пары $(x, y) \in X \times X$ следует его вьполнение для любой эквивалентной пары.

Предположим, что $s o=o$ и вьполнено условие (6). Проверим вьполнение условия (7). Соединим точки $x$ и $y$ геодезическим отрезком $\overline{x y}$. Пусть $p-$ середина этого отрезка. Заменив пару $(x, y)$ на эквивалентную, можно считать, что $p=o$. Пусть $\xi-$ единичный касательный вектор отрезка $\overline{x y}$ в точке $o$, и пусть $k \in K$ - такой элемент, что $d s(\xi)=-d k(\xi)$. Тогда $s x=k y$ и $s y=k x$ (см. рис. 1$)$.

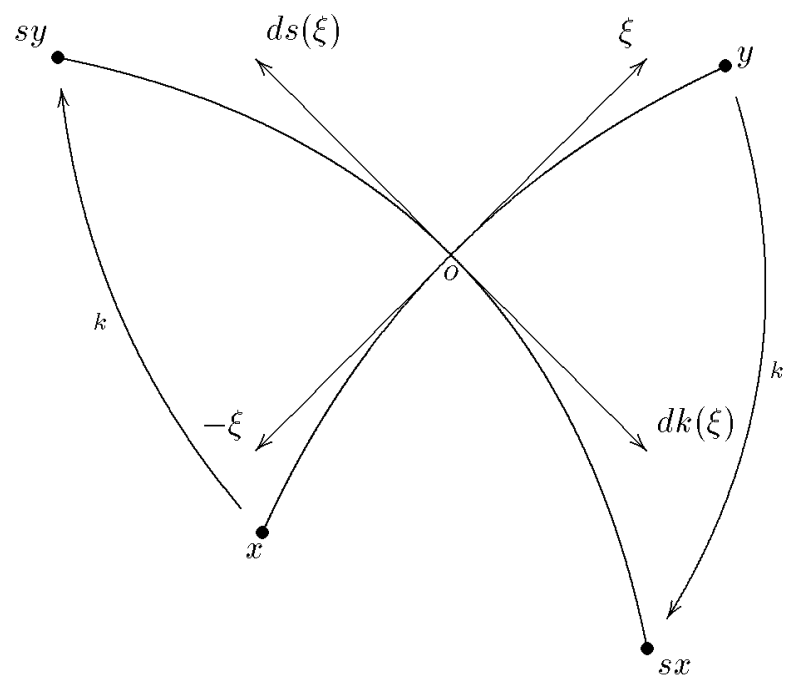

Рис. 1

Обратно, пусть вьполнено условие (7). Домножив $s$ на подходяший элемент группы $G$, можно считать, что $s o=o$. Проверим выполнение условия (6). Пусть $\xi \in T_{o}(X)$ - любой единичньй вектор. Проведем через точку о геодезическую по направлению $\xi$ и возьмем на ней точки $x$ и $y$ на равном расстоянии от $o$, как на рис. 1 . Будем считать, что точки $x$ и $y$ настолько близки к $o$, что отрезок $\overline{x y}$ нашей геодезической с серединой в точке $о$ является единственньм кратчайшим геодезическим отрезком, соединяющим $x$ и $y$. Тогда $s(\overline{x y})$ будет единственным кратчайшим геодезическим отрезком, соединяюшим $s x$ и $s y$. Пусть $g \in G$ - такой элемент, что $g x=s y$ и $g y=s x$. Тогда $g(\overline{x y})=s(\overline{y x})$ и, следовательно, $g o=o$, т.е. $g \in K$, и $d g(\xi)=-d s(\xi)$.

Таким образом, мы доказали, что условие (WS1) эквивалентно слабой симметричности пространства $X$.

Пусть снова $s$ удовлетворяет условию (7) и $s o=o$. Пусть $\sigma-$ автоморфизм группы $G$, определяемый по формуле (5). Тогда для любого $g \in G$

$$
(o, \sigma(g) o)=(s o, s g o) \underset{G}{\widetilde{f}}(g o, o),
$$


т.е. сушествует такой элемент $h \in G$, что $h g o=o$ и $h o=\sigma(g) o$. Из первого из этих равенств следует, что $h=k_{1} g^{-1}\left(k_{1} \in K\right)$. Подставляя это во второе равенство, получаем, что

$$
\sigma(g)=h k_{2}=k_{1} g^{-1} k_{2} \quad\left(k_{1}, k_{2} \in K\right) .
$$

Таким образом, для автоморфизма $\sigma$ выполнено условие (8).

Обратно, пусть автоморфизм $\sigma$ группы $G$ удовлетворяет условию (8). Полагая $g \in K$, получаем, что $\sigma(K)=K$. Пусть $s-$ диффеоморфизм многообразия $X$, определяемьй по формуле (2). Проверим вьполнение условия (7). Заменив пару $(x, y)$ на эквивалентную, можно считать, что $x=o, y=g o(g \in G)$. Пусть $\sigma(g)=k_{1} g^{-1} k_{2}$ $\left(k_{1}, k_{2} \in K\right)$. Тогда, обрашая предыдушие выкладки, получаем, что

$$
(s x, s y)=(o, \sigma(g) o) \underset{G}{\sim}(g o, o)=(y, x) .
$$

4. Понятие слабо симметрического пространства, введенное Сельбергом, кажется, было забыто на 40 лет. Лишш около 1995 г. геометры начали изучать эти пространства (см. [5]-[10]). При этом они выработали следуюшее определение, отличное от определения Сельберга, но более естественное с точки зрения геометрии: слабо симметрическим пространством называется связное риманово многообразие $X$, для любых двух точек которого существует движение, их переставляющее. В терминологии настоящей статьи это означает, что $X$ является слабо симметрическим пространством относительно полной группы движений. Как мы сейчас покажем, в этом случае оно является слабо симметрическим пространством и относительно связной компоненты этой группы.

Лемма 1. Пусть $K \subset \mathrm{GL}(V)$ - компактная линейная группа и $s_{0} \in \mathrm{GL}(V)$ такой линейный оператор, что $s_{0} K s_{0}^{-1}=K u$

$$
s_{0}(\xi) \underset{K}{\sim}-\xi \quad \forall \xi \in V .
$$

Тогда найдется такой әлемент $s \in K s_{0}$, что

$$
s(\xi) \underset{K^{0}}{\sim}-\xi \quad \forall \xi \in V .
$$

ДОКАЗАТЕЛЬСТВо. Для всякого $s \in K s_{0}$ положим

$$
V(s)=\left\{\xi \in V: s(\xi) \underset{K^{0}}{\sim}-\xi\right\} \subset V .
$$

Так как орбиты компактной линейной групшы разделяются полиномиальньми инвариантами [1], то $V(s)$ - алгебраическое многообразие. Очевидно, что $V(s)$ зависит только от смежного класса по $K^{0}$, в котором лежит $s$, и, стало быть, имеется лишш конечное число таких многообразий. Из условия (9) следует, что пространство $V$ ими покрьвается; но тогда оно должно совпадать с одним из них.

Таким образом, в определении 2 слабо симметрического пространства эквивалентность относительно подгрупшы $K$ можно заменить эквивалентностью относительно ее связной компоненты $K^{0}$. Отсюда, в частности, следует, что если однородное пространство $G / K$ является слабо симметрическим, то и пространство $G^{0} /\left(K \cap G^{0}\right)$ (т.е. то же многообразие, но с группой $G^{0}$ ) является таковым.

Для однородных пространств связных групп Ли мы докажем, что свойство слабой симметричности является локальным, т.е. зависит только от алгебры Ли $\mathfrak{g}$ и ее подалгебры k. 
ПРЕДЛОЖЕНИЕ 2. Пусть $X=G / K$ - слабо симметрическое пространство. Тогда группа $N(K) / K$ абелева и автоморфизм $\sigma$ действует на ней как инверсия.

ДокАЗАТЕЛЬство. Первое утверждение следует из второго, а второе - из свойства (8).

СледСтВИЕ. Автоморфизм $\sigma$ действует на иентре $Z$ группь $G$ как инверсия.

ПРЕДЛОЖЕНИЕ 3. Однородное пространство связной группь Ли является слабо симметрическим тогда и только тогда, когда таковым является его односвязное накрывающее пространство.

ДоКАЗАТЕльство. Если допустить неэффективные (но локально эффективные) действия, то односвязное накрытие $p: X \rightarrow X_{1}$ однородного пространства $X_{1}$ связной групшы Ли может быть представлено следуюшим образом:

$$
p: X=G / K \rightarrow G / \widehat{K}=X_{1}, \quad g K \mapsto g \widehat{K},
$$

где подгрупа $\widehat{K}$ (не обязательно компактная) содержит подгруппу $K$ в качестве связной компоненты. Можно считать действие $G: X$ эффективным; тогда ядром неэффективности действия $G: X_{1}$ будет подгруппа $N=\widehat{K} \cap Z$, где $Z$ - центр группы $G$. Положим $G_{1}=G / N$ и $K_{1}=\widehat{K} / N$; тогда $X_{1}=G_{1} / K_{1}$ будет эффективным представлением однородного пространства $X_{1}$.

Если пространство $X_{1}$ слабо симметрично, то по лемме 1 соответствуюший дифофеоморфизм $s_{1}$ может быть выбран так, чтобы условие (6) было вьполнено для группы $K_{1}^{0}=K$. Он может быть (единственным образом) поднят до диффеоморфизма $s$ многообразия $X$, оставляюшего на месте точку $о$. При этом $s$ будет нормализовать группу $G$ и будет выполнено условие (6).

Обратно, пусть пространство $X$ слабо симметрично и $\sigma$-автоморфизм групшы $G$, порождаемьй соответствуюшим диффеоморфизмом $s$. Так как $\widehat{K} \subset N(K)$, то из предложения 2 следует, что $\sigma(\widehat{K})=\widehat{K}$ и, значит, автоморфизм $\sigma$ спускается до автоморфизма $\sigma_{1}$ группы $G_{1}$, сохраняюшего подгрупп $K_{1}$. Так как $K_{1} \supset K$, то соответствующий диффеоморфизм $s_{1}$ многообразия $X_{1}$ удовлетворяет условию $(6)$.

\section{§. Пары Гельфанда и коммутативные однородные пространства}

1. Пусть $G$ - конечная групша и $\mathbb{R} G$ - ее вещественная групповая алгебра. Для любой подгрупшы $K \subset G$ подпространство $(\mathbb{R} G)^{K \times K}$ элементов алгебры $\mathbb{R} G$, инвариантных относительно умножений слева и справа на элементы подгрупш $K$, является подалгеброй. Пара $(G, K)$ назьвается парой Гельфанда, если эта подалгебра коммутативна. Это равносильно тому, что в пространстве любого неприводимого комплексного линейного представления группы $G$ подпространство $K$-инвариантных векторов не более чем одномерно, или, по двойственности Фробениуса, тому, что представление группы $G$ в пространстве $\mathbb{C}[G / K]$ функций на $G / K$ имеет простой спектр (см., например, [11]).

Все это может быть обобщено на локально компактные топологические групшы, если принять какую-либо топологическую версию групоовой алгебры.

Пусть $G$ - произвольная локально компактная топологическая группа, и пусть $C(G)$ - пространство непрерывных функций на $G$ с топологией компактной сходимости. Назовем групповой алгеброй группы $G$ и обозначим через $\mathbb{R} G$ пространство 
непрерьвных линейных функционалов на $C(G)$, т.е. обобщенных мер с компактньм носителем на $G$, с операцией свертки $*$ в качестве умножения.

Пусть $K \subset G$ - компактная подгруппа.

Отметим, что пространство $(\mathbb{R} G)^{K \times K}$ может быть отождествлено с пространством непрерывных линейных функционалов на $C(G)^{K \times K}$.

ОПРедЕЛЕниЕ 3. Пара $(G, K)$ называется парой Гельфанда, если алгебра $(\mathbb{R} G)^{K \times K}$ коммутативна.

Известно [12] (но нам это не понадобится), что это условие равносильно тому, что в пространстве любого неприводимого унитарного представления групшы $G$ подпространство $K$-инвариантных векторов не более чем одномерно, или тому, что представление группы $G$ в пространстве $L^{2}(G / K)$ имеет простой спектр.

2. Любой автоморфизм $\sigma$ групшы $G$ индуцирует естественным образом автоморфизм ее групповой алгебры $\mathbb{R} G$. При этом если $\sigma(K)=K$, то подалгебра $(\mathbb{R} G)^{K \times K}$ инвариантна относительно $\sigma$. Аналогично, любой антиавтоморфизм групшы $G$ (в частности, инверсия) индуцирует антиавтоморфизм алгебры $\mathbb{R} G$.

ПрЕДЛОЖЕНИЕ 4. Предположим, что существует такой автоморфизм $\sigma$ группьь $G$, что

$$
\sigma(g) \in K g^{-1} K \quad \forall g \in G .
$$

Тогда пара $(G, K)$ является парой Гельфанда.

ДоКАЗАТЕЛЬСТво. Из условия (11) следует, в частности, что $\sigma(K)=K$, так что $\sigma$ индуцирует автоморфизм алгебры $(\mathbb{R} G)^{K \times K}$. В то же время из (11) следует, что этот автоморфизм совпадает с антиавтоморфизмом, индуцированным инверсией. Значит, алгебра $(\mathbb{R} G)^{K \times K}$ коммутативна.

Заметим, что условие (11) совпадает с условием (8) предложения 1.

СЛЕДСТВИЕ. Если $G / K$ - слабо симметрическое пространство, то $(G, K)$ пара Гельфанда.

Условие (11) иногда назьвают условием Гельфанда, так как на нем было основано предложенное И. М. Гельфандом (см. [13], [12]) доказательство того, что для всякого симметрического пространства $G / K$ компактной групш Ли $G$ представление групшы $G$ в пространстве $L^{2}(G / K)$ имеет простой спектр - факта, доказанного ранее $Э$. Картаном путем перебора случаев.

Легко видеть, что если $N$ - нормальная подгруппа группы $G$, содержашаяся в $K$, то пара $(G, K)$ является парой Гельфанда тогда и только тогда, когда таковой является пара $(G / N, K / N)$. Это означает, что свойство пары $(G, K)$ быть парой Гельфанда есть на самом деле свойство однородного пространства $G / K$.

3. Для любой меры $\mu \in \mathbb{R} G$ обозначим через $\mu^{\natural}$ результат ее двустороннего усреднения по группе $K$. Через $\delta_{g}$ обозначим $\delta$-функцию точки $g \in G$.

ПреДЛОЖенИЕ 5. Пара $(G, K)$ является парой Гельфанда тогда и только тогда, когда меры $\delta_{g}^{\natural}$ и $\delta_{h}^{\natural}$ коммутируют для любых $g, h \in G$. Более того, если $G$ - группа Ли и многообразие $X=G / K$ связно, то достаточно потребовать, чтобы меры $\delta_{g}^{\natural}$ и $\delta_{h}^{\natural}$ коммутировали для любых $g, h$ из некоторой окрестности единицы группь $G$. 
ДокАЗАТЕльство. Известно (см., например, [14]), что линейные комбинации $\delta$ функций, т.е. меры с конечньм носителем, образуют плотное подмножество в алгебpe $\mathbb{R} G$ в слабой топологии. С ледовательно, линейные комбинации их усреднений образуют плотное подмножество в алгебре $(\mathbb{R} G)^{K \times K}$. Так как операция свертки непрерьвна по каждому множителю в слабой топологии (см. там же), то из перестановочности мер $\delta_{g}^{\natural}$ и $\delta_{h}^{\natural}$ для любых $g, h \in G$ вытекает коммутативность алгебры $(\mathbb{R} G)^{K \times K}$.

Пусть теперь $G$ - группа Ли. Интеграл функции $\varphi \in C(G)^{K \times K}$ по мере $\delta_{g}^{\natural} * \delta_{h}^{\natural}$ равен

$$
I(\varphi ; g, h)=\int_{K} \varphi(g k h) d k,
$$

где $d k$ обозначает нормированную меру Хаара на группе $K$. Следовательно, если $\varphi$ - аналитическая функщия, то $I(\varphi ; g, h)$ - аналитическая функция от $g$ и $h$, и если многообразие $X=G / K$ связно и равенство $I(\varphi ; g, h)=I(\varphi ; h, g)$ имеет место для всех $g$ и $h$, достаточно близких к единице группы $G$, то оно имеет место вообще для всех $g$ и $h$. Но так как множество аналитических функций на групе $G$ (как и на всяком вешественном аналитическом многообразии) плотно в $C(G)[15]$, то из выполнения этого равенства для всех аналитических функщий $\varphi$ следует, что $\delta_{g}^{\natural} * \delta_{h}^{\natural}=\delta_{h}^{\natural} * \delta_{g}^{\natural}$.

4. В случае, когда $G$ - линейная группа Ли, понятие пары Гельфанда можно перевести в алгебраическую плоскость.

Пусть $G \subset \mathrm{GL}(U)$ - линейная группа Ли и $G^{a}$ - ее замькание в топологии Зарисского в $\mathrm{GL}(U)$. Назовем алгеброй полиномиальных функиий на $G$ и обозначим через $\mathbb{R}[G]$ алгебру функций на групе $G$, являюшихся ограничениями функций из $\mathbb{R}\left[G^{a}\right]$. Алгебра $\mathbb{R}[G]$ порождается матричньми элементами тавтологического линейного представления групшы $G$ в пространстве $U$ и функцией $(\operatorname{det} g)^{-1}$. (Впрочем, функцию $(\operatorname{det} g)^{-1}$ можно иметь уже в числе матричных элементов, если добавить к исходному представлению одномерное представление $g \mapsto(\operatorname{det} g)^{-1}$.) По теореме Вейерштрасса о приближении непрерывных функций многочленами пространство $\mathbb{R}[G]$ плотно в $C(G)$.

Конечномерное линейное представление $R: G \rightarrow \mathrm{GL}(V)$ назовем полиномиальнblм, если его матричные элементы принадлежат алгебре $\mathbb{R}[G]$. Матричные элементы полиномиальных линейных представлений линейно порождают пространство $\mathbb{R}[G]$. В самом деле, группа $G$ действует в пространстве $\mathbb{R}[G]$ левьми сдвигами:

$$
(g \varphi)(x)=\varphi\left(g^{-1} x\right) .
$$

Так как степень полиномиальной функции (относительно матричных элементов тавтологического представления и функции $\left.(\operatorname{det} g)^{-1}\right)$ при этом сохраняется, то каждая функция $\varphi \in \mathbb{R}[G]$ порождает конечномерное инвариантное подпространство. Обозначим его через $W(\varphi)$. Пусть $\left\{\varphi_{1}, \ldots, \varphi_{m}\right\}$ - его базис и $x_{1}, \ldots, x_{m} \in G$ - такие точки, что $\varphi_{i}\left(x_{j}\right)=\delta_{i j}$. Имеем:

$$
\varphi_{i}(g x)=\sum_{j} a_{i j}(g) \varphi_{j}(x) .
$$

Подставляя $x=x_{k}$, получаем:

$$
a_{i k}(g)=\varphi_{i}\left(g x_{k}\right),
$$


откуда следует, что $a_{i k} \in \mathbb{R}[G]$. Но $a_{i k}$ - это матричные элементы представления групшы $G$ в пространстве $W(\varphi)^{*}=V(\varphi)$, сопряженном к $W(\varphi)$ (для которого $\varphi_{1}, \ldots, \varphi_{m}$ служат координатными функциями), так что представление $G: V(\varphi)$ полиномиально. С другой стороны, полагая в (13) $x=e$, получаем, что $\varphi_{1}, \ldots, \varphi_{m}$ принадлежат линейной оболочке матричных элементов этого представления.

Для любого линейного представления $R: G \rightarrow \mathrm{GL}(V)$ обозначим через $A(R)$ линейную оболочку операторов представления. Это подалгебра (ассоциативной) алгебры $\mathrm{L}(V)$. Обозначим через $A(R)^{K \times K}$ ее подалгебру, состоящую из линейных операторов, инвариантных относительно умножений слева и справа на операторы $R(k)$, $k \in K$. Она может рассматриваться как некоторая алгебра линейных операторов в пространстве

$$
V^{K}=\{v \in V: R(k) v=v \forall k \in K\} .
$$

ПРЕДЛОЖЕНИЕ 6. Пара $(G, K)$ является парой Гельфанда тогда и только тогда, когда для любого полиномиального линейного представления $R$ группь $G$ алгебра $A(R)^{K \times K}$ коммутативна.

ДокаЗАТЕЛЬСтво. Любое полиномиальное линейное представление $R: G \rightarrow$ $\mathrm{GL}(V)$ продолжается до представления групповой алгебры $\mathbb{R} G$. Образом алгебры $\mathbb{R} G$ при этом представлении является алгебра $A(R)$, а образом подалгебры $(\mathbb{R} G)^{K \times K}-$ подалгебра $A(R)^{K \times K}$. Так как пространство $\mathbb{R}[G]$ плотно в $C(G)$, то пересечение ядер всех получаемых таким образом линейных представлений алгебры $\mathbb{R} G$ равно нулю. Следовательно, коммутативность алгебры $(\mathbb{R} G)^{K \times K}$ равносильна коммутативности всех алгебр $A(R)^{K \times K}$.

Всякое полиномиальное линейное представление групшы $G$ (однозначно) продолжается до линейного представления алгебраической групшы $G^{a}$, причем линейные оболочки операторов представления для груп $G$ и $G^{a}$ одинаковы. Поэтому справедливо

СлеДСТВИЕ. Пара $(G, K)$ является парой Гельфанда тогда и только тогда, когда таковой является пара $\left(G^{a}, K\right)$.

5. Понятие пары Гельфанда может быть интерпретировано также в терминах инвариантных дифференциальных операторов на однородном пространстве $X=G / K$.

Обозначим через $\mathscr{D}(X)$ алгебру всех дифференциальных операторов на $X$ и через $\mathscr{D}(X)^{G}$ - подалгебру инвариантных дифференщиальных операторов.

Алгебра $\mathscr{D}(X)$ фильтрована по порядку дифференциального оператора. Соответствующая градуированная алгебра gr $\mathscr{D}(X)$ есть алгебра $\mathscr{P}\left(T^{*} X\right)$ функций на кокасательном расслоении $T^{*} X$ многообразия $X$, полиномиальных на слоях, причем изоморфизм осушествляется взятием символа дифференщиального оператора. Градуированная алгебра, соответствующая подалгебре $\mathscr{D}(X)^{G} \subset \mathscr{D}(X)$, вложена в подалгебру $\mathscr{P}\left(T^{*} X\right)^{G} \subset \mathscr{P}\left(T^{*} X\right)$ инвариантных функций на $T^{*} X$. Мы покажем, что эти алгебры на самом деле совпадают, и одновременно получим описание алгебры $\mathscr{D}(X)^{G}$ в терминах обертьвающей алгебры $U(\mathfrak{g})$ алгебры Ли $\mathfrak{g}$.

Каждому элементу $\xi$ алгебры Ли $\mathfrak{g}=T_{e}(G)$ поставим в соответствие левоинвариантный дифференциальный оператор первого порядка на групе $G$, состояший в дифференщировании по направлению левоинвариантного векторного поля, равного $\xi$ в единице групшы $G$. Это отображение продолжается до изоморфизма алгебры $U(\mathfrak{g})$ на 
алгебру левоинвариантных дифференциальных операторов на $G$. При этом порядок дифференщиального оператора, соответствующего элементу $u \in U(\mathfrak{g})$, равен степени выражения элемента $u$ через элементы алгебры Ли $\mathfrak{g}$, а взятие символа левоинвариантного дифференциального оператора в точке $e$ есть не что иное, как изоморфизм $\operatorname{gr} U(\mathfrak{g}) \rightarrow S(\mathfrak{g})$, описьваемьй теоремой Пуанкаре-Биркгофа-Витта.

Обозначим через $U(\mathfrak{g})^{K}$ подалгебру алгебры $U(\mathfrak{g})$, состояшую из элементов, инвариантных относительно присоединенного действия подгруппы $K$. Всякий оператор $D \in U(\mathfrak{g})^{K}$ перестановочен с правьми сдвигами на элементы подгруппы $K$ и, следовательно, переводит в себя пространство функций на $G$, правоинвариантных относительно $K$. Тем самым он определяет некоторьй инвариантньй дифференциальньй оператор $D_{K}$ на многообразии $X=G / K$. Отметим, что символ оператора $D_{K}$ есть образ символа оператора $D$ при каноническом гомоморфизме $\mathscr{P}\left(T^{*} G\right)^{G} \rightarrow \mathscr{P}\left(T^{*} X\right)^{G}$. В частности, символ оператора $D_{K}$ в точке $o \in X$ есть образ символа оператора $D$ в точке $e \in G$ при каноническом гомоморфизме $S(\mathfrak{g}) \rightarrow S(\mathfrak{g} / \mathfrak{k})$.

Все дифференциальные операторы из левого идеала $U(\mathfrak{g}) \mathfrak{k}$ алгебры $U(\mathfrak{g})$ аннулируют функции, правоинвариантные относительно $K$. Так как элементы подалгебры $U(\mathfrak{g})^{K}$ перестановочны с элементами из $\mathfrak{k}$, то $(U(\mathfrak{g}) \mathfrak{k})^{K}-$ двусторонний идеал алгебры $U(\mathfrak{g})^{K}$. Таким образом, определен гомоморфизм

$$
\gamma: U(\mathfrak{g})^{K} /\left(U(\mathfrak{g})^{\mathfrak{k}}\right)^{K} \rightarrow \mathscr{D}(X)^{G}
$$

ПРЕДЛОЖЕНИЕ 7 [16]. Гомоморфизм (14) является изоморфизмом. Градуированная алгебра, ассоциированная с $\mathscr{D}(X)^{G}$, совпадает с $\mathscr{P}\left(T^{*} X\right)^{G}$.

ДокАЗАТЕльство. Достаточно доказать, что композиция соответствующего гомоморфизма градуированных алгебр

$$
\operatorname{gr} \gamma: \operatorname{gr}\left(U(\mathfrak{g})^{K} /(U(\mathfrak{g}) \mathfrak{k})^{K}\right) \rightarrow \operatorname{gr} \mathscr{D}(X)^{G}
$$

вложения

$$
\operatorname{gr} \mathscr{D}(X)^{G} \hookrightarrow \mathscr{P}\left(T^{*} X\right)^{G}
$$

и изоморфизма ограничения

$$
\mathscr{P}\left(T^{*} X\right)^{G \stackrel{\sim}{\rightarrow}} \mathbb{R}\left[T_{o}^{*} X\right]^{K}=S(\mathfrak{g} / \mathfrak{k})^{K}
$$

является изоморфизмом. Указанная композищия есть не что иное, как взятие символа в точке $о$ дифференциального оператора на $X$, определенного элементом алгебры $U(\mathfrak{g})^{K} /(U(\mathfrak{g}) \mathfrak{k})^{K}$.

Из полной приводимости линейных представлений группы $K$ следует, что факторалгебра $U(\mathfrak{g})^{K} /(U(\mathfrak{g}) \mathfrak{k})^{K}$ как фильтрованное векторное пространство совпадает с $(U(\mathfrak{g}) / U(\mathfrak{g}) \mathfrak{k})^{K}$ и что соответствующее градуированное векторное пространство совпадает с $(\operatorname{gr}(U(\mathfrak{g}) / U(\mathfrak{g}) \mathfrak{k}))^{K}$. В силу теоремы Пуанкаре-Биркгофа-Витта пространство $\operatorname{gr}(U(\mathfrak{g}) / U(\mathfrak{g}) \mathfrak{k})$ канонически изоморфно $S(\mathfrak{g} / \mathfrak{k})$. Следовательно, подпространство его $K$-инвариантных элементов канонически изоморффн $S(\mathfrak{g} / \mathfrak{k})^{K}$.

ОПРЕДЕЛЕНИЕ 4. Однородное пространство $X=G / K$ называется коммутативнылм, если алгебра $\mathscr{D}(X)^{G}$ коммутативна. 
Tеорема 1 [16], [17]. Однородное пространство $X=G / K$ коммутативно тогда и только тогда, когда $(G, K)$ - пара Гельфанда.

ДокАЗАТЕльство. Для любого $\xi \in \mathfrak{g}$ рассмотрим формальньй левоинвариантньй дифференциальный оператор

$$
\operatorname{Exp} t \xi=\sum_{n=0}^{\infty} \frac{t^{n} \xi^{n}}{n !}
$$

на групше $G$. Для аналитической функции $\varphi$ справедливо равенство

$$
((\operatorname{Exp} t \xi) \varphi)(x)=\varphi(x(\exp t \xi))
$$

в том смысле, что для любого $x \in G$ и любого достаточно близкого к нулю $t \in \mathbb{R}$ ряд, стоящий в левой части, сходится и его сумма равна правой части.

Далее, для любого элемента $u \in U(\mathfrak{g})$ обозначим через $u^{\natural}$ результат его усреднения по присоединенному действию группы $K$ и положим

$$
(\operatorname{Exp} t \xi)^{\natural}=\sum_{n=0}^{\infty} \frac{t^{n}\left(\xi^{n}\right)^{\natural}}{n !} .
$$

Для аналитической функции $\varphi$, правоинвариантной относительно $K$, в том же смысле, что и вьше, справедливо равенство

$$
\left((\operatorname{Exp} t \xi)^{\natural} \varphi\right)(x)=\int_{K} \varphi(x k(\exp t \xi)) d k .
$$

Следовательно, для любых $\xi, \eta \in \mathfrak{g}$

$$
\left((\operatorname{Exp} t \xi)^{\natural}(\operatorname{Exp} s \eta)^{\natural} \varphi\right)(x)=\int_{K} \varphi_{x}((\exp t \xi) k(\exp s \eta)) d k
$$

где

$$
\varphi_{x}(y)=\int_{K} \varphi(x k y) d k .
$$

Отметим, что функция $\varphi_{x}$ двусторонне инвариантна относительно $K$.

В частности, если функция $\varphi$ двусторонне инвариантна относительно $K$, то, полагая в (15) $x=e$, получаем следуюшее выражение для интеграла (12) при $g=\exp t \xi$, $h=\exp s \eta$ :

$$
I(\varphi ; g, h)=\left((\operatorname{Exp} t \xi)^{\natural}(\operatorname{Exp} s \eta)^{\natural} \varphi\right)(e) .
$$

Если пространство $X$ коммутативно, то операторы $(\operatorname{Exp} t \xi)^{\natural}$ и $(\operatorname{Exp} s \eta)^{\natural}$ коммутируют на $X$ и, следовательно, $I(\varphi ; g, h)=I(\varphi ; h, g)$, т.е. $(G, K)$ - пара Гельфанда.

Обратно, записывая равенство (15) в виде

$$
\left((\operatorname{Exp} t \xi)^{\natural}(\operatorname{Exp} s \eta)^{\natural} \varphi\right)(x)=I\left(\varphi_{x} ; \exp t \xi, \exp s \eta\right),
$$

мы находим, что если $(G, K)$ - пара Гельфанда, то операторы $(\operatorname{Exp} t \xi)^{\natural}$ и $(\operatorname{Exp} s \eta)^{\natural}$ коммутируют на $X$ при любых $t$ и $s$, а это означает, что операторы $\left(\xi^{n}\right)^{\natural}$ и $\left(\eta^{m}\right)^{\natural}$ коммутируют на $X$ при любых $n$ и $m$. Так как степени элементов из $\mathfrak{g}$ линейно порождают 
алгебру $U(\mathfrak{g})$, то их усреднения по $K$ линейно порождают подалгебру $U(\mathfrak{g})^{K}$. Следовательно, все операторы из $U(\mathfrak{g})^{K}$ коммутируют на $X$, т.е. пространство $X$ коммутативно.

Дадим альтернативное доказательство теоремы в случае, когда $G \subset \mathrm{GL}(U)$ - линейная группа Ли.

Так как струи полиномиальных функций в любой точке могут быть произвольны, то при исследовании коммутативности дифференциальных операторов мы можем ограничиться рассмотрением их действия на полиномиальные функции. Далее, всякая полиномиальная функция при подходящем выборе полиномиального линейного представления может быть сделана линейной, т.е. линейной комбинацией матричных элементов этого представления. Посмотрим, как действуют инвариантные дифференциальные операторы на линейные функции.

Пусть $R: G \rightarrow \mathrm{GL}(V)$ - полиномиальное линейное представление групшы $G$ и $\rho=$ $d R: \mathfrak{g} \rightarrow \mathfrak{g l}(V)$ - соответствуюшее линейное представление ее касательной алгебры Ли $\mathfrak{g}$. Представление $\rho$ продолжается до представления обертьвающей алгебры $U(\mathfrak{g})$, которое мы будем обозначать той же буквой. Подалгебра $\rho(U(\mathfrak{g})) \subset \mathrm{L}(V)$ совпадает с определенной выше подалгеброй $A(R)$. Каждая линейная функция $\alpha$ на $A(R)$ определяет полиномиальную функцию $\widehat{\alpha}=\alpha \circ R$ на групе $G$. Получаемые таким образом функции на $G$ мы будем называть $R$-линейными.

Производная $R$-линейной функции $\widehat{\alpha}(g)=\alpha(R(g))$ по направлению левоинвариантного векторного поля $g \mapsto g \xi$ равна $\alpha(R(g) \rho(\xi))$; в частности, это тоже $R$-линейная функция. Отсюда следует, что результат применения к функции $\widehat{\alpha}(g)$ левоинвариантного дифференциального оператора, определяемого любым элементом $u \in U(\mathfrak{g})$, есть $\alpha(R(g) \rho(u))$.

Таким образом, действие левоинвариантных дифференциальных операторов в пространстве $R$-линейных функций описывается естественным линейным представлением $T$ алгебры $A(R)$ в сопряженном пространстве $A(R)^{*}$, определяемым формулой

$$
(T(a) \alpha)(x)=\alpha(x a) \quad\left(a, x \in A(R), \alpha \in A(R)^{*}\right) .
$$

Нас, однако, интересуют не все левоинвариантные дифференциальные операторы на $G$, а только правоинвариантные относительно $K$, т.е. определяемые элементами подалгебры $U(\mathfrak{g})^{K} \subset U(\mathfrak{g})$, и не все $R$-линейные функции, а только правоинвариантные относительно $K$, т.е. происходяшие из таких же линейных функций на $A(R)$. Подалгебра $U(\mathfrak{g})^{K}$ при представлении $\rho$ отображается на подалгебру

$$
A(R)^{K}=\{a \in A(R): a R(k)=R(k) a \forall k \in K\} \subset A(R) .
$$

Для любого $a \in A(R)^{K}$ положим

$$
a^{\natural}=\int_{K} a R(k) d k \in A(R)^{K \times K} .
$$

Очевидно, что оператор $T\left(a^{\natural}\right)$ действует в подпространстве $K$-инвариантных линейных функций так же, как $T(a)$; с другой стороны, он аннулирует дополнительное $K$-инвариантное подпространство. Поэтому коммутативность операторов из $U(\mathfrak{g})^{K}$ на пространстве $K$-инвариантных $R$-линейных функций равносильна коммутативности алгебры $A(R)^{K \times K}$.

Ввиду предложения 6 мы можем теперь заключить, что коммутативность однородного пространства $X=G / K$ равносильна тому, что $(G, K)$ - пара Гельфанда. 
СлЕДСТВИЕ. Всякое слабое симметрическое однородное пространство коммутативно.

Этот факт был доказан (другим способом) А. Сельбергом в [4]. Обратное утверждение неверно. Примеры коммутативных однородных пространств, не являющихся слабо симметрическими, будут приведены в $\S 4$ главы II настоящей статьи. Первые примеры такого рода были построены в [18].

\section{§3. Сферические однородные пространства редуктивных групп}

1. Линейная групша Ли $G \subset \mathrm{GL}(U)$, имеюшая конечное число связных компонент, называется редуктивной, если она вполне приводима или, что эквивалентно [19], если ее касательная алгебра Ли g есть прямая сумма полупростой алгебры Ли (совпадающей с коммутантом $[\mathfrak{g}, \mathfrak{g}]$ алгебры $\mathfrak{g})$ и коммутативной алгебры Ли, состояшей из полупростых линейных операторов (совпадающей с центром $\mathfrak{z}(\mathfrak{g})$ алгебры $\mathfrak{g})$.

В этом определении пространство $U$ и группа $G$ могут быть вешественными или комплексными. Для наших ближайших целей мы будем иметь в виду вешественный вариант.

Назовем алгеброй (комплексных ) полиномиальных функиий на $G$ и обозначим через $\mathbb{C}[G]$ алгебру комплексных функций на группе $G$, порожденную матричньми элементами ее тавтологического линейного представления в пространстве $U$ и функцией $(\operatorname{det} g)^{-1}$. (Подчеркнем, что $U$-вешественное векторное пространство.) Конечномерное комплексное линейное представление $R: G \rightarrow \mathrm{GL}(V)$ назовем полиномиальны.м, если его матричные элементы принадлежат алгебре $\mathbb{C}[G]$. Известно [19], что всякое полиномиальное линейное представление редуктивной групшы вполне приводимо.

Пусть $K \subset G$ - компактная подгруппа и $X=G / K$. Алгебру

$$
\mathbb{C}[X]=\mathbb{C}[G]^{K}=\{\varphi \in \mathbb{C}[G]: \varphi(g k)=\varphi(g) \forall g \in G, k \in K\}
$$

будем назьвать алгеброй (комплексных) полиномиальных функиий на однородном пространстве $X$. Так как орбиты компактной линейной группы разделяются полиномиальными инвариантами $[1]$, то алгебра $\mathbb{C}[X]$ разделяет точки многообразия $X$. Естественное линейное представление групш $G$ в пространстве $\mathbb{C}[X]$ разлагается в сумму неприводимых конечномерных представлений, сопряженных полиномиальньм представлениям (ср. рассуждение в $\S 2)$.

Для однородных пространств редуктивных групп свойство коммутативности особенно просто интерпретируется в терминах конечномерных линейных представлений.

ОПРЕДЕЛЕНИЕ 5. Однородное пространство $X=G / K$ редуктивной группы $G$ называется сферическим, если представление групш $G$ в пространстве $\mathbb{C}[X]$ имеет простой спектр (т.е. его неприводимые компоненты попарно не изоморфны).

Следующее предложение есть частньй случай двойственности Фробениуса.

ПРЕДЛОЖЕНИЕ 8. Однородное пространство $X=G / K$ редуктивной групnы $G$ является сферическим тогда и только тогда, когда для любого неприводимого полиномиального комплексного линейного представления $R: G \rightarrow \mathrm{GL}(V)$ подпространство $V^{K} \subset V$ не более чем одномерно. 
ДокАЗАТЕльство. Каждый вектор $v \in V^{K}$ порождает $G$-эквивариантное линейное отображение

$$
V^{*} \rightarrow \mathbb{C}[X], \quad \alpha \mapsto(g K \mapsto \alpha(R(g) v))
$$

и, обратно, всякое $G$-эквивариантное линейное отображение из $V^{*}$ в $\mathbb{C}[X]$ получается таким образом. Следовательно, кратность вхождения $R^{*}$ в представление $G: \mathbb{C}[X]$ равна $\operatorname{dim} V^{K}$.

ПРЕДЛОЖЕНИЕ 9. Однородное пространство $X=G / K$ редуктивной группьг $G$ является сферическим тогда и только тогда, когда оно коммутативно.

ДокАЗАТЕльСТВо. Для любого полиномиального комплексного линейного представления $R: G \rightarrow \mathrm{GL}(V)$ обозначим через $A(R)$ (комплексную) линейную оболочку операторов представления. Если $R$ неприводимо, то по теореме Бернсайда $A(R)=$ $\mathrm{L}(V)$. В обшем случае $A(R) \simeq A\left(R_{1}\right) \oplus \cdots \oplus A\left(R_{s}\right)$, где $R_{1}, \ldots, R_{s}$ - все (попарно не изоморфные) неприводимые представления, входящие в $R$.

Из теоремы 1 и предложения 6 следует, что пространство $X$ коммутативно тогда и только тогда, когда для любого полиномиального линейного представления $R: G \rightarrow$ $\mathrm{GL}(V)$ алгебра $A(R)^{K \times K}$ коммутативна. В силу предыдущего достаточно рассматривать только неприводимые представления; но если $R$ неприводимо, то

$$
A(R)^{K \times K}=\mathrm{L}(V)^{K \times K} \simeq \mathrm{L}\left(V^{K}\right),
$$

так что коммутативность алгебры $A(R)^{K \times K}$ равносильна тому, что $\operatorname{dim} V^{K} \leqslant 1$.

СлЕДСТВИЕ. Всякое слабо симметрическое (и, тем более, всякое симметрическое) однородное пространство редуктивной группы является сферическим.

Как уже упоминалось в $\S 2$, сферичность компактных симметрических пространств была доказана Э. Картаном и, затем, более обшим методом, И. М. Гельфандом. В простейшем случае двумерной сферы $S^{2}=\mathrm{SO}_{3} / \mathrm{SO}_{2}$ она была известна много раншше благодаря сферическим функциям Лапласа, применявшимся в математической физике. Отсюда и происходит термин “сферические однородные пространства”.

2. В теории алгебраических групп и теории инвариантов сферические однородные пространства обычно трактуются несколько иначе. Пусть редуктивная комплексная алгебраическая групша $G$ действует на неприводимом аффинном комплексном алгебраическом многообразии $X$. Действие $G: X$ назьвается сферическим, если естественное линейное представление $G: \mathbb{C}[X]$ имеет простой спектр. В случае, когда $X=G / H$, где $H$ - редуктивная алгебраическая подгруппа групшы $G$, это свойство эквивалентно тому, что $\operatorname{dim} V^{H} \leqslant 1$ для любого неприводимого линейного представления $R: G \rightarrow \mathrm{GL}(V)$. Если это выполнено, то однородное пространство $G / H$, a также подгруппа $H$ называются сферическими.

Покажем, как связаны между собой эти два подхода. Пусть $G \subset \mathrm{GL}(U)-$ редуктивная вещественная линейная группа Ли, $K$ - ее компактная подгруппа и $X=G / K$. Обозначим через $G^{a}(\mathbb{C})$ замыкание по Зарисскому групшы $G$ в группе $\mathrm{GL}(U(\mathbb{C}))$, где $U(\mathbb{C})$ - комплексификация пространства $U$. Это редуктивная алгебраическая группа; ее касательная алгебра $\mathfrak{g}^{a}(\mathbb{C})$ содержит комплексификацию $\mathfrak{g}(\mathbb{C})$ алгебры $\mathfrak{g}$ и имеет такой же коммутант, что и $\mathfrak{g}(\mathbb{C})[1]$. Структурная алгебра $\mathbb{C}\left[G^{a}(\mathbb{C})\right]$ группы $G^{a}(\mathbb{C})$ 
путем ограничения функций изоморфна алгебре $\mathbb{C}[G]$. Условимся отождествлять эти алгебры. Пусть, далее, $K(\mathbb{C})$ - замькание по Зарисскому группы $K$ в $\operatorname{GL}(U(\mathbb{C}))$. Это редуктивная алгебраическая подгруппа групшы $G^{a}(\mathbb{C})$. Так как группа $K$ алгебраична $[1]$, то касательная алгебра группы $K(\mathbb{C})$ совпадает с комплексификацией $\mathfrak{k}(\mathbb{C})$ алгебры k.

Рассмотрим (аффинное) алгебраическое многообразие $X^{a}(\mathbb{C})=G^{a}(\mathbb{C}) / K(\mathbb{C})$. Его структурная алгебра $\mathbb{C}\left[X^{a}(\mathbb{C})\right]=\mathbb{C}\left[G^{a}(\mathbb{C})\right]^{K(\mathbb{C})}$ совпадает с алгеброй $\mathbb{C}[X]=\mathbb{C}[G]^{K}$, и представления груп $G$ и $G^{a}(\mathbb{C})$ в этой алгебре имеют одни и те же инвариантные подпространства. Кроме того, если многообразие $X$ связно, то алгебраическое многообразие $X^{a}(\mathbb{C})$ неприводимо. Следовательно, однородное пространство $X=G / K$ является сферическим в смысле определения 5 тогда и только тогда, когда однородное пространство $X^{a}(\mathbb{C})=G^{a}(\mathbb{C}) / K(\mathbb{C})$ является сферическим в смысле теории алгебраических групп.

3. В оставшейся части этого параграфра мы будем говорить в основном о сферических однородных пространствах в смысле теории алгебраических групп.

Имеется следующий геометрический критерий сферичности.

Теорема 2 [20]. Действие редуктивной алгебраической группы $G$ на неприводимом аффинном многообразии $X$ является сферическим тогда и только тогда, когда борелевская подгруппа $B$ группы $G$ имеет в $X$ открытую орбиту.

ДокАЗАТЕЛЬСтво. Пусть многообразие $X$ не сферично, ипусть $W_{1}$ и $W_{2}-$ два минимальных инвариантных подпространства алгебры $\mathbb{C}[X]$, изоморфных как $G$-модули. Тогда $W_{1}$ и $W_{2}$ изоморфны и как $G^{0}$-модули и, следовательно, содержат непропорциональные функции, являющиеся весовыми векторами для борелевской подгруппы $B$ с одним и тем же весом. Отношение этих функций является $B$-инвариантной рациональной функцией, которая не позволяет групе $B$ иметь открытую орбиту в $X$.

Обратно, пусть группа $B$ не имеет открытой орбиты в $X$. Тогда по теореме Розенлихта [2] сушествует не постоянная $B$-инвариантная рациональная функция $\varphi$ на $X$. Представим ее в виде отношения полиномиальных функций: $\varphi=\varphi_{1} / \varphi_{2}, \varphi_{1}, \varphi_{2} \in$ $\mathbb{C}[X]$. Наименьшее $B$-инвариантное подпространство, содержашее $\varphi_{1}$, конечномерно, и в нем должен быть весовой вектор для $B$. Пусть это будет $\sum_{i} c_{i} b_{i} \varphi_{1}\left(c_{i} \in \mathbb{C}, b_{i} \in B\right)$. Так как $\varphi_{1} / \varphi_{2}=b_{i} \varphi_{1} / b_{i} \varphi_{2}$ при всех $i$, то

$$
\varphi=\frac{\sum_{i} c_{i} b_{i} \varphi_{1}}{\sum_{i} c_{i} b_{i} \varphi_{2}} .
$$

Знаменатель этой дроби автоматически является весовьм вектором для $B$, имеющим тот же вес, что и числитель. Таким образом, можно считать, что $\varphi_{1}$ и $\varphi_{2}-$ весовые векторы одного веса для подгруппы $B$; но тогда порождаемые ими $G^{0}$-инвариантные подпространства изоморфны как $G^{0}$-модули. Это уже доказывает, что многообразие $X$ не сфрерично относительно группы $G^{0}$.

Для того чтобы доказать, что многообразие $X$ не сферично относительно всей группы $G$ (если последняя не связна), заметим, что для любого $n$ функции

$$
\varphi_{1}^{n}, \varphi_{1}^{n-1} \varphi_{2}, \ldots, \varphi_{1} \varphi_{2}^{n-1}, \varphi_{2}^{n}
$$

также являются весовыми векторами одного веса для борелевской подгрупшы $B$. Докажем, что они линейно независимы. В самом деле, пусть

$$
a_{0} \varphi_{1}^{n}+a_{1} \varphi_{1}^{n-1} \varphi_{2}+\cdots+a_{n-1} \varphi_{1} \varphi_{2}^{n-1}+a_{n} \varphi^{n}=0
$$


- нетривиальная линейная зависимость. Можно считать, что $a_{0} \neq 0$. Пусть $\lambda-$ какой-нибудь корень многочлена $a_{0} x^{n}+a_{1} x^{n-1}+\cdots+a_{n-1} x+a_{n}$. Тогда из отсутствия делителей нуля в $\mathbb{C}[X]$ следует, что $\varphi_{1}-\lambda \varphi_{2}=0$, но это противоречит тому, что функция $\varphi$ не постоянна.

Из этого следует, что кратности неприводимых представлений группы $G^{0}$ в представлении $G^{0}: \mathbb{C}[X]$ не ограничены. С другой стороны, число неприводимых представлений групшы $G$, содержаших данное неприводимое представление $R_{0}$ групшы $G^{0}$, не превосходит индекса $\left[G: G^{0}\right]$, так как все они являются неприводимьми компонентами представления групшы $G$, индуцированного представлением $R_{0}$. С ледовательно, среди неприводимых компонент представления $G: \mathbb{C}[X]$ должны быть изоморфные.

Наличие открытой орбиты борелевской подгрупшы в $X$ равносильно тому, что сама групша $G$ имеет в $X$ открытую орбиту и если $H$ - стабилизатор подходящей точки этой орбиты, то

$$
\mathfrak{g}=\mathfrak{h}+\mathfrak{b}
$$

СлЕДСТВИЕ 1. Свойство однородного пространства $G / H$ быть сферическим является локальнылм, т.е. зависит только от алгебры Ли $\mathfrak{g}$ и ее подалгебры $\mathfrak{h}$.

СлЕДСТВИЕ 2. Однородное пространство $G / H$ связной редуктивной групnы $G$ является сферическим тогда и только тогда, когда таковым является однородное пространство $(G / Z) / H$, где $Z$ - чентр группь $G$.

Таким образом, описание сфферических однородных пространств сводится к случаю, когда группа $G$ связна и полупроста.

СЛЕДСТВИЕ 3. Если однородное пространство $G / H$ является сферическим и $F \subset G-$ - едуктивная подгруппа, содержащая $H$, то однородное пространство F/H также является сферическим.

4. Пусть $N(H)$ (соответственно $Z(H)$ ) обозначает нормализатор (соответственно централизатор) подгруппы $H$ в группе $G$. Известно, что если група $H$ редуктивна, то групшы $N(H)$ и $Z(H)$ также редуктивны, причем $N(H)$ является конечным расширением подг руппы $H Z(H)$.

ПРЕДЛОЖЕНИЕ 10. Если $X=G / H$ - сферическое однородное пространство, то әруппа $N(H) / H$ абелева (и, значит, является прямым произведением (алгебраического) тора и конечной абелевой группь).

(Ср. предложение 2.)

ДоказАтельство. Группа $N(H) / H$ действует на $G / H=X$ правыми умножениями. Это действие перестановочно с действием групш $G$. Рассмотрим возникаюшее при этом линейное представление групшы $N(H) / H$ в $\mathbb{C}[X]$. Так как представление $G: \mathbb{C}[X]$ имеет простой спектр, то группа $N(H) / H$ обязана переводить каждую его неприводимую компоненту в себя и действовать на ней скалярно. Следовательно, эта группа абелева.

Предположим, что групшы $G$ и $H$ связны. При этом условии назовем сферическое пространство $G / H$ насыщенным, а сферическую подгруппу $H \subset G$ - насыщенной 
подгруппой, если группа $N(H) / H$ конечна. Легко видеть, что если $G / H$ - любое сферическое пространство, то $G / N(H)^{0}$ - насьшенное сферическое пространство; назовем его насыщением пространства $G / H$. Из предложения 10 следует, что подгруппа $H$ является (почти прямым) произведением коммутанта группы $N(H)^{0}$ и некоторого подтора ее центра.

Далее, если группа $G$ проста, то ее присоединенное представление неприводимо и, значит, для любой сферической подгруппы $H$ имеет место неравенство $\operatorname{dim} \mathfrak{g}^{H} \leqslant 1$; в частности, центр подгрупшы $H$ не более чем одномерен.

5. К числу сферических однородных пространств относятся все комплексные симметрические однородные пространства редуктивных групп.

Пусть $G$ - комплексная алгебраическая група и $H$ - ее редуктивная алгебраическая подгруппа. Тогда однородное пространство $X=G / H$ является аффинным алгебраическим многообразием [2]. Предположим, что оно неприводимо; тогда оно называется (комплексным) симметрическим пространством, а подгруппа $H$ - симметрической подгруппой группы $G$, если сушествует такой инволютивньй автоморфизм $\sigma$ группы $G$, что $\mathfrak{h}=\mathfrak{g}^{\sigma}$. Комплексные симметрические пространства могут быть также определены и любым из способов, описанных в $\S 1$.

Комплексные симметрические однородные пространства редуктивных групп - это в точности комплексификации компактных римановых симметрических пространств. В частности, отсюда следует, что они являются сферическими.

6. Все сфрерические однородные пространства простых алгебраических групп были найдены М. Кремером [21], а сферические однородные пространства непростых полупростых алгебраических групп - И. В. Микитюком [22] и М. Брионом [23]. Приведем здесь результаты этой классификации.

В таблице 1 перечислены все, с точностью до локального изоморфизма, сферические однородные пространства простых комплексных алгебраических групп, не являющиеся локально симметрическими. Во всех случаях, когда подгруппа $H$ указана в виде $H_{1} \times H_{2}$, пространство тавтологического линейного представления (классической) групшы $G$ разлагается в прямую сумму двух подпространств, на одном из которых действует $H_{1}$, а на другом $-H_{2}$.

Сферические пространства с номерами 1, 3, 6, 11 таблицы 1 не являются насышенными. Насьшениями пространств с номерами 1, 6, 11 являются симметрические пространства; насышением пространства № 3 является пространство № 2 .

Очевидно, что прямое произведение

$$
\left(G_{1} / H_{1}\right) \times\left(G_{2} / H_{2}\right)=\left(G_{1} \times G_{2}\right) /\left(H_{1} \times H_{2}\right)
$$

сферических пространств $G_{1} / H_{1}$ и $G_{2} / H_{2}$ также является сферическим. Такие сферические пространства, а также локально изоморфные им назовем приводимыми. Сферическое пространство, насьшение которого неприводимо, назовем строго неприводимым.м.

В таблице 2 перечислены все, с точностью до локального изоморфизма, строго неприводимые сферические однородные пространства непростых полупростых комплексных алгебраических груп, не являюшиеся локально симметрическими. В последнем столбце изображена схема, описывающая вложение групшы $H$ в $G$. Ее белые 
ТАБЛИЦА 1

(Все группы - комплексные.)

\begin{tabular}{|c|c|c|}
\hline & $G$ & $H$ \\
\hline 1 & $\mathrm{SL}_{n}$ & $\mathrm{SL}_{m} \times \mathrm{SL}_{n-m}(m \neq n / 2)$ \\
\hline 2 & $\mathrm{SL}_{2 n+1}$ & $\mathbb{C}^{*} \mathrm{Sp}_{2 n}$ \\
\hline 3 & $\mathrm{SL}_{2 n+1}$ & $\mathrm{Sp}_{2 n}$ \\
\hline 4 & $\mathrm{Sp}_{n}$ & $\mathrm{Sp}_{n-2} \times \mathrm{SO}_{2}$ \\
\hline 5 & $\mathrm{SO}_{2 n+1}$ & $\mathrm{GL}_{n}$ \\
\hline 6 & $\mathrm{SO}_{4 n+2}$ & $\mathrm{SL}_{2 n+1}$ \\
\hline 7 & $\mathrm{SO}_{10}$ & $\mathrm{Spin}_{7} \times \mathrm{SO}_{2}$ \\
\hline 8 & $\mathrm{SO}_{9}$ & $\mathrm{Spin}_{7}$ \\
\hline 9 & $\mathrm{SO}_{8}$ & $\mathrm{G}_{2}$ \\
\hline 10 & $\mathrm{SO}_{7}$ & $\mathrm{G}_{2}$ \\
\hline 11 & $\mathrm{E}_{6}$ & $\mathrm{Spin}_{10}$ \\
\hline 12 & $\mathrm{G}_{2}$ & $\mathrm{SL}_{3}$ \\
\hline
\end{tabular}

ТАБЛИЦА 2

(Все группы - комплексные.)

\begin{tabular}{|c|c|c|c|}
\hline & $G$ & $H$ & Схема вложения \\
\hline 1 & $\mathrm{SL}_{n} \times \mathrm{SL}_{n+1}$ & $\mathrm{SL}_{n} \times \mathbb{C}^{*}$ & \\
\hline 2 & $\mathrm{SL}_{n} \times \mathrm{Sp}_{m}$ & $\mathrm{GL}_{n-2} \times \mathrm{SL}_{2} \times \mathrm{Sp}_{m-2}$ & \\
\hline 3 & $\mathrm{SL}_{n} \times \operatorname{Sp}_{m}(n \geqslant 5)$ & $\mathrm{SL}_{n-2} \times \mathrm{SL}_{2} \times \mathrm{Sp}_{m-2}$ & \\
\hline 4 & $\mathrm{Sp}_{n} \times \mathrm{Sp}_{m}$ & $\mathrm{Sp}_{n-2} \times \mathrm{Sp}_{2} \times \mathrm{Sp}_{m-2}$ & \\
\hline 5 & $\mathrm{Sp}_{n} \times \mathrm{Sp}_{4}$ & $\mathrm{Sp}_{n-4} \times \mathrm{Sp}_{4}$ & \\
\hline 6 & $\mathrm{SO}_{n} \times \mathrm{SO}_{n+1}$ & $\mathrm{SO}_{n}$ & \\
\hline 7 & $\mathrm{Sp}_{n} \times \mathrm{Sp}_{m} \times \mathrm{Sp}_{l}$ & $\mathrm{Sp}_{n-2} \times \mathrm{Sp}_{m-2} \times \mathrm{Sp}_{l-2} \times \mathrm{Sp}_{2}$ & \\
\hline 8 & $\mathrm{Sp}_{n} \times \mathrm{Sp}_{4} \times \mathrm{Sp}_{m}$ & $\mathrm{Sp}_{n-2} \times \mathrm{Sp}_{2} \times \mathrm{Sp}_{2} \times \mathrm{Sp}_{m-2}$ & \\
\hline
\end{tabular}


вершины отвечают множителям группы $G$, а черные - множителям группы $H$. Порядок вершин такой же, как порядок соответствующих им множителей, за исключением строки 8, где верхняя черная вершина отвечает последнему множителю группы $H$. Множитель группы $H$, соответствуюший черной вершине $v$, вкладывается диагональным образом в произведение тех множителей группы $G$, которые соответствуют белым вершинам, соединенньм с $v$. (Множитель вида $\operatorname{Sp}_{n-2}$ групшы $H$ при $n=2$ исчезает вместе с отвечаюшей ему черной вершиной.)

Все сферические пространства таблицы 2 , кроме пространства № 3 , являются насьшенными. Насышением пространства № 3 является пространство № 2 .

Заметим, что пример $3 \S 1$ есть первьй член серии сферических однородных пространств $\left(\mathrm{SO}_{n} \times \mathrm{SO}_{n+1}\right) / \mathrm{SO}_{n}$, получаемьй при $n=3$.

7. По аналогии с определением риманова слабо симметрического пространства можно дать определение комплексного слабо симметрического пространства. Возьмем, например, за основу свойство (WS1) из предложения 1.

Пусть $G$ - комплексная алгебраическая группа и $H$ - ее редуктивная алгебраическая подгруппа. Предположим, что (афффинное) однородное пространство $X=G / H$ неприводимо. Назовем его (комплексным) слабо симметрическим пространством, если сушествует такой автоморфизм $s$ многообразия $X$, что $s G s^{-1}=G$ и условие $(7)$ выполнено для всех пар $(x, y)$ "обшего положения", т.е. для всех пар открытого по Зарисскому подмножества в $X \times X$. Нетрудно доказать, что всякое комплексное симметрическое пространство является слабо симметрическим. (Однако это было бы неверно, если бы мы потребовали, чтобы условие (7) было вьполнено для всех $x, y$.)

Аналогично лемме 1 можно доказать, что эквивалентность относительно группы $G$ в условии (7) может быть заменена эквивалентностью относительно $G^{0}$. Отсюда, в частности, следует, что всякое слабо симметрическое однородное пространство группы $G$ является слабо симметрическим и относительно $G^{0}$.

Кроме того, очевидно, что если $X=G / H$ - слабо симметрическое пространство и $Z$-центр групшы $G$, то $X / Z=(G / Z) / H$ - также слабо симметрическое пространство.

Так же, как в доказательстве предложения 1 , устанавливается, что однородное пространство $X=G / H$ является слабо симметрическим тогда и только тогда, когда сушествует такой автоморфизм $\sigma$ группы $G$, что $\sigma(H)=H$ и

$$
\sigma(g) \in H g^{-1} H
$$

для всех $g$ из некоторого плотного открытого подмножества группы $G$.

8. С ледующее предложение является вариащией на тему предложений 4, 6 и 9.

ПРЕДЛОЖЕНИЕ 11. Всякое комплексное слабо симметрическое однородное пространство редуктивной алгебрачческой группы является сферическим.

ДокАЗАТЕльство. Заменив группу $G$ на ее связную компоненту и профакторизовав по центру, можно свести доказательство к случаю, когда группа $G$ связна и полупроста.

При этих предположениях пусть $\sigma$ - автоморфизм группы $G$, удовлетворяюший условию (17). Докажем, что для любого неприводимого представления $R: G \rightarrow \mathrm{GL}(V)$ алгебра $A(R)^{H \times H}$ коммутативна, откуда и будет следовать, что пространство $X=$ $G / H$ является сферическим (ср. доказательство предложения 9). 
Сушествует конечное расширение $\widehat{G}$ групшы $G$, в котором автоморфизм $\sigma$ является внутренним, и существует ортогональное представление $\widehat{S}: \widehat{G} \rightarrow \mathrm{GL}(W)$ групшы $\widehat{G}$, ограничение которого на $G$ содержит представление $R$. Обозначим это ограничение через $S$, и пусть $A(S)$ - линейная оболочка операторов из $S(G)$. Автоморфизм $\sigma$ индуцирует автоморфизм алгебры $A(S)^{H \times H}$, которьй ввиду (17) совпадает с транспонированием (относительно инвариантной симметрической билинейной формы). Следовательно, алгебра $A(S)^{H \times H}$ и, тем более, ее подалгебра $A(R)^{H \times H}$ коммутативны.

9. Имеет место обрашение предложения 11, причем даже в более точной форме. Для того чтобы его сформулировать, введем некоторые понятия.

Пусть $G$ - связная редуктивная комплексная алгебраическая група. Инволютивньй автоморфизм группы $G$ назьвается инволюиией Вейля, если он действует как инверсия на некотором максимальном торе группы $G$. Известно, что инволюции Вейля сушествуют, и все они сопряжены при помоши внутренних автоморфизмов.

Если $\theta$ - инволюция Вейля, то для любого линейного представления $R$ группы $G$ представление $R^{\theta}=R \circ \theta$ (в том же пространстве) изоморфно представлению $R^{*}$, сопряженному (= дуальному) $R$.

ПРЕДЛОЖЕНИЕ 12. Если инволючия Вейля $\theta$ группы $G$ сохраняет сферическую подгруппу $H$, то она действует на $N(H) / H$ как инверсия.

ДокАЗАТЕльство. Пусть $X=G / H$ и $\mathfrak{R}$ - множество неприводимых представлений группы $G$, входящих в разложение представления $G: \mathbb{C}[X]$. Для всякого $R \in \Re$ обозначим через $V(R)$ то единственное подпространство в $\mathbb{C}[X]$, на котором реализуется представление $R$. Тогда

$$
\mathbb{C}[X]=\bigoplus_{R \in \mathfrak{R}} V(R) .
$$

Группа $N(H) / H$ действует на каждом из подпространств $V(R)$ умножением на некоторьй характер $\chi_{R}$ (см. доказательство предложения 10).

Известно [24], что если $R \in \mathfrak{R}$, то и $R^{*} \in \mathfrak{R}$, причем $G$-инвариантное спаривание между $V(R)$ и $V\left(R^{*}\right)$ может быть определено как проекция произведения функций на компоненту разложения (18), отвечающую тривиальному представлению (т.е. на пространство констант). Отсюда следует, что это спаривание инвариантно также относительно $N(H) / H$ и, значит,

$$
\chi_{R^{*}}=\chi_{R}^{-1}
$$

Автоморфизм многообразия $X$, определяемьй инволюцией $\theta$, переставляет подпространства $V(R)$ и $V\left(R^{*}\right)$. Поэтому из (19) следует, что $\theta$ действует на $N(H) / H$ как инверсия.

10. Будем назьвать инволюцию Вейля группы $G$ согласованной с редуктивной подгруппой $H$, если она сохраняет $H$ и индуцирует на $H^{0}$ инволюцию Вейля.

Теорема 3 [25]. Пусть $X=G / H$ - сферическое однородное пространство связной редуктивной комплексной алгебраической группы $G$. Тогда

1) существует инволюция Вейля группь $G$, согласованная с $H$;

2) пространство $X$ является слабо симметрическим относительно любой инволючии Вейля группь $G$, сохраняющей $H$. 
ДокАЗАТЕльство. Благодаря предложению 12 доказательство первой части теоремы сводится к случаю, когда группа $H$ связна. Кроме того, путем несложных рассуждений, которые мы опустим, оно сводится к случаю, когда группа $G$ проста.

Рассмотрим случай, когда $H$ - связная симметрическая подгруппа, определяемая какой-то инволюцией $\sigma$ группы $G$. Существует $\sigma$-инвариантный максимальный тор $T$ групшы $G$, содержащий максимальньй тор подгруппы $H$, и $\sigma$-инвариантная борелевская подгруппа $B$, содержащая $T$ [26] (см. также [27]). Более того, можно выбрать канонические образующие $h_{i}, e_{i}, f_{i}(i=1, \ldots, n)$ алгебры Ли $\mathfrak{g}$, согласованные с $T$ и $B$, таким образом, что автоморфизм $\sigma$ будет действовать следуюшим образом:

$$
\begin{gathered}
\sigma\left(e_{i}\right)=e_{j}, \sigma\left(f_{i}\right)=f_{j}, \quad \text { если } \sigma\left(h_{i}\right)=h_{j}, i \neq j, \\
\sigma\left(e_{k}\right)=\varepsilon_{k} e_{k}, \sigma\left(f_{k}\right)=\varepsilon_{k} f_{k}\left(\varepsilon_{k}= \pm 1\right), \text { если } \sigma\left(h_{k}\right)=h_{k} .
\end{gathered}
$$

Тогда инволющия Вейля группы $G$, действующая как инверсия на $T$ и переводяшая $e_{i}$ в $-f_{i}$ (и $f_{i}$ в $-e_{i}$ ), коммутирует с $\sigma$ и, следовательно, сохраняет подгрупу $H$ (и индуцирует на ней инволюцию Вейля).

Остается рассмотреть несимметрические сферические подгруппы простых груп. K сожалению, доказательство сушествования требуемой инволющии Вейля в этом случае, приведенное в [25], хотя и несложно, но использует классификацию этих подгрупп (содержащуюся в таблице 1). Было бы интересно придумать более концептуальное доказательство.

Пусть теперь $\theta$ - любая инволюция Вейля группы $G$, сохраняюшая $H$, и $f$ - определяемьй ею автоморфизм многообразия $X$. Рассмотрим отображение

$$
F: X \times X \rightarrow X \times X, \quad(x, y) \mapsto(f y, f x)
$$

Это автоморфизм многообразия $X \times X$, нормализуюший диагональное действие группы $G$. Слабая симметричность пространства $X$ относительно $\theta$ означает, что $F$ переводит в себя $G$-орбиты общего положения. Мы покажем, что $F$ действует тривиально на алгебре $\mathbb{C}[X \times X]^{G} G$-инвариантных полиномиальных функций на $X \times X$ и, тем самым, на категорном факторе $(X \times X) / / G$, которьй по определению есть спектр этой алгебры. Так как категорный фактор разделяет замкнутые орбиты [2], а орбиты общего положения для действия $G$ на $X \times X$ замкнуты [24], то отсюда будет следовать, что пространство $X$ слабо симметрично относительно $\theta$.

В обозначениях доказательства предложения 12 имеем:

$$
\mathbb{C}[X \times X]=\mathbb{C}[X] \otimes \mathbb{C}[X]=\bigoplus_{R, S \in \mathfrak{R}}(V(R) \otimes V(S)),
$$

откуда

$$
\mathbb{C}[X \times X]^{G}=\bigoplus_{R \in \Re} \mathbb{C} \varepsilon_{R}
$$

где инвариант $\varepsilon_{R} \in V(R) \otimes V\left(R^{*}\right)$ строится по любым сопряженным базисам $\left\{e_{i}\right\}$ и $\left\{e_{i}^{*}\right\}$ пространств $V(R)$ и $V\left(R^{*}\right)$ по формуле

$$
\varepsilon_{R}=\sum_{i} e_{i} \otimes e_{i}^{*}
$$


Из данного в доказательстве предложения 12 описания спаривания между $V(R)$ и $V\left(R^{*}\right)$ ясно, что оно инвариантно относительно $f$ и, значит, $\left\{f e_{i}^{*}\right\}$ и $\left\{f e_{i}\right\}$ - также сопряженные базисы пространств $V(R)$ и $V\left(R^{*}\right)$. Следовательно,

$$
F \varepsilon_{R}=\sum_{i} f e_{i}^{*} \otimes f e_{i}=\varepsilon_{R}
$$

11. Выведем из доказанной теоремы ее вещественный аналог, что даст нам обращение следствия предложения 9.

Теорема 4 [25]. Всякое сферическое однородное пространство $X=G / K$ связной редуктивной вещественной группьл Ли $G$ слабо симметрично.

ДоКАЗАТЕЛЬСТВо. Без ограничения общности можно считать группу $G$ связной компонентой алгебраической групш. Рассмотрим комплексификацию $X(\mathbb{C})=$ $G(\mathbb{C}) / K(\mathbb{C})$ пространства $X$. Согласно теореме 3 пространство $X(\mathbb{C})$ является слабо симметрическим относительно любой инволюции Вейля группы $G(\mathbb{C})$, сохраняющей $K(\mathbb{C})$. Покажем, что эту инволюцию можно выбрать так, чтобы она сохраняла $G$.

Пусть $L$ - максимальная компактная подгруппа группы $G$, содержашая $K$, и $\sigma$-инволюция Картана относительно $L$, т.е. такой инволютивный автоморфизм группы $G$, что $L=G^{\sigma}$. Продолжим $\sigma$ до инволютивного автоморфизма группы $G(\mathbb{C})$. Сушествуют канонические образующие $h_{i}, e_{i}, f_{i}(i=1, \ldots, n)$ алгебры Ли $\mathfrak{g}(\mathbb{C})$, на которые $\sigma$ действует по формулам (20). Пусть $\theta$ - инволюция Вейля группы $G(\mathbb{C})$, действуюшая по формулам

$$
\theta\left(h_{i}\right)=-h_{i}, \quad \theta\left(e_{i}\right)=-f_{i}, \quad \theta\left(f_{i}\right)=-e_{i} .
$$

Тогда $\theta$ и $\sigma$ перестановочны и

$$
\mathfrak{g}=\{\xi \in \mathfrak{g}(\mathbb{C}): \theta \sigma(\xi)=\bar{\xi}\}
$$

где черта обозначает комплексное сопряжение относительно образующих $h_{i}, e_{i}, f_{i}$. Отсюда следует, что $\theta$ сохраняет $G$ и $L$. Более того, так как картановская подалгебра $\left\langle h_{1}, \ldots, h_{n}\right\rangle$ алгебры $\mathfrak{g}(\mathbb{C})$ содержит картановскую подалгебру алгебры $\mathfrak{l}$, то $\theta$ индуцирует на $L$ инволюцию Вейля. (Инволюции Вейля связных компактных групп Ли определяются аналогично инволюциям Вейля связных редуктивных комплексных алгебраических групп.) Заметим, что все указанные свойства инволюции $\theta$ сохраняются при ее сопряжении любым элементом из $L$.

Применим теперь теорему 3 к сферическому однородному пространству $L(\mathbb{C}) / K(\mathbb{C})$. Мы получим, в частности, что существует инволюция Вейля $\theta_{0}$ группы $L(\mathbb{C})$, сохраняюшая $K(\mathbb{C})$. Ее ограничение на $K(\mathbb{C})$ сохраняет некоторую компактную вешественную форму группы $K(\mathbb{C}) ;$ можно считать, что ею является $K$. Далее, инволюция $\theta_{0}$ должна сохранять некоторую компактную вешественную форму групшы $L(\mathbb{C})$, содержашую $K$; можно считать, что ею является $L$.

Так как все инволюции Вейля группы $L$ сопряжены, то можно считать, что $\left.\theta\right|_{L}=\left.\theta_{0}\right|_{L}$; тогда $\theta$ coхраняет $G$ и $K$.

По теореме 3 пространство $X(\mathbb{C})$ слабо симметрично относительно $\theta$. Это означает, что автоморфизм $F$ многообразия $X(\mathbb{C})$, определенньй по формуле $(21)$, действует на $(X(\mathbb{C}) \times X(\mathbb{C})) / / G(\mathbb{C})$ тривиально. Рассматривая $X(\mathbb{C}) \times X(\mathbb{C})$ как однородное 
расслоение над $X(\mathbb{C})=G(\mathbb{C}) / K(\mathbb{C})$ со слоем $X(\mathbb{C})$, мы получаем естественньй изоморфизм категорных факторов $(X(\mathbb{C}) \times X(\mathbb{C})) / / G(\mathbb{C})$ и $X(\mathbb{C}) / / K(\mathbb{C})$. Аналогично, имеется естественньй изоморфизм факторов $(X \times X) / G$ и $X / K$. Эти изоморфизмы включаются в коммутативную диаграмму

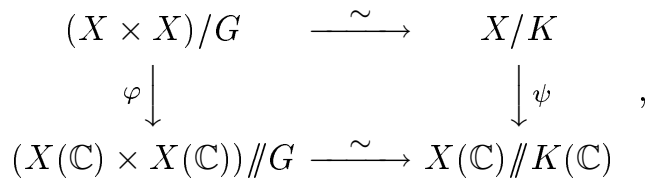

где $\varphi$ и $\psi$ индуцированы вложением $X \hookrightarrow X(\mathbb{C})$. При этом отображение $\varphi$ перестановочно с действием $F$.

Так как группа $K$ компактна, то ее орбиты в $X$ разделяются полиномиальньми инвариантами. Следовательно, отображение $\psi$, а значит, и отображение $\varphi$, инъективно. Но тогда $F$ действует тривиально и на $(X \times X) / G$, а это и означает, что пространство $X$ слабо симметрично относительно $\theta$.

\section{§ 4. Слабо коммутативные однородные пространства}

1. Векторное пространство $P$ с двумя билинейными операциями - умножением $(a, b) \mapsto a b$ и скобкой Пуассона $(a, b) \mapsto\{a, b\}$ называется алгеброй Пуассона, если вьполнены следуюшие условия:

1) умножение коммутативно и ассоциативно;

2) скобка Пуассона антикоммутативна и удовлетворяет тождеству Якоби;

3) умножение и скобка Пуассона связаны тождеством Лейбница

$$
\{a, b c\}=\{a, b\} c+b\{a, c\} .
$$

Обычно алгебры Пуассона возникают следуюшим образом. Пусть $A=\bigcup_{n=0}^{\infty} A^{(n)}$ - фильтрованная ассоциативная алгебра, т.е. $A^{(m)} A^{(n)} \subset A^{(m+n)}$. Рассмотрим ассоциированную с ней (ассоциативную) градуированную алгебру

$$
\operatorname{gr} A=P=\bigoplus_{n=0}^{\infty} P_{n}, \quad P_{n}=A^{(n)} / A^{(n-1)} \text {. }
$$

Предположим, что она коммутативна. Тогда в $P$ можно определить еше одну операцию, назьваемую скобкой Пуассона, по правилу

$$
\left\{a+A^{(m-1)}, b+A^{(n-1)}\right\}=a b-b a+A^{(m+n-2)} .
$$

При этом $\left\{P_{m}, P_{n}\right\} \subset P_{m+n-1}$ и алгебра $P$ с этими двумя операциями является алгеброй Пуассона.

Имея в виду эту конструкцию, можно сказать, что алгебра Пуассона - это "классический” объект, "квантовым" аналогом которого является некоммутативная ассоциативная алгебра.

В частности, алгебра Пуассона, ассоциированная с алгеброй $\mathscr{D}(X)$ дифференциальных операторов на многообразии $X$, - это алгебра $\mathscr{P}\left(T^{*} X\right)$ функций на кокасательном расслоении $T^{*} X$ многообразия $X$, полиномиальных на слоях (см. $\S 2$ ), с обычной операцией умножения и некоторой скобкой Пуассона. (См. об этом более подробно в п. 1.4 главы II.) 
Алгебра Пуассона называется коммутативной, если скобка Пуассона любых двух ее элементов равна нулю. Очевидно, что алгебра Пуассона, ассоциированная с фильтрованной коммутативной ассощиативной алгеброй, коммутативна. (Обратное, конечно, неверно.)

2. Пусть $G$ - вешественная группа Ли и $K$ - ее компактная подгруппа. Как и выше, мы будем предполагать, что многообразие $X$ связно.

ОПРЕДЕЛЕНИЕ 6. Однородное пространство $X=G / K$ назьвается слабо ком.мутативнылм, если алгебра Пуассона $\mathscr{P}\left(T^{*} X\right)^{G}$ коммутативна.

Напомним, что в силу предложения 7 алгебра $\mathscr{P}\left(T^{*} X\right)^{G}$ - это алгебра Пуассона, ассоциированная с алгеброй $\mathscr{D}(X)^{G}$ инвариантных дифференциальных операторов на $X$. Поэтому всякое коммутативное однородное пространство слабо коммутативно.

ТеОРема 5 [28], [22]. Всякое слабо коммутативное однородное пространство редуктивной группь Ли коммутативно.

Мы приведем доказательство этой теоремы в $\S 3$ следующей главы, а в $\S 4$ мы докажем аналогичную теорему для однородных пространств вида $(N \lambda K) / K$, где $N$ - (связная) нильпотентная группа Ли. По-видимому, вообще любое слабо коммутативное однородное пространство коммутативно. По крайней мере, автору не известно контрпримеров к этому утверждению.

3. Резюмируя эту главу, приведем схему включений рассмотренных классов однородных пространств.

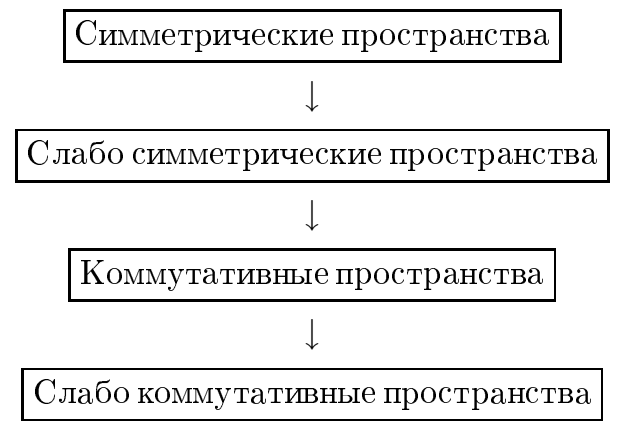

Для однородных пространств редуктивных групп последние три класса совпадают между собой (и совпадают с классом сферических пространств).

\section{Глава II. Коизотропные симплектические действия}

Имея в виду, что нас в основном интересуют однородные пространства групп Ли (и их кокасательные расслоения), мы будем предполагать все рассматриваемые многообразия аналитическими, хотя многие из приведенных в этой главе определений и фактов имеют смысл и верны для дифференцируемых многообразий.

Все рассматриваемые функции и отображения предполагаются аналитическими или являются таковьми.

Если не будет оговорено противное, мы будем рассматривать одновременно вещественные и комплексные многообразия. В случае необходимости основное поле будет обозначаться через $\mathbb{F}$. 
Допуская вольность речи, мы будем ради простоты выражений говорить о функциях на многообразии, имея в виду, как правило, функции, определенные на открытом подмножестве. Соответственно этому выражение "алгебра функций на многообразии $M$ ” будет означать “пучок алгебр функций на $M$ ”. То же относится и к тензорным полям.

\section{§1. Симплектические и пуассоновы многообразия}

Имеется много современных обзоров и монографий по симплектической геометрии и симплектическим действиям груп Ли. Из наиболее доступных русскому читателю упомянем обзоры [29] и [30] и книгу [31]. Нашей целью в этом и следуюшем параграфах будет лишш дать основные определения и сформулировать основные факты в удобной для нас форме и, в частности, фиксировать определения в случаях неоднозначности типа выбора знака.

1. Выпишем, прежде всего, некоторые основные формулы дифференциального исчисления на многообразиях.

Производная Ли тензорного поля $\tau$ по направлению векторного поля $\xi$ обозначается через $\xi \cdot \tau$. По определению

$$
\xi \cdot \tau=\left.\frac{d}{d t} g_{t} \tau\right|_{t=0}
$$

где $g_{t}$ - (локальная) однопараметрическая группа преобразований с полем скоростей $\xi$. В частности, если $\varphi-$ функция, то

$$
\xi \cdot \varphi=-\partial_{\xi} \varphi
$$

где $\partial_{\xi} \varphi$ - обычная производная функции $\varphi$ по направлению $\xi$; если $\eta$ - векторное поле, то

$$
\xi \cdot \eta=[\xi, \eta]
$$

где $[\xi, \eta]$ - коммутатор векторных полей $\xi$ и $\eta$. Для любых векторных полей $\xi, \eta$ и тензорного поля $\tau$ справедливо равенство

$$
\xi \cdot(\eta \cdot \tau)-\eta \cdot(\xi \cdot \tau)=[\xi, \eta] \cdot \tau
$$

Однопараметрическая група $g_{t}$ с полем скоростей $\xi$ сохраняет тензорное поле $\tau$ тогда и только тогда, когда $\xi \cdot \tau=0$. В этом случае мы будем говорить, что векторное поле $\xi$ аннулирует $\tau$.

Если $\alpha$ - дифференциальная 1-форма, то

$$
(d \alpha)(\xi, \eta)=\partial_{\xi} \alpha(\eta)-\partial_{\eta} \alpha(\xi)+\alpha([\xi, \eta])
$$

для любых векторных полей $\xi, \eta$; если $\omega$ - дифференциальная 2-форма, то

$$
\begin{aligned}
(d \omega)(\xi, \eta, \zeta)=\partial_{\xi} \omega(\eta, \zeta)+\partial_{\eta} \omega(\zeta, \xi) & +\partial_{\zeta} \omega(\xi, \eta) \\
& +\omega([\xi, \eta], \zeta)+\omega([\eta, \zeta], \xi)+\omega([\zeta, \xi], \eta)
\end{aligned}
$$

для любых векторных полей $\xi, \eta, \zeta$. 
2. Пусть $M$ - связное многообразие. Обозначим через $\mathscr{F}(M)$ алгебру функций на $M$ и через $\mathscr{V}(M)$ - алгебру Ли векторных полей на $M$.

Всякое поле бивекторов $B$ на $M$ определяет скобку $\{\cdot, \cdot\}$ в $\mathscr{F}(M)$ по формуле

$$
\{\varphi, \psi\}=B(d \varphi, d \psi)
$$

Это антикоммутативная билинейная операция, удовлетворяющая тождеству Лейбница. Легко видеть, что и, обратно, всякая операция с этими свойствами получается указанным способом.

Рассмотрим отображение

$$
v: \mathscr{F}(M) \rightarrow \mathscr{V}(M), \quad \varphi \mapsto B(\cdot, d \varphi) .
$$

Оно может быть иначе определено свойством

$$
v(\varphi) \cdot \psi=\{\varphi, \psi\}
$$

из чего ясно, что следующие условия эквивалентны:

(P1) скобка $\{\cdot, \cdot\}$ удовлетворяет тождеству Якоби;

(Р2) отображение $v$ является гомоморфизмом относительно скобки $\{\cdot, \cdot\}$ в $\mathscr{F}(M)$;

(P3) для любой функщии $\varphi$ векторное поле $v(\varphi)$ является дифференцированием скобки $\{\cdot, \cdot\}$ или, что эквивалентно, аннулирует поле бивекторов $B$.

Многообразие $M$ с полем бивекторов $B$, удовлетворяющим этим эквивалентным условиям, назьвается пуассоновым многообразием. Скобка $\{\cdot, \cdot\}$ в этом случае называется скобкой Пуассона. Алгебра $\mathscr{F}(M)$ со скобкой Пуассона в качестве второй операции является алгеброй Пуассона (см. определение в $\S 4$ главы I) и обозначается через $\mathscr{P}(M)$.

Векторные поля вида $v(\varphi)$ на пуассоновом многообразии $M$ назьваются гамильтоновыми. Они образуют алгебру Ли, определяющую некоторую (псевдо)групу Ли автоморфизмов пуассоновой структуры на $M$. Орбиты этой группы называются симплектическими слоями. Касательное пространство симплектического слоя в точке $p \in M$ образовано значениями всех гамильтоновых векторных полей и совпадает с носителем бивектора $B$ в этой точке.

Функции, постоянные на симплектических слоях, назьваются функииями Казими$p a$. Они образуют ядро гомоморфизма $v$ или, что то же, центр алгебры $\mathscr{P}(M)$ (относительно скобки Пуассона).

3. Если бивектор $B$ невырожден в каждой точке, то можно вместо него рассматривать 2-форму $\omega=\left(B^{\top}\right)^{-1}$. Из $(24)$ и $(25)$ следует, что тогда

$$
\{\varphi, \psi\}=\omega(v(\varphi), v(\psi)) .
$$

Подставляя в формулу $(23) \xi=v(\varphi), \eta=v(\psi), \zeta=v(\chi)$, получаем:

$$
-d \omega(\xi, \eta, \zeta)=\{\{\varphi, \psi\}, \chi\}+\{\{\psi, \chi\}, \varphi\}+\{\{\chi, \varphi\}, \psi\},
$$

так что тождество Якоби для скобки $\{\cdot, \cdot\}$ равносильно замкнутости формы $\omega$.

Связное многообразие $M$ с заданной на нем невырожденной замкнутой 2-формой $\omega$ называется симплектическим многообразием. В силу предыдушего симплектические многообразия - это частный случай пуассоновых многообразий. 
Если $M$ - симплектическое многообразие, то векторное поле $v(\varphi)(\varphi \in \mathscr{F}(M))$ может быть определено равенством

$$
\omega(v(\varphi), \eta)=\partial_{\eta} \varphi \quad(\eta \in \mathscr{V}(M))
$$

и по этой причине называется косым градиентом функции $\varphi$. Оно обычно обозначается через $\operatorname{sgrad} \varphi$.

Каждому векторному полю $\xi$ на симплектическом многообразии $M$ поставим в соответствие 1-форму $\alpha=\omega(\xi, \cdot)$. С помошью формул (22) и (23) нетрудно доказать, что $\xi \cdot \omega=-d \alpha$. В частности, $\xi \cdot \omega=0$ тогда и только тогда, когда форма $\alpha$ замкнута. В этом случае $\alpha$ локально представляется в виде $\alpha=d \varphi, \varphi \in \mathscr{F}(M)$, причем из $(28)$ следует, что $\xi=v(\varphi)$. Таким образом, всякое векторное поле, аннулирующее форму $\omega$, локально гамильтоново.

Так как на симплектическом многообразии значения гамильтоновых векторных полей в любой точке составляют все касательное пространство, то ядро гомоморфизма $v$ состоит только из констант. Это означает, что функция $\varphi$, для которой $v(\varphi)=\xi$, определяется векторньм полем $\xi$ однозначно с точностью до аддитивной константы. Всякая такая функция $\varphi$ назьвается гамильтонианом поля $\xi$.

Простейшим примером симплектического многообразия является симплектическое векторное пространство (с постоянной 2-формой). По теореме Дарбу всякое симплектическое многообразие локально изоморфно симплектическому векторному пространству.

Симплектические слои произвольного пуассонова многообразия $M$ имеют естественную симплектическую структуру. При этом скобка Пуассона любых двух функций на $M$ может вычисляться "послойно", т.е. ее ограничение на любой симплектический слой совпадает со скобкой Пуассона ограничений этих функщий на данньй слой.

4. В $\S 4$ главы I была определена пуассонова структура на кокасательном расслоении $T^{*} X$ произвольного многообразия $X$. Опишем ее более явно.

Сделаем, прежде всего, замечание, касаюшееся выбора знака. А именно, будем считать, что изоморфизм между алгебрами $\operatorname{gr} \mathscr{D}(X)$ и $\mathscr{P}\left(T^{*} X\right)$ установлен таким образом, что векторное поле $\xi$ на $X$, рассматриваемое как линейная функция на $T^{*} X$, отвечает в алгебре gr $\mathscr{D}(X)$ дифференцированию Ли по направлению $\xi$, т.е. обычному дифференщированию по направлению $\xi$, взятому со знаком минус.

Ввиду тождества Лейбница достаточно знать скобку Пуассона функций, постоянных на слоях канонического отображения $p: T^{*} X \rightarrow X$, и функций, линейных на слоях. Функции, постоянные на слоях, - это функции вида $p^{*} \varphi$, где $\varphi \in \mathscr{F}(X)$. Им соответствуют операторы умножения на функцию. Функции, линейные на слоях, - это векторные поля на $X$. Им соответствуют дифференциальные операторы первого порядка, описанные вьше. Рассматривая коммутаторы этих дифференциальных операторов, получаем следуюшие формулы:

$$
\begin{gathered}
\left\{p^{*} \varphi, p^{*} \psi\right\}=0 \quad(\varphi, \psi \in \mathscr{F}(X)), \\
\left\{p^{*} \varphi, \eta\right\}=p^{*}\left(\partial_{\eta} \varphi\right) \quad(\varphi \in \mathscr{F}(X), \eta \in \mathscr{V}(X)), \\
\{\xi, \eta\}=[\xi, \eta] \quad(\xi, \eta \in \mathscr{V}(X)) .
\end{gathered}
$$

Найдем теперь гамильтоновы векторные поля на $T^{*} X$, отвечаюшие функциям рассмотренного вида. 
Можно выделить два класса векторных полей на $T^{*} X$. Во-первых, это вертикальные векторные поля, касающиеся слоев отображения $p$. Во-вторых, всякое преобразование многообразия $X$ каноническим образом поднимается до преобразования многообразия $T^{*} X$ и в соответствии с этим каждое векторное поле $\xi \in \mathscr{V}(X)$ каноническим образом поднимается до векторного поля $\widehat{\xi} \in \mathscr{V}\left(T^{*} X\right)$; при этом $\widehat{[\xi, \eta]}=[\widehat{\xi}, \widehat{\eta}]$. Получаемые таким образом векторные поля на $T^{*} X$ будем называть структурными.

Согласно определению (26) из формул (29)-(31) вытекает, что

$$
\begin{aligned}
v\left(p^{*} \varphi\right) & =-d \varphi, \\
v(\xi) & =\widehat{\xi} .
\end{aligned}
$$

(Здесь $d \varphi$ - вертикальное векторное поле, равное $d_{x} \varphi$ во всех точках слоя $T_{x}(X)$.)

Мы видим, в частности, что гамильтоновы векторные поля порождают касательное пространство в каждой точке многообразия $T^{*} X$. Следовательно, рассматриваемая пуассонова структура на самом деле является симплектической. Пользуясь формулой (27), из формул (29)-(33) получаем следующее описание соответствующей 2-формы $\omega$ :

$$
\begin{gathered}
\omega(\gamma, \delta)=0, \\
\omega(\widehat{\xi}, \delta)=\delta(\xi), \\
\omega(\widehat{\xi}, \widehat{\eta})=[\xi, \eta]
\end{gathered}
$$

для любых вертикальных векторных полей $\gamma, \delta$ и структурных векторных полей $\widehat{\xi}, \widehat{\eta}$.

5. Другая важная конструкция пуассоновых многообразий связана с рассмотрением естественной фильтрации

$$
U(\mathfrak{g})=\bigcup_{n=0}^{\infty} U^{(n)}(\mathfrak{g})
$$

в обертывающей алгебре $U(\mathfrak{g})$ алгебры Ли $\mathfrak{g}$ (см. п. 2.5 главы I).

Градуированная алгебра, ассоциированная с этой фильтрацией алгебры $U(\mathfrak{g})$, есть симметрическая алгебра $S(\mathfrak{g})$ с естественной градуировкой

$$
S(\mathfrak{g})=\bigoplus_{n=0}^{\infty} S^{n}(\mathfrak{g})
$$

Так как она коммутативна, то операция коммутирования в $U(\mathfrak{g})$ порождает в ней скобку Пуассона, преврашающую ее в градуированную алгебру Пуассона

$$
P(\mathfrak{g})=\bigoplus_{n=0}^{\infty} P_{n}(\mathfrak{g}) .
$$

Мы будем называть эту алгебру Пуассона алгеброй Пуассона-Ли-Березина. 
Так как $P(\mathfrak{g})$ порождается (в смысле операции умножения) подпространством $P_{1}(\mathfrak{g})=S^{1}(\mathfrak{g})=\mathfrak{g}$, то для описания скобки Пуассона в ней достаточно указать скобку Пуассона любых элементов $\xi, \eta \in \mathfrak{g}$. Из определения очевидно, что

$$
\{\xi, \eta\}=[\xi, \eta] .
$$

Отождествим алгебру $S(\mathfrak{g})$ с алгеброй $\mathbb{F}\left[\mathfrak{g}^{*}\right]$ полиномиальных функций на сопряженном пространстве $\mathfrak{g}^{*}$ алгебры $\mathfrak{g}$. Тогда определенная вьше скобка Пуассона превращает $\mathfrak{g}^{*}$ в пуассоново многообразие. Из (37) следует, что

$$
\{\varphi, \psi\}=[d \varphi, d \psi]
$$

для любых функций $\varphi, \psi$ на $\mathfrak{g}^{*}$.

Из формул (26) и (37) следует, что гамильтоново векторное поле $v(\xi)$ на $\mathfrak{g}^{*}$, отвечающее элементу $\xi \in \mathfrak{g}$ как линейной функции на $\mathfrak{g}^{*}$, имеет вид

$$
v(\xi)(\alpha)=\operatorname{ad}^{*}(\xi) \alpha \quad\left(\alpha \in \mathfrak{g}^{*}\right),
$$

где $\mathrm{ad}^{*}$ обозначает коприсоединенное представление алгебры Ли g. Иначе говоря, $v(\xi)$ - это поле скоростей коприсоединенного представления. Любое гамильтоново векторное поле является линейной комбинацией полей $v(\xi), \xi \in \mathfrak{g}$, с функциональными коэф фициентами. Отсюда следует, что симплектические слои пуассонова многообразия $\mathfrak{g}^{*}$ - это орбиты коприсоединенной группы.

В силу $(27),(37)$ и (39) симплектическая структура на коприсоединенной орбите задается формой $\omega$, определяемой в точке $\alpha \in \mathfrak{g}^{*}$ по формуле

$$
\omega\left(\operatorname{ad}^{*}(\xi) \alpha, \operatorname{ad}^{*}(\eta) \alpha\right)=\alpha([\xi, \eta]) .
$$

Эта форма назьвается формой Костанта-Кириллова.

6. Пуассонову структуру (в отличие от симплектической) можно факторизовать по действию групшы.

Пусть $M$ - пуассоново многообразие, и пусть задано действие групшы Ли $H$ на $M$, сохраняющее пуассонову структуру. Предположим, что существуют многообразие $N=M / H$ и отображение $\pi=\pi_{H}: M \rightarrow N$ (которое мы будем называть факторизацией) со следуюшими свойствами:

1) слои $\pi$ - это в точности орбиты групшы $H$;

2) подмножество $U \subset N$ открыто тогда и только тогда, когда подмножество $\pi^{-1}(U) \subset M$ открыто;

$3)$ функция $\varphi$ на $N$ аналитична тогда и только тогда, когда функщия $\pi^{*} \varphi$ на $M$ аналитична.

Тогда $\pi^{*}$ осуществляет изоморфизм алгебры $\mathscr{F}(N)$ аналитических функций на $N$ и алгебры $\mathscr{F}(M)^{H} H$-инвариантных аналитических функций на $M$. (Напомним, что согласно нашей договоренности выражение "алгебра функций" - это эвфемизм, заменяющий выражение "пучок алгебр функций".) Поскольку алгебра $\mathscr{F}(M)^{H}=\mathscr{P}(M)^{H}$ есть подалгебра алгебры $\mathscr{P}(M)$, это наделяет алгебру $\mathscr{F}(N)$ структурой алгебры Пуассона и, тем самым, определяет пуассонову структуру на многообразии $N$. Соответствуюшее поле бивекторов есть просто (корректно определенньй) образ поля бивекторов, определяющего пуассонову структуру на $M$. 
Невырожденность пуассоновой структуры, вообще говоря, не сохраняется при факторизации. Это, в частности, будет хорошо видно на приводимом ниже примере.

Пусть $G$ - связная группа Ли. Будем считать, что она действует на себе правыми сдвигами. Это действие индуцирует действие $G: T^{*} G$, сохраняющее каноническую симплектическую структуру кокасательного расслоения. Отображение

$$
\pi: T^{*} G \rightarrow \mathfrak{g}^{*}
$$

ставящее в соответствие каждой линейной форме $\alpha \in T_{g}^{*}(G)$ ее сдвиг в единищу группы $G$, является факторизацией в определенном вьше смысле. Тем самым, на $\mathfrak{g}^{*}$ индуцируется пуассонова структура.

Докажем, что эта структура совпадает с пуассоновой структурой на $\mathfrak{g}^{*}$, определенной в п. 5. Для этого достаточно проверить совпадение скобок Пуассона линейных функций, т.е. элементов алгебры $\mathfrak{g}$.

Для любой функции $\varphi$ на $\mathfrak{g}^{*}$ обозначим через $\varphi^{(r)}$ правоинвариантную функцию на $T^{*} G$, ограничение которой на $T_{e}^{*}(G)=\mathfrak{g}^{*}$ совпадает с $\varphi$. При $\xi, \eta \in \mathfrak{g}$ в силу (31) получаем:

$$
\{\xi, \eta\}=\left\{\xi^{(r)}, \eta^{(r)}\right\}(e)=\left[\xi^{(r)}, \eta^{(r)}\right](e)=[\xi, \eta],
$$

что согласуется с (37).

Теперь мы в состоянии вычислить скобку Пуассона любых правоинвариантных функций на $T^{*} G$. А именно, согласно (38) имеем

$$
\left\{\varphi^{(r)}, \psi^{(r)}\right\}=[d \varphi, d \psi]^{(r)} .
$$

Более подробно,

$$
\left\{\varphi^{(r)}, \psi^{(r)}\right\}(\alpha)=\alpha\left(\left[d_{\alpha} \varphi, d_{\alpha} \psi\right]\right)
$$

для любого $\alpha \in \mathfrak{g}^{*}$.

Такая же формула, но со знаком минус имеет место для скобки Пуассона левоинвариантных функций на $T^{*} G$.

7. Подмногообразие симплектического многообразия не является, вообще говоря, симплектическим многообразием, так как ограничение структурной 2-формы на нем может быть вырожденньм. Тем не менее, для некоторых функций на подмногообразии все же можно определить скобку Пуассона.

Пусть, вообще, $N$ - многообразие с заданной на нем замкнутой (но не обязательно невырожденной) 2-формой $\omega$. Предположим, что форма $\omega$ имеет постоянньй ранг. Из формулы (23) следует, что векторные поля, значения которых в каждой точке принадлежат ядру формы $\omega$, аннулируют $\omega$ и образуют алгебру Ли. Они порождают некоторую (псевдо)группу Ли преобразований $H$ многообразия $N$. Орбиты этой групшы будем назьвать слоями вырождения.

Пусть $\mathscr{F}(N)^{0}$ - алгебра функщий на $N$, постоянных на слоях вырождения. Для любых функций $\varphi, \psi \in \mathscr{F}(N)^{0}$ можно корректно определить их скобку Пуассона, руководствуясь формулами $(27)$ и (28). Тем самым, алгебра $\mathscr{F}(N)^{0}$ преврашается в алгебру Пуассона, которую мы обозначим через $\mathscr{P}(N)^{0}$.

Предположим теперь, что существует факторизация

$$
\pi_{H}: N \rightarrow N^{0}=N / H
$$


в смысле п. 6. Тогда алгебра $\mathscr{F}\left(N^{0}\right)$ канонически отождествляется с алгеброй $\mathscr{F}(N)^{0}$ и, тем самым, многообразие $N^{0}$ наделяется пуассоновой структурой. Легко понять, что эта структура невырожденна. Симплектическое многообразие $N^{0}$ будем называть редукиией многообразия $N$.

Применим вьшеизложенное к некоторьм “большим" подмногообразиям симплектических многообразий.

Подпространство симплектического векторного пространства называется изотропным, если ограничение структурной 2-формы на нем тождественно равно нулю, и коизотропнылм, если его ортогональное дополнение (в смысле структурной 2-формы) изотропно. Изотропное подпространство может быть также определено как подпространство, содержашееся в своем ортогональном дополнении, а коизотропное - как подпространство, содержащее свое ортогональное дополнение.

Подмногообразие $N$ симплектического многообразия $M$ назьвается коизотроn$\boldsymbol{H} \boldsymbol{\text { м }}$, если подпространство $T_{p}(N)$ коизотропно в $T_{p}(M)$ для каждой точки $p \in N$.

Ограничение структурной 2-формы многообразия $M$ на коизотропное подмногообразие $N$ имеет постоянный ранг (равный $2 \operatorname{dim} N-\operatorname{dim} M)$. Если $\varphi, \psi \in \mathscr{P}(M)-$ такие функции, что их ограничения на $N$ принадлежат $\mathscr{P}(N)^{0}$, то векторные поля $v(\varphi), v(\psi)$ ортогональны слоям вырождения подмногообразия $N$ и в силу коизотропности последнего касаются его. Отсюда следует, что

$$
\left.\{\varphi, \psi\}\right|_{N}=\left\{\left.\varphi\right|_{N},\left.\psi\right|_{N}\right\} .
$$

8. Рассмотрим важньй частный случай описанной вьше ситуации.

Пусть $H$ - подгруппа Ли групшы Ли $G$. Будем считать, что $H$ действует на $G$ правыми сдвигами, и рассмотрим подрасслоение $T_{H}^{*} G \subset T^{*} G$, образованное аннуляторами касательных пространств $H$-орбит. Его касательное пространство в любой точке порождается касательным пространством слоя и скоростями действия $G: T^{*} G$ левыми сдвигами. Оно также содержит скорости действия $H: T^{*} G$ правыми сдвигами, причем, как следует из формул (35) и (36), последние ортогональны ему. По соображениям размерности мы можем заключить, что $T_{H}^{*} G$ - коизотропное подмногообразие в $T^{*} G$, причем его слоями вырождения являются $H$-орбиты. Легко понять, что редукция многообразия $T_{H}^{*} G-$ это $T^{*}(G / H)$.

Это позволяет получить удобную формулу для скобки Пуассона любых $G$-инвариантных функций на $T^{*}(G / H)$, обобщаюшую формулу (42).

Условимся отождествлять каноническим образом касательное пространство $T_{o}(G / H)$ многообразия $G / H$ в точке $o=e H$ с пространством $\mathfrak{g} / \mathfrak{h}$, а кокасательное пространство $T_{o}^{*}(G / H)$ - с пространством $(\mathfrak{g} / \mathfrak{h})^{*}$, канонически вложенньп в $\mathfrak{g}^{*}$ (в виде аннулятора $\mathfrak{h}^{\circ}$ подалгебры $\left.\mathfrak{h}\right)$.

Для любой $H$-инвариантной функции $\varphi$ на $(\mathfrak{g} / \mathfrak{h}) *$ обозначим через $\widetilde{\varphi} G$-инвариантную функцию на $T^{*}(G / H)$, ограничение которой на $T_{o}^{*}(G / H)=(\mathfrak{g} / \mathfrak{h})^{*}$ совпадает с $\varphi$. Прообраз функции $\widetilde{\varphi}$ на $T_{H}^{*} G$ является $G$-левоинвариантной функцией, постоянной на слоях вырождения. В силу этого из формулы (43) и левоинвариантного варианта формулы (42) следует, что

$$
\{\widetilde{\varphi}, \widetilde{\psi}\}(\alpha)=-\alpha\left(\left[d_{\alpha} \varphi, d_{\alpha} \psi\right]\right) \quad\left(\alpha \in(\mathfrak{g} / \mathfrak{h})^{*}\right)
$$

для любых $H$-инвариантных функций $\varphi, \psi$ на $(\mathfrak{g} / \mathfrak{h})^{*}$. Коммутатор в правой части этой формулы следует понимать как коммутатор в $\mathfrak{g}$ любых представителей смежных классов $d_{\alpha} \varphi, d_{\alpha} \psi \in \mathfrak{g} / \mathfrak{h}$ (от выбора которых результат не зависит). 


\section{§ 2. Пуассоновы действия и отображение моментов}

1. Действие связной группы Ли $G$ на пуассоновом многообразии $M$ назьвается $n y$ ассоновым, если задан гомоморфизм алгебр Ли (называемый гамильтонианом действия)

$$
\mathfrak{g} \rightarrow \mathscr{P}(M), \quad \xi \mapsto H_{\xi},
$$

удовлетворяюший условию

$$
v\left(H_{\xi}\right)=\xi_{*},
$$

где $\xi_{*}$ - поле скоростей действия, отвечающее элементу $\xi \in \mathfrak{g}$. При этом автоматически данное действие сохраняет пуассонову структуру и

$$
H_{\mathrm{Ad}(g) \xi}=g H_{\xi}
$$

для любого $g \in G$. В случае несвязной группы $G$ вьполнение последних двух свойств естественно потребовать дополнительно.

ПримеР 1. Пусть группа Ли $G$ действует на многообразии $X$. Тогда в силу $(31)$ и (33) индуцированное действие $G: T^{*} X$ является пуассоновым с гамильтонианом

$$
H_{\xi}=\xi_{*},
$$

где $\xi_{*}$ - поле скоростей действия $G: X$, рассматриваемое как функция на $T^{*} X$, линейная на слоях.

ПримеР 2. Симплектическое линейное представление групшы Ли $G$ в векторном пространстве $V$ является пуассоновым действием с квадратичным гамильтонианом

$$
H_{\xi}(x)=\frac{1}{2} \omega(\xi x, x) \quad(x \in V) .
$$

ПримеР 3. Пусть задано произвольное линейное представление группы Ли $G$ в векторном пространстве $U$. Определим в пространстве $V=U \oplus U^{*}$ невырожденную кососимметрическую билинейную форму $\omega$ следуюшим каноническим образом:

$$
\begin{gathered}
\omega(U, U)=\omega\left(U^{*}, U^{*}\right)=0, \\
\omega(x, \alpha)=\alpha(x) \quad\left(x \in U, \alpha \in U^{*}\right) .
\end{gathered}
$$

Естественное действие $G: V$ сохраняет эту форму и согласно примеру 2 является пуассоновым. С другой стороны, пространство $V$ можно рассматривать как $T^{*} U$, и поэтому то же действие является пуассоновым согласно примеру 1 . Нетрудно видеть, что гамильтонианы (47) и (48) в данном случае совпадают.

ПРИМЕР 4. В силу формул (37) и (39) коприсоединенное представление групшы Ли $G$ является пуассоновым действием с гамильтонианом

$$
H_{\xi}=\xi
$$

относительно пуассоновой структуры в $\mathfrak{g}^{*}$, описанной в п. 1.5.

2. Действие группы Ли $G$ на симплектическом многообразии $M$ называется $c u м$ плектическим, если оно сохраняет структурную форму $\omega$. 
ПРЕДЛОЖЕНИЕ 1. Если группа Ли $G$ полупроста или, более общо, если $H^{1}(\mathfrak{g}, \mathbb{F})=H^{2}(\mathfrak{g}, \mathbb{F})=0, \quad$ то всякое симплектическое действие группь $G$ является пуассоновым.

ДокАЗАТЕльство. Для всякой точки $p \in M$ рассмотрим $G$-эквивариантное отображение

$$
G \rightarrow M, \quad g \mapsto g p .
$$

Прообраз формы $\omega$ при этом отображении является замкнутой левоинвариантной 2-формой на групе $G$, которая в силу условия $H^{2}(\mathfrak{g}, \mathbb{F})=0$ должна быть дифференциалом левоинвариантной 1-формы. Это означает, что существует такая (однозначно определенная) 1-форма $\alpha_{p}$ на $\mathfrak{g}$, что

$$
\omega\left(\xi_{*}(p), \eta_{*}(p)\right)=\alpha_{p}([\xi, \eta])
$$

для любых $\xi, \eta \in \mathfrak{g}$. Положим $H_{\xi}(p)=\alpha_{p}(\xi)$; тогда

$$
H_{[\xi, \eta]}=\omega\left(\xi_{*}, \eta_{*}\right)
$$

Для любых $\xi, \eta, \zeta \in \mathfrak{g}$ имеем в силу $(28)$ и $(23)$ :

$$
\omega\left(v\left(H_{[\xi, \eta]}\right), \zeta_{*}\right)=-\zeta_{*} \cdot \omega\left(\xi_{*}, \eta_{*}\right)=-\omega\left([\zeta, \xi]_{*}, \eta_{*}\right)-\omega\left(\xi_{*},[\zeta, \eta]_{*}\right)=\omega\left([\xi, \eta]_{*}, \zeta_{*}\right),
$$

откуда $v\left(H_{[\xi, \eta]}\right)=[\xi, \eta]_{*}$. Так как $\mathfrak{g}=[\mathfrak{g}, \mathfrak{g}]$, то $v\left(H_{\xi}\right)=\xi_{*}$ для любого $\xi \in \mathfrak{g}$. Наконец, так как отображение $\eta \mapsto H_{\eta} G$-эквивариантно, то

$$
\left\{H_{\xi}, H_{\eta}\right\}=\xi_{*} \cdot H_{\eta}=H_{[\xi, \eta]} \text {. }
$$

3. Для всякого пуассонова действия $G: M$ определено так называемое отображение моментов

$$
\Phi: M \rightarrow \mathfrak{g}^{*}, \quad \Phi(p)(\xi)=H_{\xi}(p) \quad(p \in M, \xi \in \mathfrak{g}) .
$$

Более наглядно его можно представлять себе следуюшим образом. Пусть $\left\{\xi_{1}, \ldots, \xi_{n}\right\}$ - базис алгебры Ли $\mathfrak{g}$ и $H_{1}, \ldots, H_{n}$ - гамильтонианы базисных векторов. Тогда $\Phi(p)$ есть точка пространства $\mathfrak{g}^{*}$ с координатами $H_{1}(p), \ldots, H_{n}(p)$.

Отображение $\varphi: M \rightarrow N$ пуассоновых многообразий будем называть морфизмом, если соответствуюший обратньй гомоморфизм $\varphi^{*}$ алгебр функций сохраняет скобку Пуассона, т.е. является гомоморфизмом алгебр Пуассона.

ПРЕДЛОЖЕНИЕ 2. Отображение моментов $\Phi: M \rightarrow \mathfrak{g}^{*}$ является әквивариантным морфизмом пуассоновых многообразий.

ДоказАТЕльство. Эквивариантность отображения $\Phi$ очевидна. Для доказательства того, что оно является морфизмом пуассоновых многообразий, достаточно проверить, что $\Phi^{*}$ сохраняет скобку Пуассона линейных функций на $\mathfrak{g}^{*}$. Из определения (49) следует, что $\Phi^{*}(\xi)=H_{\xi}$ при $\xi \in \mathfrak{g}$. Для любых $\xi, \eta \in \mathfrak{g}$ имеем в силу (37):

$$
\Phi^{*}(\{\xi, \eta\})=\Phi^{*}([\xi, \eta])=H_{[\xi, \eta]}=\left\{H_{\xi}, H_{\eta}\right\}=\left\{\Phi^{*}(\xi), \Phi^{*}(\eta)\right\} .
$$


ПримеР 5. Для пуассонова действия $G: T^{*} X$ из примера 1 отображение моментов вьглядит следующим образом:

$$
\Phi(\alpha)(\xi)=\alpha\left(\xi_{*}(x)\right) \quad\left(\alpha \in T_{x}^{*}(X)\right) .
$$

В частности, пусть $X=G / H$. Положим $o=e H$ и условимся каноническим образом отождествлять пространство $T_{o}^{*}(X)$ с пространством $(\mathfrak{g} / \mathfrak{h})^{*}$, вложенньм в $\mathfrak{g}^{*}$ в виде аннулятора $\mathfrak{h}^{\circ}$ подалгебры $\mathfrak{h}$. Тогда

$$
\Phi(\alpha)=\alpha \quad\left(\alpha \in(\mathfrak{g} / \mathfrak{h})^{*} \subset \mathfrak{g}^{*}\right)
$$

ПримеР 6. Рассмотрим пуассоново действие $G: V$ из примера 2. Его дифференциал есть гомоморфизм $\rho: \mathfrak{g} \rightarrow \mathfrak{s p}(V)$. Отождествим алгебру Ли $\mathfrak{s p}(V)$ с ее сопряженным пространством при помоши скалярного умножения $(A, B)=\operatorname{tr} A B$ и рассмотрим линейное отображение

$$
\rho^{*}: \mathfrak{s p}(V) \rightarrow \mathfrak{g}^{*},
$$

сопряженное отображению $\rho$. Далее, любым двум векторам $u, v \in V$ поставим в соответствие линейньй оператор

$$
x \mapsto \frac{1}{2}(\omega(x, u) v+\omega(x, v) u) .
$$

Тем самьм будет определен изоморфизм

$$
\sigma: S^{2} V \stackrel{\sim}{\sim} \mathfrak{s p}(V) .
$$

Нетрудно проверить, что

$$
\Phi(v)=\frac{1}{2} \rho^{*}\left(\sigma\left(v^{2}\right)\right) .
$$

ПримеР 7. В условиях предложения $1 \Phi(p)=\alpha_{p}$, где $\alpha_{p}$ - линейная форма на $\mathfrak{g}$, определенная в доказательстве этого предложения.

4. Вычислим дифференциал отображения моментов для пуассонова действия группы Ли $G$ на симплектическом многообразии $M$. В силу (28) имеем:

$$
d \Phi(p, d p)(\xi)=\omega\left(\xi_{*}(p), d p\right) .
$$

Отсюда следует, что

$$
\operatorname{Ker} d_{p} \Phi=\mathfrak{g}_{*}(p)^{\perp},
$$

где знак $\perp$ обозначает ортогональное дополнение в смысле формы $\omega$.

В частности, если действие $G: M$ транзитивно, то $\mathfrak{g}_{*}(p)=T_{p}(M)$ и $\operatorname{Ker} d_{p} \Phi=0$ для любой точки $p \in M$. Отсюда получаем 
ПРЕДЛОЖЕНИЕ 3. При сделанных выше предположениях, если действие $G$ : $M$ транзитивно, то отображение моментов является әквивариантным симплектическим накрытием над некоторой орбитой коприсоединенного представления группьи $G$.

В обшем случае из формулы (56) следует, что размерность образа отображения моментов равна размерности орбиты общего положения действия $G: M$.

5. Пусть задано пуассоново действие группы Ли $G$ на симплектическом многообразии $M$. Рассмотрим алгебру функций

$$
\mathscr{A}(M)=\Phi^{*}\left(\mathscr{P}\left(\mathfrak{g}^{*}\right)^{G}\right) \subset \mathscr{P}(M) .
$$

Так как $\mathscr{P}\left(\mathfrak{g}^{*}\right)^{G}$ - это центр алгебры Пуассона $\mathscr{P}\left(\mathfrak{g}^{*}\right)$ (см. п. 1.2), то алгебра $\mathscr{A}(M)$ коммутативна относительно скобки Пуассона. Соответствующие гамильтоновы векторные поля обладают следуюшими свойствами:

1) они коммутируют друг с другом;

2) они $G$-инвариантны;

3) они ортогональны орбитам, так как происходят из функций, постоянных на орбитах;

4) они касательны орбитам, так как происходят из функций, постоянных на слоях отображения моментов.

Более точно, значения этих векторных полей в точке $p \in M$ общего положения образуют подпространство

$$
\left.\operatorname{Ker} d \Phi\right|_{\mathfrak{g}_{*}(p)}=\left.\operatorname{Ker} \omega\right|_{\mathfrak{g}_{*}(p)} \subset T_{p}(M) .
$$

Указанные векторные поля порождают некоторую абелеву (псевдо)групу $G$-эквивариантных автоморфизмов многообразия $M$, сохраняющих орбиты и назьваемых инвариантными коллективными движениями. Ее ограничение на орбиту $G p=$ $G / G_{p}$ есть некоторая абелева подгруппа группы $N\left(G_{p}\right) / G_{p}$, действующей на $G / G_{p}$ правыми сдвигами. Из (58) следует, что для точки $p$ общего положения эта подгруппа совпадает со связной компонентой групшы $G_{\Phi(p)} / G_{p}$ и, значит,

$$
\mathfrak{g}_{\Phi(p)} \supset \mathfrak{g}_{p} \supset\left[\mathfrak{g}_{\Phi(p)}, \mathfrak{g}_{\Phi(p)}\right] .
$$

ОПРЕДЕЛЕНИЕ 1. Назовем дефектом симплектического действия $G: M$ дефект ограничения формы $\omega$ на касательное пространство орбиты обшего положения.

Дефект действия $G: M$ будем обозначать через $\operatorname{def}(M, G)$ или $\operatorname{def} M$.

Из (58) следует, что дефект пуассонова действия равен $\operatorname{dim} G_{\Phi(p)} / G_{p}$ для точки $p$ обшего положения. С другой стороны, так как $\operatorname{dim} \Phi(M)=\operatorname{dim} G / G_{p}$, то дефект действия $G: M$ равен коразмерности орбиты общего положения для действия $G: \Phi(M)$.

6. Рассмотрим случай редуктивной группы Ли $G$ (см. определение в п. 3.1 главы I). В этом случае имеется возможность отождествить пространства $\mathfrak{g}$ и $\mathfrak{g}^{*}$ при помощи какого-нибудь инвариантного скалярного умножения. Имея это в виду, будем считать, что отображение моментов $\Phi$ есть отображение из $M$ в $\mathfrak{g}$. Подгруппа $G_{\Phi(p)}$ будет тогда централизатором элемента $\Phi(p) \in \mathfrak{g}$ в $G$.

Пусть $\pi_{G}: \mathfrak{g} \rightarrow \mathfrak{g} / / G$-морфизм факторизации. Известно, что каждьй его слой есть объединение конечного числа орбит групшы $G$.

Отсюда следует 
ПреДЛОЖЕнИЕ 4. Дефект пуассонова действия редуктивной группы Ли G на симплектическом многообразии $M$ равен $\operatorname{dim} \pi_{G}(\Phi(M))$.

ЛЕмма 1. Для любого неприводимого по Зарисскому подмножсества $S \subset \mathfrak{g}$ имеет место ровно одна из следующих двух возможностей:

1) некоторое непустое открытое по Зарисскому подмножество в $S$ состоит из полупростых әлементов;

2) все полупростые әлементы в $S$ содержатся в собственном замкнутом по Зарисскому подмножестве.

ДокАЗАТЕЛЬСтво. Алгебра g покрывается конечным числом $n л а с т о в-$ неприводимых компонент алгебраических подмногообразий, образованных всеми $G$-орбитами (классами сопряженности) данной размерности. Существует пласт, содержаший непустое открытое по Зарисскому подмножество множества $S$. Однако известно, что если в пласте присутствуют полупростые элементы, то они составляют в нем открытое по Зарисскому подмножество [32]. Отсюда и следует утверждение леммы.

ОПРЕДЕЛЕНИЕ 2. Пуассоново действие редуктивной группы Ли $G$ на симплектическом многообразии $M$ назовем симплектически стабильныл, если некоторое непустое открытое по Зарисскому подмножество в $\Phi(M)$ состоит из полупростых элементов.

Централизатор полупростого элемента в алгебре $\mathfrak{g}$ является редуктивной подалгеброй максимального ранга, система корней которой есть пересечение системы корней алгебры $\mathfrak{g}$ с некоторым подпространством. Такие подалгебры иногда называют подалгебрами Леви.

В случае симплектически стабильного действия $G: M$ для точки $p \in M$ общего положения подалгебра $\mathfrak{g}_{\Phi(p)}$ является подалгеброй Леви, а подалгебра $\mathfrak{g}_{p}$ есть сумма ее коммутанта и некоторой подалгебры ее центра. Поэтому

$$
\operatorname{def} M=\operatorname{rk} \mathfrak{g}-\mathrm{rk} \mathfrak{g}_{p} .
$$

В частности, дефект симплектически стабильного действия определяется стабилизатором точки общего положения.

Для действий на кокасательных расслоениях симплектическая стабильность может быть установлена при помоши следуюшего результата $\Phi$. Кнопа.

Алгебраическое многообразие назьвается квазиаффиннылм, если оно изоморфно открытому подмногообразию афффинного многообразия.

Tеорема 1 [33]. Пусть редуктивная алгебраическая группа $G$ действует на неприводимом гладком квазиаффинном алгебраическом многообразии X. Тогда действие $G: T^{*} X$ симплектически стабильно.

Эта теорема была доказана в [33] для комплексных алгебраических групш и многообразий, но она верна и в вешественном случае, поскольку из леммы 1 следует, что симплектическая стабильность (или ее отсутствие) сохраняется при комплексификации. Ее доказательство будет приведено в п. 3.9. 


\section{§3. Коранг симплектического действия}

1. Пусть $(V, \omega)$ - симплектическое векторное пространство.

Подпространство $U \subset V$ назовем невырожденныл, если ограничение формы $\omega$ на нем невырожденно. Это равносильно тому, что $V=U \oplus U^{\perp}$, где знак $\perp$ обозначает ортогональное дополнение в смысле формы $\omega$.

В общем случае ранг ограничения формы $\omega$ на $U$ назовем рангом подпространства $U$ и обозначим через $\operatorname{rk} U$. В частности, подпространства ранга 0 - это изотропные подпространства (см. п. 1.7). Число $\mathrm{rk} U^{\perp}$ назовем корангом подпространства $U$ и обозначим через $\operatorname{cork} U$. В частности, подпространства коранга 0 - это коизотропные подпространства (см. п. 1.7).

Дефект (размерность ядра) ограничения формы $\omega$ на $U$ назовем дефектом подпространства $U$ и обозначим через $\operatorname{def} U$. Справедливы следуюшие соотношения:

$$
\begin{gathered}
\operatorname{def} U=\operatorname{def} U^{\perp}=\operatorname{dim}\left(U \cap U^{\perp}\right)=\operatorname{codim}\left(U+U^{\perp}\right), \\
\operatorname{rk} U+\operatorname{def} U=\operatorname{dim} U, \\
\operatorname{cork} U+\operatorname{def} U=\operatorname{codim} U .
\end{gathered}
$$

Пусть задано симплектическое действие группы Ли $G$ на симплектическом многообразии $M$.

ОПРЕДЕЛЕНИЕ 3. Назовем корангом действия $G: M$ коранг касательного пространства орбиты обшего положения. Действия коранга 0 будем назьвать коизотропными.

Коранг действия $G: M$ будем обозначать через $\operatorname{cork}(G, M)$ или cork $M$.

В силу (63) число $\operatorname{cork} M+\operatorname{def} M$ равно коразмерности орбиты общего положения.

2. Коизотропные симплектические действия $G: M$ могут быть охарактеризованы в терминах алгебры Пуассона $\mathscr{P}(M)$.

ПрЕДЛОЖЕнИЕ 5. Симплектическое действие $G: M$ коизотропно тогда и только тогда, когда алгебра $\mathscr{P}(M)^{G}$ коммутативна.

ДокАЗАТЕЛЬСТво. Для точки $p \in M$ обшего положения дифференциалы $G$-инвариантных функций образуют в $T_{p}^{*}(M)$ аннулятор подпространства $\mathfrak{g}_{*}(p) \subset T_{p}(M)$. Соответственно этому их косые градиенты образуют в $T_{p}(M)$ ортогональное дополнение $\mathfrak{g}_{*}(p)^{\perp}$ подпространства $\mathfrak{g}_{*}(p)$. Поэтому коммутативность алгебры $\mathscr{P}(M)^{G}$ равносильна изотропности подпространств $\mathfrak{g}_{*}(p)^{\perp}$ для точек $p$ общего положения, а это как раз и означает коизотропность действия.

3. Рассмотрим частный случай, когда $M=T^{*} X$ и действие $G: M$ индуцировано действием $G: X$.

ОПРеДЕЛЕНИЕ 4. Действие $G: X$ назовем слабо коммутативныцм, если действие $G: T^{*} X$ коизотропно.

В силу предложения 5 это определение согласуется с определением 6 главы I.

ПрЕДЛОЖЕНИЕ 6. Всякое слабо коммутативное действие $G: X$ с редуктивным стабилизатором точки общего положения локально транзитивно. 
ДоказАТельСтво. Нужно доказать, что на $X$ нет непостоянных $G$-инвариантных функций. Предположим, что $\varphi$ - такая функция. Тогда по формуле $(32) v\left(p^{*} \varphi\right)=-d \varphi$. Это инвариантное вертикальное поле, ортогональное орбитам действия $G: T^{*} X$. Ввиду коизотропности последнего оно должно касаться орбит общего положения. Следовательно, для точки $x \in X$ общего положения орбиты общего положения действия $G_{x}: T_{x}^{*} X$ являются цилиндрическими с образующими, параллельньми инвариантному вектору $d_{x} \varphi$. Очевидно, что если группа $G_{x}$ редуктивна, то это невозможно.

4. Предположим теперь, что $G$ - редуктивная комплексная алгебраическая группа, действуюшая на неприводимом гладком комплексном алгебраическом многообразии $X$. В этой ситуации оказывается возможньм выразить коранг и дефект действия $G: T^{*} X$ через известные инварианты действия $G: X$.

Пусть $B$ - борелевская подгруппа и $U$ - содержашаяся в ней максимальная унипотентная подгруппа группы $G$.

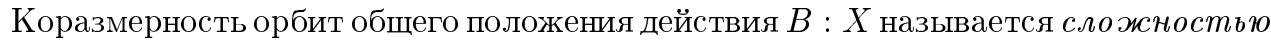
действия $G: X$ и обозначается через $c(X, G)$ или $c(X)$. Действия сложности нуль называются сферическими. В случае, когда $X$ - аффинное многообразие, это согласуется с определением, данньм в п. 3.2 главы I.

Характеры $\lambda$ группы $B$, для которых существует такая рациональная функция $\varphi \in \mathbb{C}(X)$, что $b \varphi=\lambda(b) \varphi$ при $b \in B$, образуют группу, называемую ранговой группой действия $G: X$ и обозначаемую через $\Gamma(X, G)$ или $\Gamma(X)$. Это свободная конечнопорожденная абелева групп. Ее ранг называется рангом действия $G: X$ и обозначается через $r(X, G)$ или $r(x)$.

Эквивалентное определение ранговой групшы состоит в том, что это группа характеров тора $A=(B x) / U$ для точки общего положения $x \in X$. Соответственно этому ранг действия $G: X$ есть разность между сложностью и коразмерностью орбит общего положения действия $U: X$.

Понятие ранга действия является обобщением классического понятия ранга симметрического пространства. А именно, классический ранг симметрического пространства полупростой группы Ли равен рангу его комплексификации в смысле данного вьше определения.

Teopema 2. $\operatorname{cork} T^{*} X=2 c(X), \operatorname{def} T^{*} X=r(X)$.

Эта теорема была фактически доказана в [33]. Ее доказательство в случае, когда многообразие $X$ квазиаффинно, (несколько иное, чем в цитированной статье) будет дано в п. 9. Оно будет опираться на результаты пп. $5-8$.

СЛЕДСТВИЕ 1. Однородное пространство редуктивной группы Ли слабо коммутативно тогда и только тогда, когда оно сферично.

СлЕДСТвИЕ 2. Всякое слабо коммутативное однородное пространство редуктивной группь Ли коммутативно.

5. Действие борелевской подгрупшы на некотором открытом подмножестве многообразия $X$ описьвается так назьваемой теоремой о локальной структуре, доказанной М. Брионом, Д. Луной и Т. Вюстом [34]. (Несколько раньше сходная конструкция рассматривалась $\Phi$. Гроссхансом [35].) 
ТеОРема 3 [34]. Для произвольного действия редуктивной алгебраической группь $G$ на неприводимом алгебраическом многообразии $X$ существует такая параболическая подгруппа $P \subset G$, что некоторое открытое подмножество многообразия $X$ P-эквивариантно изоморфно однородному расслоению $P \underset{L}{*} Y$, где

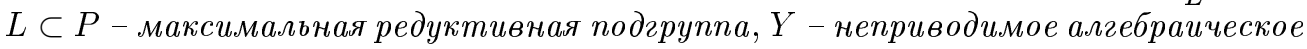
многообразие и коммутант группь L действует на $Y$ тривиально.

Приводимое ниже доказательство принадлежит $\Phi$. Кнопу [36], [37].

ДокаЗАТЕльство. Согласно [38] многообразие $X$ может быть покрыто $G$-инвариантными квазипроективными открытыми подмножествами. Поэтому можно считать, что $X$ - гладкое квазипроективное многообразие. Известно [39], что в этом случае сушествует такое вложение $X \hookrightarrow P V$, что действие $G: X$ индуцируется линейным действием $G: V$.

Пусть $\widehat{X} \subset V$ - конус над $X$. Для любого однородного многочлена $\varphi \in \mathbb{C}[\widehat{X}]$ определим отображение

$$
F_{\varphi}: X_{\varphi} \doteqdot\{x \in X: \varphi(x) \neq 0\} \rightarrow \mathfrak{g}^{*}
$$

по формуле

$$
F_{\varphi}(x)(\xi)=\frac{\xi \cdot \varphi}{\varphi}(x)
$$

Пусть $\varphi-$ собственньй вектор параболической подгруппы $P \subset G$ с весом $\lambda$. Тогда отображение $F_{\varphi} P$-эквивариантно и

$$
F_{\varphi}(x)(\xi)=\lambda(\xi) \text { при } \xi \in \mathfrak{p}
$$

откуда $F_{\varphi}(x) \in \lambda+\mathfrak{p}^{\circ}$, где $\mathfrak{p}^{\circ}-$ аннулятор $\mathfrak{p}$ в $\mathfrak{g}^{*}$.

Выбрав инвариантное скалярное умножение в $\mathfrak{g}$, условимся отождествлять $\mathfrak{g}^{*} \mathrm{c} \mathfrak{g}$. Пусть $U_{P}-$ унипотентньй радикал группы $P$. Тогда $\mathfrak{p}^{\circ}=\mathfrak{p}^{\perp}=\mathfrak{u}_{P}$. Таким образом, мы получим $P$-эквивариантное отображение

$$
F_{\varphi}: X_{\varphi} \rightarrow \lambda+\mathfrak{u}_{P}
$$

Будем считать, что

$$
P=\{g \in G: g \varphi \in\langle\varphi\rangle\} .
$$

Тогда оператор $\operatorname{ad}(\lambda)$ невырожден (и, более того, имеет только положительные собственные значения) на $\mathfrak{u}_{P}$, откуда следует, что $\lambda+\mathfrak{u}_{P}$ есть одна $P$-орбита, причем стабилизаторы ее точек - это максимальные редуктивные подгрупшы группы $P$.

Пусть $L \subset P$ - стабилизатор точки $\lambda$ и $Y=F_{\varphi}^{-1}(\lambda)$. Тогда отображение

$$
P \times Y \rightarrow X_{\varphi}, \quad(p, y) \mapsto p y
$$

индуцирует $P$-эквивариантньй биективный морфизм

$$
P \underset{L}{*} Y \rightarrow X_{\varphi}
$$

которьй ввиду гладкости многообразия $X_{\varphi}$ является изоморфизмом. Так как база расслоения $P \underset{L}{*} Y$ односвязна, то многообразие $Y$ неприводимо. 
Отметим, что если коммутант групшы $G$ действует на $X$ нетривиально, то функция $\varphi$ может быть выбрана так, что $P \neq G$. Поэтому если коммутант группы $L$ действует на $Y$ нетривиально, то, применив доказанное вьше к действию $L: Y$, мы можем уменьшить подгруппу $P$. Продолжая так дальше, мы в конце конщов добьемся нужного результата.

Пусть $P \subset G$ - параболическая подгруппа, удовлетворяюшая требованиям теоремы, и $U_{P}$ - ее унипотентньй радикал. Обозначим через $L_{0}$ ядро неэффективности действия $L: Y$ и положим

$$
S=U_{P} \lambda L_{0} \subset P .
$$

Следуюшее предложение показьвает, в частности, что подгрупшы $P$ и $S$ с точностью до сопряженности однозначно определяются действием $G: X$.

ПРЕДЛОЖЕНИЕ 7 [40], [36]. Подгруппа $P$ (соответственно подгруппа $S)$ совпадает со стабилизатором B-орбиты (соответственно U-орбиты) любой точки $x$ (соответственно точки $x$ общего положения) из открытого подмножества, удовлетворяющего требованиям теоремы.

ДокаЗАтЕльСтво. Очевидно, что $P x=B x, S x=U x$ и $G_{x} \cap U_{P}=\{e\}$.

Пусть $\widetilde{S} \supset S-$ стабилизатор орбиты $U x$ и $\widetilde{U}_{P} \subset U_{P}-$ его унипотентный радикал. Из разложения $\widetilde{S}=\widetilde{S}_{x} \cdot U_{P}$ следует, что многообразие $\left(\widetilde{S} / \widetilde{U}_{P}\right) /\left(\widetilde{U} / \widetilde{U}_{P}\right)=\widetilde{S} / U_{P} \simeq \widetilde{S}_{x}$ аффинно и, значит, подгрупа $U_{P} / \widetilde{U}_{P} \subset \widetilde{S} / \widetilde{U}_{P}$ редуктивна. Это возможно, только если $U_{P}=\widetilde{U}_{P}$; но тогда $\widetilde{S} \subset N\left(U_{P}\right)=P$ и, следовательно, для точки $x$ общего положения $\widetilde{S}=S$.

Стабилизатор орбиты $B x$ есть произведение подгруппы $B$ и стабилизатора орбиты $U x$ и, следовательно, совпадает с $P$.

Факторгруппа $A=L / L_{0}$ есть тор, совпадающий с тором $A$, определенным в п. 4. В силу теоремы 90 Гильберта действие $A: Y$ допускает рациональное сечение. Это означает, что некоторое инвариантное открытое подмножество в $Y$ можно представить как $A \times C$, где $C$ - алгебраическое многообразие, на котором группа $A$ действует тривиально, а действие $A: A$ определяется как групповое умножение. Уменњшив, если нужно, многообразие $Y$, будем в дальнейшем считать, что оно само представляется в указанном виде.

Очевидно, что

$$
c(X)=\operatorname{dim} C, \quad r(X)=\operatorname{dim} A .
$$

Более того, ранговая группа $\Gamma(X)$ есть группа характеров тора $A$ (вложенная естественным образом в группу характеров групшы $P$ ).

Учитывая, что группа $U_{P}$ действует просто транзитивно на $P / L$, получаем, что некоторое $P$-инвариантное открытое подмножество $X_{0} \subset X$ представляется в виде

$$
X_{0}=U_{P} \times Y=U_{P} \times A \times C,
$$

причем действие группы $P=U_{P} \lambda L$ на $X_{0}$ описьвается формулами

$$
\begin{gathered}
v(u, a, c)=(v u, a, c) \quad\left(v \in U_{P}\right), \\
l(u, a, c)=\left(l u l^{-1}, \pi(l) a, c\right) \quad(l \in L),
\end{gathered}
$$

где $\pi: L \rightarrow L / L_{0}=A-$ канонический гомоморфизм. 
6. В случае, когда многообразие $X$ квазиаффинно, теорема 3 допускает важные уточнения.

Обозначим через a ортогональное дополнение к идеалу $\mathfrak{l}_{0}$ в алгебре l. Это диагонализируемая подалгебра, которая естественным образом отождествляется с касательной алгеброй тора $A$. Ясно, что $\mathfrak{l} \subset \mathfrak{z}(\mathfrak{a})$.

ПРЕДЛОЖЕНИЕ 8. Если многообразие $X$ квазиаффинно, то

1) подгруппа $P$ (соответственно $S$ ) совпадает с подгруппой $P^{\prime}$ (соответственно $\left.S^{\prime}\right)$ всех әлементов группь $G$, для которых всякий стариий вектор представления $G: \mathbb{C}[X]$ является собственным (соответственно неподвижным м) вектором;

2) подпространство а совпадает с линейной оболочкой всех старших весов представления $G: \mathbb{C}[X]$;

3) $\mathfrak{l}=\mathfrak{z}(\mathfrak{a})$.

ДокаЗАТЕльство. Так как $U$-орбиты общего положения совпадают с $S$-орбитами, то всякий $U$-инвариант является $S$-инвариантом и, следовательно, $S \subset S^{\prime}$.

По теореме Розенлихта $U$-орбиты общего положения разделяются рациональными $U$-инвариантами. Если многообразие $U$ квазиаффинно, то всякий рациональньй $U$-инвариант является отношением регулярных $U$-инвариантов и, следовательно, $U$-орбиты общего положения разделяются регулярными $U$-инвариантами. Кроме того, в этом случае все $U$-орбиты замкнуты. Отсюда следует, что $U$-орбиты общего положения совпадают с $S^{\prime}$-орбитами и, значит, $S=S^{\prime}$.

Так как подгруппа $S$ (соответственно $S^{\prime}$ ) содержит максимальную полупростую подгруппу подгруппы $P$ (соответственно $\left.P^{\prime}\right)$, то из равенства $S=S^{\prime}$ следует, что $P=P^{\prime}$.

Остальные утверждения легко вьводятся из доказанного.

7. Следуя И. М. Гельфанду и М.И. Граеву [41], назовем орисферами для действия $G: X$ орбиты максимальных унипотентных подгрупп группы $G$ или, что эквивалентно, образы при действии группы $G$ орбит фиксированной максимальной унипотентной подгрупшы $U$. Групша $G$ естественным образом действует на множестве всех орисфер.

Обозначим через Hor $X$ множество орисфер, $G$-эквивалентных $U$-орбитам точек построенного выше открытого подмножества $X_{0} \subset X$. Множество $U$-орбит в $X_{0}$ параметризуется многообразием $Y=A \times C$ и инвариантно относительно групшы $P$; при этом действие группы $P$ на этом множестве сводится к действию тора $P / S=A$.

Лемма 2. Если $g U x=U y\left(x, y \in X_{0}\right)$, mo $g \in P$.

ДокАЗАТЕльство. Из предложения 7 следует, что $g \in N(S)$; но так как $P$ - наименьшая параболическая подгруппа, содержашая $S$, то $N(S) \subset N(P)=P$ (и, значит, $N(S)=P)$.

Отсюда следует, что множество Hor $X$ представляется в виде

$$
\text { Hor } X=G \underset{P}{*} Y=G / S \times C
$$

и, тем самым, наделяется структурой алгебраического многообразия, относительно которой действие $G$ : Hor $X$ является алгебраическим. Построенное таким образом алгебраическое многообразие будем назьвать многообразием орисфер для действия $G: X$. 
Tак как $\operatorname{dim} G / P=\operatorname{dim} U_{P}$, то

$$
\operatorname{dim} \operatorname{Hor} X=\operatorname{dim} G / P+\operatorname{dim} Y=\operatorname{dim} U_{P}+\operatorname{dim} Y=\operatorname{dim} X \text {. }
$$

Следует заметить, что в определении подмножества $X_{0}$, а значит, и многообразия Hor $X$ имеется некоторьй произвол. Однако он не будет играть роли в последующем изложении.

8. Займемся теперь многообразием $T^{*} X$.

Напомним некоторые определения. Подмногообразие $N$ симплектического многообразия $M$ назьвается изотропным, если его касательное пространство в каждой точке изотропно. Изотропное подмногообразие максимальной возможной размерности (равной $\left.\frac{1}{2} \operatorname{dim} M\right)$ назьвается лагранжевылм.

Для любого подмногообразия $Z \subset X$ определим его конормальное расслоение

$$
N^{*} Z=\left\{\alpha \in T_{z}^{*}(X): z \in Z, \alpha=0 \text { на } T_{z}(Z)\right\} \subset T^{*} X
$$

Из формул (34)-(36) следует, что $N^{*} Z$ - изотропное подмногообразие в $T^{*} X$. Так как

$$
\operatorname{dim} N^{*} Z=\operatorname{dim} X=\frac{1}{2} \operatorname{dim} T^{*} X,
$$

то $N^{*} Z$ - лагранжево подмногообразие.

Рассмотрим, в частности, конормальные расслоения орисфер. Заметим, что многообразие $T^{*} X$ ими покрывается. В самом деле, для любой линейной формы $\alpha \in T_{x}^{*}(X)$ ее образ при отображении моментов $\Phi: T^{*} X \rightarrow \mathfrak{g}^{*}=\mathfrak{g}$, как и любой элемент алгебры $\mathfrak{g}$, содержится в некоторой борелевской подалгебре $\mathfrak{b}_{1}$. Ортогональное дополнение к подалгебре $\mathfrak{b}_{1}$ есть ее унипотентньй радикал $\mathfrak{u}_{1}$, и из формулы $(52)$ для отображения моментов следует, что $\alpha \in N^{*} U_{1} x$.

Если, однако, ограничиться орисферами из определенного вьше множества Hor $X$, то может случиться, что их конормальные расслоения будут покрьвать лишш незначительную часть многообразия $T^{*} X$. Так, например, в случае проективной прямой их объединение есть нулевое сечение расслоения $T^{*} X$. Мы покажем, что для аффинных многообразий такой неприятности случиться не может. В обшем случае указанную трудность, вероятно, можно обойти, надлежащим образом изменив определение множества Hor $X$. Так, в случае проективной прямой “правильными” орисферами следует считать не дополнения к точкам, а точки (которые также являются орбитами максимальных унипотентных подгрупп).

Построим вспомогательное многообразие, являющееся дизъюнктивньм объединением конормальных расслоений орисфер из множества Hor $X$.

В соответствии с (65) получаем следуюшее представление для конормального расслоения $U$-орбиты точки $y \in Y \subset X_{0}$ :

$$
N^{*}(U y)=U_{P} \times T_{y}^{*}(Y) \subset T^{*} X_{0}
$$

Рассмотрим многообразие

$$
H T^{*} X=G \underset{P}{*}\left(U_{P} \times T^{*} Y\right)=G \underset{S}{*}\left(U_{P} \times \mathfrak{a}\right) \times T^{*} C\left(=G / L_{0} \times \mathfrak{a} \times T^{*} C\right) .
$$


Оно является расслоением над многообразием орисфер Hor $X$, слоями которого служат конормальные расслоения соответствуюших орисфер. Назовем это многообразие орисферическим кокасательным расслоением многообразия $X$.

Заметим, что

$$
\operatorname{dim} H T^{*} X=\operatorname{dim} \operatorname{Hor} X+\operatorname{dim} X=\operatorname{dim} T^{*} X .
$$

Имеется $G$-эквивариантньй морфизм

$$
p: H T^{*} X \rightarrow T^{*} X,
$$

индуцированньй вложением

$$
U_{P} \times T^{*} Y \hookrightarrow T^{*} X
$$

Теорема 4 [36]. Если многообразие $X$ квазиаффинно, то морфизм р доминантен и, значит, является рачиональным накрытием.

(Доминантное рациональное отображение $p: M \rightarrow N$ назьвается рациональным накрытием, если поле $\mathbb{C}(M)$ является конечным расширением поля $p^{*}(\mathbb{C}(M))$ или, что равносильно, если $\operatorname{dim} M=\operatorname{dim} N$. Всякое рациональное накрытие является накрытием в обычном смысле слова над некоторым открытьм подмножеством многообразия $N$.)

ДоКАЗАТЕЛЬСТВо. Положим

$$
M=T^{*} X_{0}, \quad N=U_{P} \times T^{*} Y \subset M .
$$

Пусть $U_{P}^{-}$- унипотентньй радикал параболической подгруппы, противоположной $P$, так что

$$
\mathfrak{g}=\mathfrak{u}_{P}^{-} \oplus \mathfrak{l} \oplus \mathfrak{u}_{P}
$$

Так как $P N=N$ и

$$
\operatorname{dim} M-\operatorname{dim} N=\operatorname{dim} U_{P}=\operatorname{dim} U_{P}^{-},
$$

то доминантность морфизма $p$ равносильна существованию такой точки $\alpha \in$ $T_{y}^{*}(Y) \subset N(y \in Y)$, что

$$
T_{\alpha}(M)=T_{\alpha}(N) \oplus \mathfrak{u}_{P}^{-} \alpha .
$$

Заметим, что подмногообразие $N$ коизотропно, причем его слоями вырождения служат $U_{P}$-орбиты (они же $U$-орбиты). Поэтому условие $(72)$ равносильно невырожденности спаривания

$$
\mathfrak{u}_{P} \times \mathfrak{u}_{P}^{-} \rightarrow \mathbb{C}, \quad(\xi, \eta) \mapsto \omega(\xi \alpha, \eta \alpha)=\alpha([\xi, \eta] y),
$$

где $\omega$ - каноническая 2-форма на $M=T^{*} X$ (см. формулу (36)).

Пусть $\varphi \in \mathbb{C}[X]$ - старший вектор представления $G: \mathbb{C}[X]$, вес которого ортогонален минимальному возможному числу корней, т.е. только корням подгруппы $L$. Возьмем в качестве $\alpha$ дифференциал функции $\varphi$ в любой точке $y \in Y$, в которой $\varphi$ не обрашается в нуль. Тогда

$$
\alpha([\xi, \eta] y)=-([\xi, \eta] \cdot \varphi)(y) .
$$

Заметим, что если $\zeta \in \mathfrak{s}$, то $\zeta \cdot \varphi=0$. Поэтому если $\xi=e_{\alpha} \in \mathfrak{u}_{P}, \eta=e_{-\beta} \in \mathfrak{u}_{P}^{-}-$ корневые векторы, то $[\xi, \eta] \cdot \varphi=0$ при $\alpha>\beta$ (в смысле лексикографического упорядочения) и $[\xi, \eta] \cdot \varphi=c \varphi\left(c \in \mathbb{C}^{*}\right)$ при $\alpha=\beta$. Таким образом, в базисах пространств $\mathfrak{u}_{P}$ и $\mathfrak{u}_{P}^{-}$, составленных из корневых векторов, матрица спаривания (73) треугольная с ненулевыми диагональными элементами, и, значит, это спаривание невырожденно. 
СлЕДСТВИЕ. Если $X$ квазиаффинно, то стабилизаторы точек общего положения для действия $G: T^{*} X$ сопряжень конечному расширению подгруппы $L_{0}$.

ДокАЗАТЕЛЬСтво. Из теоремы и определения многообразия $H T^{*} X$ следует, что искомые стабилизаторы сопряжены конечному расширению стабилизатора точки общего положения для действия $L: T^{*} Y$, которьй, очевидно, равен $L_{0}$.

9. В предположении теоремы 4 найдем замыкание образа отображения моментов для действия $G: T^{*} X$. Из теоремы следует, что

$$
\overline{\Phi\left(T^{*} X\right)}=\overline{\operatorname{Ad}(G) \Phi\left(T^{*} Y\right)} .
$$

Так как подгруппа $S$ сохраняет $U$-орбиты, то (при отождествлении $\mathfrak{g}^{*} \mathrm{c} \mathfrak{g}$ )

$$
\Phi\left(T^{*} Y\right) \subset \mathfrak{s}^{\perp}=\mathfrak{u}_{P} \oslash \mathfrak{a} .
$$

Рассматривая действие группы $A$, находим, что множество $\Phi\left(T^{*} Y\right)$ проектируется на всю подалгебру $\mathfrak{a}$.

Из утверждения 3 ) предложения 8 следует, что алгебра $\mathfrak{a}$ не имеет нулевых весов в $\mathfrak{u}_{P}$ и, значит,

$$
\mathfrak{a}^{\text {reg }} \doteqdot\left\{\xi \in \mathfrak{a}:\left[\xi, \mathfrak{u}_{P}\right]=\mathfrak{u}_{P}\right\}
$$

есть непустое открытое подмножество в $\mathfrak{a}$. Для каждого элемента $\xi \in \mathfrak{a}^{\text {reg }}$ его $U_{P}$-орбита есть плоскость $\xi+\mathfrak{u}_{P}$. Следовательно,

$$
\overline{\operatorname{Ad}\left(U_{\mathfrak{p}}\right) \Phi\left(T^{*} Y\right)}=\mathfrak{u}_{P} \ni \mathfrak{a}=\overline{\operatorname{Ad}\left(U_{P}\right) \mathfrak{a}}
$$

Из (74) и (75) получаем окончательно, что

$$
\overline{\Phi\left(T^{*} X\right)}=\overline{\operatorname{Ad}(G) \mathfrak{a}}
$$

В частности, элементы обшего положения в $\Phi\left(T^{*} X\right)$ полупросты, т.е. действие $G: T^{*} X$ симплектически стабильно. Тем самьм доказана теорема 1.

Докажем теперь теорему 2 для квазиаффинного многообразия $X$.

Из (76) следует, что $\operatorname{dim} \pi_{G}\left(\Phi\left(T^{*} X\right)\right)=\operatorname{dim} \mathfrak{a}$. Это означает, что $\operatorname{def} T^{*} X=r(X)$. Далее, в силу следствия теоремы 4 коразмерность орбиты общего положения для действия $G: T^{*} X$ равна

$$
\operatorname{dim} T^{*} X-\operatorname{dim} G / L_{0}=2\left(\operatorname{dim} X-\operatorname{dim} U_{P}\right)-\operatorname{dim} \mathfrak{a},
$$

откуда

$$
\operatorname{cork} T^{*} X=2\left(\operatorname{dim} X-\operatorname{dim} U_{P}-\operatorname{dim} A\right)=2 \operatorname{dim} C=2 c(X),
$$

что и требовалось доказать.

10. Для квазиаффинного многообразия $X$ построенное выше рациональное накрытие $p: H T^{*} X \rightarrow T^{*} X$ позволяет перенести каноническую симплектическую структуру многообразия $T^{*} X$ на некоторое открытое подмножество многообразия $H T^{*} X$. Пересечения конормальных расслоений орисфер с этим подможеством образуют лагранжево расслоение с базой Hor $X$. Согласно общей теории (см., например, [29]) гамильтоновы векторные поля, определяемые функциями на базе лагранжева расслоения, т.е. в данном случае на Hor $X$, касаются слоев и в каждом слое определяют 
каноническую локально афффинную структуру, относительно которой они являются постоянными; при этом ассоциированное векторное пространство есть кокасательное пространство базы. В этом смысле многообразие $H T^{*} X$ похоже на кокасательное расслоение $T^{*}$ Hor $X$ многообразия орисфер.

С другой стороны, имеется $G$-эквивариантньй бирациональный морфиизм

$$
f: H T^{*} X \rightarrow T^{*} \operatorname{Hor} X
$$

определяемый следуюшим образом.

Из представления многообразия Hor $X$ в виде (68) получаем:

$$
T^{*} \operatorname{Hor} X=T^{*}(G / S) \times T^{*} C=\underset{S}{*}(\mathfrak{g} / \mathfrak{s})^{*} \times T^{*} C
$$

При отождествлении $\mathfrak{g}^{*}$ с $\mathfrak{g}$ пространство $(\mathfrak{g} / \mathfrak{s})^{*}$ отождествляется с подпространством

$$
\mathfrak{s}^{\perp}=\mathfrak{u}_{P} \oslash \mathfrak{a} \subset \mathfrak{g} .
$$

Таким образом, многообразие $T^{*}$ Hor $X$ представляется в виде

$$
T^{*} \operatorname{Hor} X=G \underset{S}{*}\left(\mathfrak{u}_{P} \oslash \mathfrak{a}\right) \times T^{*} C .
$$

Рассмотрим $S$-эквивариантный морфизм

$$
f_{0}: U_{P} \times \mathfrak{a} \rightarrow \mathfrak{u}_{P} \oplus \mathfrak{a}, \quad(u, \xi) \mapsto \operatorname{Ad}(u) \xi
$$

Из того, что $\mathfrak{a}$ не имеет нулевых весов в $\mathfrak{u}_{P}$ (утверждение 3 ) предложения 8), следует, что морфизм $f_{0}$ бирашионален. Используя представления (71) и (78) многообразий $H T^{*} X$ и $T^{*}$ Hor $X$, мы можем теперь определить искомый морфизм $f$ по формуле

$$
f((g z, \gamma))=\left(g f_{0}(z), \gamma\right) \quad\left(g \in G, z \in U_{P} \times \mathfrak{a}, \gamma \in T^{*} C\right)
$$

Нетрудно видеть, что морфизм $f$ переводит конормальные расслоения орбит в соответствующие кокасательные пространства многообразия Hor $X$, т.е. имеет место коммутативная диаграмма

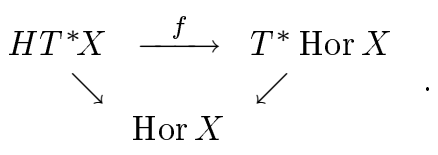

Вообще говоря, морфизм $f$ не является симплектическим (ни при каком выборе $A$-сечения $C$ в $Y$ ). Однако можно показать, что для (комплексных) симметрических пространств он является симплектическим. Таким образом, для всякого симметрического пространства $X$ имеется $G$-эквивариантное симплектическое рациональное накрытие

$$
T^{*} \operatorname{Hor} X \rightarrow T^{*} X
$$




\section{§4. Структура слабо коммутативных однородных пространств}

1. Напомним, что однородное пространство $X=G / K$ мы называем слабо коммутативным, если действие $G: T^{*} X$ коизотропно. Очевидно, что свойство слабой коммутативности является локальным, т.е. зависит только от пары $(\mathfrak{g}, \mathfrak{k})$.

Пространство $T_{o}(X)$ канонически отождествляется с $\mathfrak{g} / \mathfrak{k}$. Соответственно этому пространство $T_{o}^{*}(X)$ отождествляется с $(\mathfrak{g} / \mathfrak{k})^{*}=\mathfrak{k}^{\circ} \subset \mathfrak{g}^{*}$. Действие $K: T_{o}^{*}(X)$ индуцируется коприсоединенным представлением $\mathrm{Ad}^{*}$ групшы $G$.

ПрЕДЛОЖЕнИЕ 9. Орбита точки $\alpha \in T_{o}^{*}(X)$ в $T^{*} X$ коизотропна тогда и только тогда, когда выполнено любое из следующих эквивалентных условий:

(K1) $\operatorname{dim} \operatorname{Ad}^{*}(G) \alpha=2 \operatorname{dim} \operatorname{Ad}^{*}(K) \alpha$;

(K2) $\operatorname{ad}^{*}(\mathfrak{g}) \alpha \cap \mathfrak{k}^{\circ}=\operatorname{ad}^{*}(\mathfrak{k}) \alpha$;

(K3) $\alpha\left(\left[\left(\operatorname{ad}^{*}(\mathfrak{k}) \alpha\right)^{\circ},\left(\operatorname{ad}^{*}(\mathfrak{k}) \alpha\right)^{\circ}\right]\right)=0$.

ДоКАЗАТЕЛЬСТвО. Коразмерность орбиты точки $\alpha$ в $T^{*} X$ равна $\operatorname{dim} G / K-$ $\operatorname{dim} \operatorname{Ad}^{*}(K) \alpha$. С другой стороны, ее деф̆ект равен $\operatorname{dim} G_{\alpha}-\operatorname{dim} K_{\alpha}$. Следовательно, ее коранг равен

$$
\left(\operatorname{dim} G-\operatorname{dim} G_{\alpha}\right)-2\left(\operatorname{dim} K-\operatorname{dim} K_{\alpha}\right)=\operatorname{dim} \operatorname{Ad}^{*}(G) \alpha-2 \operatorname{dim} \operatorname{Ad}^{*}(K) \alpha .
$$

В частности, орбита точки $\alpha$ коизотропна тогда и только тогда, когда выполнено условие (K1) или эквивалентное ему условие

$$
\operatorname{dim} \operatorname{ad}^{*}(\mathfrak{g}) \alpha=2 \operatorname{dim} \operatorname{ad}^{*}(\mathfrak{k}) \alpha .
$$

Так как $\alpha \in \mathfrak{k}^{\circ}$, то подпространство аd* ${ }^{*}(\mathfrak{k}) \alpha$ изотропно в $\mathrm{ad}^{*}(\mathfrak{g}) \alpha$ в смысле формы Костанта-Кириллова (40). Поэтому условие (80) равносильно тому, что оно является максимальньм изотропным подпространством в $\mathrm{ad}^{*}(\mathfrak{g}) \alpha$, т.е. совпадает со своим ортогональным дополнением. Но его ортогональное дополнение - это подпространство $\operatorname{ad}^{*}(\mathfrak{g}) \alpha \cap \mathfrak{k}^{\circ}$. Таким образом, условие (80) равносильно условию (K2).

Легко видеть, что $\operatorname{ad}^{*}(\xi) \alpha \in \mathfrak{k}^{\circ}$ тогда и только тогда, когда $\xi \in\left(\operatorname{ad}^{*}(\mathfrak{k}) \alpha\right)^{\circ}$. Поэтому условие (К3) означает, что подпространство $\mathrm{ad}^{*}(\mathfrak{g}) \alpha \cap \mathfrak{k}^{\circ}$ изотропно в смысле формы Костанта-Кириллова, что также равносильно (80).

СлеДСТвИЕ. Однородное пространство $X=G / K$ слабо коммутативно тогда и только тогда, когда для точки а общего положения в $T_{o}^{*}(X)$ выполнено любое из эквивалентных условий (K1)-(K3).

Интересно отметить, что условие (K2) для точки общего положения есть не что иное, как известное в теории инвариантов (в более общей ситуации) условие Ричардсона [2]. Из него следует, что каждая орбита общего положения групшы $\operatorname{Ad}^{*}(G)$ пересекает подпространство $\mathfrak{k}^{\circ} \subset \mathfrak{g}^{*}$ не более чем по конечному числу орбит групш $\operatorname{Ad}^{*}(K)$, каждая из которых замкнута (и открыта) в этом пересечении.

2. Начиная с этого момента, будем предполагать, что подгруппа $K$ компактна. В этом случае орбиты действия $K:(\mathfrak{g} / \mathfrak{k})^{*}$ (как и орбиты любой компактной линейной групшы) разделяются полиномиальньми инвариантами. Алгебру полиномиальных инвариантов этого действия обозначим через $\mathbb{R}\left[(\mathfrak{g} / \mathfrak{k})^{*}\right]^{K}$. 
ПРЕДЛОЖЕНИЕ 10. Однородное пространство $X=G / K$ слабо коммутативно тогда и только тогда, когда

$$
\alpha\left(\left[d_{\alpha} \varphi, d_{\alpha} \psi\right]\right)=0
$$

для любых инвариантов $\varphi, \psi \in \mathbb{R}\left[(\mathfrak{g} / \mathfrak{k})^{*}\right]^{K}$ и любой точки $\alpha \in T_{o}^{*}(X)=\mathfrak{k}^{\circ}$.

(Здесь предполагается, что элементы $d_{\alpha} \varphi, d_{\alpha} \psi \in \mathfrak{g} / \mathfrak{k}$ каким-то образом подняты в $\mathfrak{g}$; вьполнение равенства (81) не зависит от способа этого поднятия, так как дифференщиалы инвариантов обращаются в нуль на касательных пространствах орбит.)

ДокАЗАТЕльство. В точке $\alpha$ общего положения дифференциалы инвариантов образуют конормальное пространство орбиты $\mathrm{Ad}^{*}(K) \alpha$, т.е. пространство $\left(\mathrm{ad}^{*}(\mathfrak{k}) \alpha\right)^{\circ}$. Поэтому условие (81) для точек общего положения совпадает с условием (К3). Но по соображениям непрерьвности из его вьполнения в точках общего положения следует его выполнение всюду.

СлЕДСТВИЕ 1. Если пространство $G / K$ слабо коммутативно, то для любой нормальной подгруппь Ли $N$ группь $G$ пространство

$$
G / N K=(G / N) /(K /(N \cap K))
$$

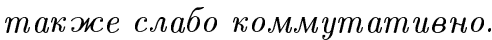

ДоКАЗАТЕЛЬСТво следует из того, что пространство $T_{o}^{*}(G / N K)$ есть $K$-инвариантное подпространство в $T_{o}^{*}(G / K)$ и алгебра $\mathbb{R}\left[T_{o}^{*}(G / N K)\right]^{N K}=\mathbb{R}\left[T_{o}^{*}(G / N K)\right]^{K}$ есть ограничение алгебры $\mathbb{R}\left[T_{o}^{*}(G / K)\right]^{K}$ на это подпространство.

СлЕДСТВИЕ 2. Если пространство $G / K$ слабо коммутативно, то для любой компактной подгруппы $L \subset G$, содержащей $K$, пространство $G / L$ также слабо коммутативно.

ДокАЗАТЕльСТво аналогично доказательству следствия 1.

СлЕДСТВИЕ 3. Если пространство $G / K$ слабо коммутативно, то для любой подгруппы Ли $L \subset G$, содержащей $K$, пространство $L / K$ также слабо коммутативно.

ДокАЗАТЕльство следует из того, что пространство $T_{o}^{*}(L / K)$ есть факторпространство пространства $T_{o}^{*}(G / K)$ по $K$-инвариантному подпространству и алгебра $\mathbb{R}\left[T_{o}^{*}(L / K)\right]^{K}$ есть подалгебра алгебры $\mathbb{R}\left[T_{o}^{*}(G / K)\right]^{K}$.

3. Пусть $X=G / K$ - слабо коммутивное пространство. Выберем в $\mathfrak{g}$ какое-нибудь $K$-инвариантное скалярное умножение и обозначим через $\mathfrak{m}$ ортогональное дополнение в $\mathfrak{k}$. Тогда пространства $\mathfrak{g} / \mathfrak{k}$ и $(\mathfrak{g} / \mathfrak{k})^{*}$ можно будет естественным образом отождествить с $\mathfrak{m}$. Условие (81) при этом перепишется в виде

$$
\left(a,\left[\operatorname{grad}_{a} \varphi, \operatorname{grad}_{a} \psi\right]\right)=0 \quad\left(a \in \mathfrak{m}, \varphi, \psi \in \mathbb{R}[\mathfrak{m}]^{K}\right)
$$

Следуюшее две леммы будут играть ключевую роль в нашем описании структуры слабо коммутативных однородных пространств. 
Лемма 3. Пусть $\mathfrak{a}, \mathfrak{b} \subset \mathfrak{g}-$ такие $K$-инвариантные подпространства, что подпространства $\mathfrak{k}, \mathfrak{a}, \mathfrak{b}$ линейно независимы. Тогда

$$
[\mathfrak{a}, \mathfrak{b}] \subset \mathfrak{k}+\mathfrak{a}+\mathfrak{b} .
$$

Кроме того, если К-инвариантное скалярное умножение в $\mathfrak{g}$ выбрано так, что подпространства $\mathfrak{k}, \mathfrak{a}, \mathfrak{b}$ ортогональны, то

$$
([a, b], a)=0
$$

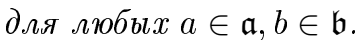

ДоКАЗАТЕЛЬСтво. Будем считать подпространства $\mathfrak{k}, \mathfrak{a}, \mathfrak{b}$ ортогональными (что всегда можно сделать) и обозначим через с ортогональное дополнение $\mathfrak{k}+\mathfrak{a}+\mathfrak{b}$. Пусть $P$ и $Q$ - ортогональные проекторы на $\mathfrak{a}$ и $\mathfrak{b}$ соответственно. Записывая условие $(82)$ для дифференциалов инвариантов $\varphi(x)=(P x, x)$ и $\psi(x)=(Q x, x)$ в точке $\lambda a+\mu b+c$ $(\lambda, \mu \in \mathbb{R}, a \in \mathfrak{a}, b \in \mathfrak{b}, c \in \mathfrak{c})$, получаем, что

$$
([a, b], \lambda a+\mu b+c)=0,
$$

откуда все следует.

Лемма 4. Пусть $L \subset G-$ связная подгруппа Ли, содержащая $K, u \mathfrak{p} \subset \mathfrak{g}-$ L-инвариантное подпространство, дополнительное $\kappa \mathfrak{l}$. Тогда

$$
\mathbb{R}[\mathfrak{p}]^{L}=\mathbb{R}[\mathfrak{p}]^{K} .
$$

ДоКАЗАТЕльСТВо. Можно считать, что $\mathfrak{p} \perp \mathfrak{l}$. Пусть $\mathfrak{q}-$ ортогональное дополне-

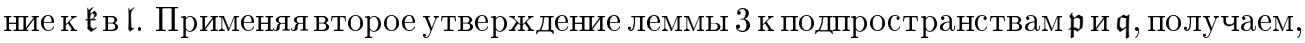
что $\mathfrak{l}$ действует на $\mathfrak{p}$ кососимметрическими операторами.

Далее, пусть $Q$ - ортогональный проектор на q. Записывая условие (82) для дифоференциалов инвариантов $\varphi \in \mathbb{R}[\mathfrak{p}]^{K} \subset \mathbb{R}[\mathfrak{m}]^{K}$ и $\psi(x)=(Q x, x)$ в точке $a+b(a \in \mathfrak{p}$, $b \in \mathfrak{q})$, получаем:

$$
\left(\left[\operatorname{grad}_{a} \varphi, b\right], a+b\right)=0 .
$$

Учитьвая, что $\operatorname{grad}_{a} \varphi \in \mathfrak{p}$ и $\operatorname{ad}(b)$ по доказанному вьше действует на $\mathfrak{p}$ как кососимметрический оператор, это равенство можно переписать в виде

$$
\left(\partial_{[a, b]} \varphi\right)(a)=0 .
$$

Это означает, что полином $\varphi \in \mathbb{R}[\mathfrak{p}]$ постоянен на орбитах групшы $L$, т.е. $\varphi \in \mathbb{R}[\mathfrak{p}]^{L}$.

4. Следуюшая теорема описывает структуру слабо коммутативных однородных пространств нередуктивных групп Ли.

Теорема 5. Пусть $X=G / K$ - односвязное слабо коммутативное риманово однородное пространство связной группь Ли $G$. Тогда $G=N 入 L$, где

1) $N$ - не более чем 2-ступенно нильпотентная односвязная группа Ли;

2) $L$ - связная редуктивная подгруппа Ли, содержащая $K$;

3) $\mathbb{R}[\mathfrak{n}]^{L}=\mathbb{R}[\mathfrak{n}]^{K}$.

В частности, из 3$)$ следует, что группа $\operatorname{Ad}_{\mathfrak{n}}(L)$ содержится в ортогональной группе $\mathrm{O}(\mathfrak{n})$ пространства $\mathfrak{n}$. 
ДоКАЗАТЕльство. Пусть $\mathfrak{n}$ - наибольший нильпотентный идеал алгебры $\mathfrak{g}$. Легко видеть, что $\mathfrak{n} \cap \mathfrak{k}=0$. Пусть $\mathfrak{a}-$ ортогональное дополнение к $[\mathfrak{n}, \mathfrak{n}]$ в $\mathfrak{n}$ и $\mathfrak{b}-$ ортогональное дополнение к $[[\mathfrak{n}, \mathfrak{n}], \mathfrak{n}]$ в $[\mathfrak{n}, \mathfrak{n}]$. Очевидно, что это $K$-инвариантные подпространства. Применяя к ним лемму 3 , получаем, что $[\mathfrak{a}, \mathfrak{b}]=0$. Это означает, что $[[\mathfrak{n}, \mathfrak{n}], \mathfrak{n}]=0$.

Пусть, далее, $\mathfrak{r}$ - радикал и $\mathfrak{s}$ - максимальная полупростая подалгебра алгебры $\mathfrak{g}$. Подалгебру $\mathfrak{s}$ можно выбрать $K$-инвариантной. Тогда $\mathfrak{k}+\mathfrak{s}$ будет редуктивной подалгеброй. Легко видеть, что $\mathfrak{n} \cap(\mathfrak{k}+\mathfrak{s})=0$. Поэтому существует такое $(\mathfrak{k}+\mathfrak{s})$-инвариантное подпространство $\mathfrak{c} \subset \mathfrak{r}$, что $\mathfrak{n} \cap \mathfrak{c}=0$ и $\mathfrak{n}+\mathfrak{c}-$ подпространство, дополнительное к $\mathfrak{k}+\mathfrak{s}$. Так как $[\mathfrak{g}, \mathfrak{r}] \subset \mathfrak{n}[19]$, то $[\mathfrak{k}+\mathfrak{s}, \mathfrak{c}]=0$. Применяя лемму 3 к одномерным подпространствам пространства $\mathfrak{c}$, получаем, что $\mathfrak{c}$ - коммутативная подалгебра. Положим теперь $\mathfrak{l}=\mathfrak{c}+\mathfrak{k}+\mathfrak{s} ;$ тогда

$$
\mathfrak{g}=\mathfrak{n} \oplus \mathfrak{l} .
$$

Из односвязности пространства $X$ следует, что $G=N \lambda L$, где $N$ и $L$ - связные подгрупшы Ли с касательными алгебрами $\mathfrak{n}$ и $\mathfrak{l}$ соответственно, причем подгруппа $N$ односвязна и $L \supset K$. Применяя лемму 4 к подгрупе $L$ и подпространству $\mathfrak{n}$, получаем свойство 3).

В силу следствия 3 предложения 10 однородные пространства $L / K$ и $(N \lambda K) / K$ слабо коммутативны. Принимая во внимание, что описание слабо коммутативных однородных пространств редуктивных групп Ли нами уже дано, следуюшим шагом должно быть описание слабо коммутативных однородных пространств вида $(N \lambda K) / K$. Этой проблеме и будет посвяшена оставшаяся часть параграфа.

5. Риманово однородное пространство вида $X=(N \lambda K) / K$, где $N$ - не более чем 2-ступенно нильпотентная односвязная група Ли, будем назьвать пространством гейзенбергова типа.

Если группа $N$ абелева, то $X$ есть просто евклидово пространство с некоторой выделенной группй движений, содержащей все параллельные переносы. Очевидно, что такое однородное пространство слабо коммутативно и даже коммутативно, так как инвариантные дифференциальные операторы на нем - это $K$-инвариантные дифференщиальные операторы с постоянными коэффищиентами.

Дадим критерий слабой коммутативности произвольного однородного пространства гейзенбергова типа.

Пусть $\mathfrak{z}$ - коммутант алгебры Ли $\mathfrak{n}$ (содержащийся в ее центре) и $\mathfrak{v}-$ какое-либо $K$-инвариантное дополнительное подпространство. Для любой линейной функции $\alpha \in \mathfrak{z}^{*}$ обозначим через $\widehat{\alpha}$ кососимметрическую билинейную функщию на $\mathfrak{v}$, определяемую по формуле

$$
\widehat{\alpha}(\xi, \eta)=\alpha([\xi, \eta]) .
$$

Пусть $K_{\alpha}$ - стабилизатор функции $\alpha$ в групе $K$. Естественное линейное представление групшы $K_{\alpha}$ в симплектическом векторном пространстве $\mathfrak{v}_{\alpha}=\mathfrak{v} / \operatorname{Ker} \widehat{\alpha}$ является симплектическим.

Для любой линейной функции $\beta \in(\operatorname{Ker} \widehat{\alpha})^{*}$ обозначим через $K_{\alpha, \beta}$ ее стабилизатор в группе $K_{\alpha}$.

ПРЕДЛОЖЕНИЕ 11. Однородное пространство $X=(N \lambda K) / K$ гейзенбергова типа слабо коммутативно тогда и только тогда, когда для любой линейной функиии $\alpha \in \mathfrak{z}^{*}$ общего положсения и любой линейной функиии $\beta \in(\operatorname{Ker} \widehat{\alpha}){ }^{*}$ общего 
положения естественное линейное действие группь $K_{\alpha, \beta}$ в симплектическом векторном пространстве $\mathfrak{v}_{\alpha}$ является коизотропным.

ДокАЗАТЕЛЬство. Согласно следствию предложения 9 пространство $X$ слабо коммутативно тогда и только тогда, когда для любой линейной функции $\delta \in \mathfrak{n}^{*}$ обшего положения выполнено условие

$$
\delta\left(\left[\left(\operatorname{ad}^{*}(\mathfrak{k}) \delta\right)^{\circ},\left(\operatorname{ad}^{*}(\mathfrak{k}) \delta\right)^{\circ}\right]\right)=0 .
$$

Пусть $\alpha$ - ограничение $\delta$ на $\mathfrak{z}$. Выберем какое-нибудь $K$-инвариантное подпространство $\mathfrak{w}_{\alpha}$, дополнительное к $\operatorname{Ker} \widehat{\alpha}$ в $\mathfrak{v}$. Тогда $\mathfrak{n}^{*}=\mathfrak{z}^{*} \oplus(\operatorname{Ker} \widehat{\alpha})^{*} \oplus \mathfrak{w}_{\alpha}^{*}$ и в соответствии с этим

$$
\delta=\alpha+\beta+\gamma,
$$

где $\beta$ - ограничение $\delta$ на Ker $\widehat{\alpha}$, a $\gamma$ - ограничение $\delta$ на $\mathfrak{w}_{\alpha}$.

Проекция подпространства $\left(\operatorname{ad}^{*}(\mathfrak{k}) \delta\right)^{\circ} \subset \mathfrak{n}$ на $\mathfrak{w}_{\alpha}$ равна $\left(\operatorname{ad}^{*}\left(\mathfrak{k}_{\alpha, \beta}\right) \gamma\right)^{\circ}$, где аннулятор берется в $\mathfrak{w}_{\alpha}$. Поэтому условие $(84)$ переписьвается в следующем виде:

$$
\widehat{\alpha}\left(\left(\operatorname{ad}^{*}\left(\mathfrak{k}_{\alpha, \beta}\right) \gamma\right)^{\circ},\left(\operatorname{ad}^{*}\left(\mathfrak{k}_{\alpha, \beta}\right) \gamma\right)^{\circ}\right)=0
$$

но это и есть условие коизотропности действия группы $K_{\alpha, \beta}$ в $\mathfrak{w}_{\alpha}$ (или в $\mathfrak{v}_{\alpha}$, что все равно).

Выясним теперь, когда симплектическое линейное действие компактной группы Ли является коизотропньм.

Пусть задано линейное представление компактной группы Ли $L$ в вещественном векторном пространстве $V$, сохраняюшее невырожденную 2-форму $\omega$. Продолжим его до линейного представления группы $L(\mathbb{C})$ в пространстве $V(\mathbb{C})$. Полученное представление группы $L(\mathbb{C})$, будучи одновременно симплектическим и ортогональным, разлагается в сумму двух сопряженных друг другу представлений.

Более точно, пространство $V(\mathbb{C})$ может быть разложено в прямую сумму двух $L(\mathbb{C})$-инвариантных изотропных подпространств, двойственных относительно формы $\omega$ :

$$
V(\mathbb{C})=U \oplus U^{*}
$$

(Это разложение, вообще говоря, не единственно.)

ПРЕДЛОЖЕНИЕ 12. Симплектическое линейное действие компактной группь Ли $L$ в вещественном векторном пространстве $V$ коизотропно тогда и только тогда, когда определенное выше действие $L(\mathbb{C}): U$ является сферическим.

ДокАЗАТЕльство. Легко видеть, что действие $L: V$ коизотропно тогда и только тогда, когда действие $L(\mathbb{C}): V(\mathbb{C})$ коизотропно. Ввиду разложения $(85)$ последнее действие можно рассматривать как действие $L(\mathbb{C}): T^{*} U$, и доказьваемое утверждение вытекает из теоремы 2.

Сферических линейных представлений редуктивных алгебраических групп немного, и они все известны [42]-[45], [37]. С учетом этого предложения 11 и 12 дают эффективньй критерий слабой коммутативности однородного пространства гейзенбергова типа.

Точно такой же критерий, но для коммутативности однородного пространства гейзенбергова типа получен в [46] и [47] (см. также [48]). Отсюда вытекает 
Теорема 6. Всякое слабо коммутативное однородное пространство гейзенбергова типа коммутативно.

Независимое доказательство этой теоремы будет дано в следуюшем пункте.

6. Напомним, что алгеброй Вейля $W(V)$ симплектического векторного пространства $(V, \omega)$ назьвается ассоциативная алгебра с единищей, порожденная пространством $V$, с определяюшими соотношениями

$$
x y-y x=\omega(x, y) \quad(x, y \in V) .
$$

Всякое симплектическое преобразование пространства $V$ естественньм образом порождает автоморфизм алгебры $W(V)$.

ПРЕДЛОЖЕНИЕ 13. Симплектическое линейное действие компактной группь Ли $L$ в вещественном пространстве $V$ коизотропно тогда и только тогда, когда алгебра $W(V)^{L}$ коммутативна.

ДокАЗАтЕльство. Алгебра $W(V)$ имеет естественную фильтрацию по степени выражения ее элемента через образуюшие:

$$
W(V)=\bigcup_{n=0}^{\infty} W^{(n)}(V) .
$$

Ассоцированная градуированная алгебра есть симметрическая алгебра $S(V)$. Нетрудно видеть, что

$$
\left[W^{(m)}(V), W^{(n)}(V)\right] \subset W^{(m+n-2)}(V) .
$$

Поэтому, модифицируя конструкцию, изложенную в $\S 4$ главы I, можно превратить алгебру $S(V)$ в градуированную алгебру Пуассона

$$
P(V)=\bigoplus_{n=0}^{\infty} P_{n}(V)
$$

так, что

$$
\left\{P_{m}(V), P_{n}(V)\right\} \subset P_{m+n-2}(V) .
$$

Отождествляя пространство $V$ с сопряженным пространством посредством структурной 2-формы $\omega$, мы можем рассматривать алгебру $S(V)$ как алгебру полиномиальных функций на $V$. Определенная выше скобка Пуассона будет тогда совпадать с обычной скобкой Пуассона в смысле (постоянной) симплектической структуры пространства $V$. В самом деле, в силу тождества Лейбница, справедливого для обеих скобок, достаточно проверить это для линейных функций, т.е. для элементов пространства $V$; но для любых $x, y \in V$ обе скобки равны (константе) $\omega(x, y)$.

Коизотропность действия $L: V$ означает коммутативность алгебры Пуассона $P(V)^{L}$. В соответствии со сказанным выше и в силу полной приводимости линейных представлений групшы $L$ эта алгебра есть не что иное, как градуированная алгебра Пуассона, ассоцированная с фильтрованной ассоциативной алгеброй $W(V)^{L}$. Поэтому если алгебра $W(V)^{L}$ коммутативна, то и алгебра $P(V)^{L}$ коммутативна.

Обратно, пусть действие $L: V$ коизотропно. Рассмотрим разложение (85). Согласно предложению 12 действие $L(\mathbb{C}): U$ является сферическим, т.е. линейное 
представление $L(\mathbb{C}): \mathbb{C}[U]$ имеет простой спектр. Отсюда следует, что алгебра всех линейных операторов в $\mathbb{C}[U]$, перестановочных с операторами этого представления, коммутативна. В частности, алгебра $\mathscr{D}(U)^{L(\mathbb{C})}$ инвариантных дифференциальных операторов с полиномиальными коэффициентами на $U$ коммутативна. Однако $\mathscr{D}(U)=W\left(U \oplus U^{*}\right)=W(V(\mathbb{C}))$ и, значит, $\mathscr{D}(U)^{L(\mathbb{C})}=W(V(\mathbb{C}))^{L(\mathbb{C})}=W(V)^{L}(\mathbb{C})$. Следовательно, алгебра $W(V)^{L}$ коммутативна.

Аналогичное утверждение, по-видимому, справедливо для любой редуктивной алгебраической подгруппы симплектической группы комплексного векторного пространства. По крайней мере, доказательство того, что коммутативность алгебры $W(V)^{L}$ влечет коизотропность действия $L: V$, проходит без изменений в этой ситуации. Доказательство обратной импликации проходит при наличии $L$-инвариантной симметрической билинейной функции в пространстве $V$, но в общем случае оно требует какой-то новой идеи.

ДоКАЗАТЕЛЬСТВо теоремы 6 . Пусть $X=G / K$, где $G=N \lambda K,-$ слабо коммутативное однородное пространство гейзенбергова типа. Докажем, что алгебра $\mathscr{D}(X)^{G}$ инвариантных дифференциальных операторов на $X$ коммутативна.

Пространство $X$ можно отождествить с группой $N$ так, что сама група $N$ будет действовать на нем левыми сдвигами, а группа $K$ - сопряжениями. При этом алгебра $\mathscr{D}(X)^{N}$ будет естественно изоморфна обертьвающей алгебре $U(\mathfrak{n})$ алгебры Ли $\mathfrak{n}$ (см. п. 2.5 главы I), а алгебра $\mathscr{D}(X)^{G}$ - подалгебре $U(\mathfrak{n})^{K} \subset U(\mathfrak{n})$. Таким образом, нам надо доказать коммутативность алгебры $U(\mathfrak{n})^{K}$.

В обозначениях п. 5 алгебру $U(\mathfrak{n})$ можно рассматривать как алгебру сечений расслоения над $\operatorname{Spec} S(\mathfrak{z})=\mathfrak{z}^{*}$, слой которого над точкой $\alpha \in \mathfrak{z}^{*}$ есть алгебра

$$
W_{\alpha}(\mathfrak{v})=U(\mathfrak{n}) /(\xi-\alpha(\xi): \xi \in \mathfrak{z}),
$$

представляющая собой не что иное, как (может быть, вырожденную) алгебру Вейля, определяемую формой $\widehat{\alpha}$ на пространстве $\mathfrak{v}$. Элементам подалгебры $U(\mathfrak{n})^{K}$ будут при этом отвечать $K$-инвариантные сечения. Так как значение $K$-инвариантного сечения в точке $\alpha \in \mathfrak{z}^{*}$ принадлежит подалгебре $W_{\alpha}(\mathfrak{v})^{K_{\alpha}}$, то для доказательства коммутативности алгебры $U(\mathfrak{n})^{K}$ достаточно доказать коммутативность алгебры $W_{\alpha}(\mathfrak{v})^{K_{\alpha}}$ для любой точки $\alpha \in \mathfrak{z}^{*}$ обшего положения.

В свою очередь, если форма $\widehat{\alpha}$ вырожденна, алгебру $W_{\alpha}(\mathfrak{v})$ можно рассматривать как алгебру сечений расслоения над $\operatorname{Spec} S(\operatorname{Ker} \widehat{\alpha})=(\operatorname{Ker} \widehat{\alpha})^{*}$, слой которого над точкой $\beta \in(\operatorname{Ker} \widehat{\alpha})^{*}$ есть алгебра Вейля $W_{\alpha, \beta}\left(\mathfrak{w}_{\alpha}\right)$, определяемая формой $\widehat{\alpha}$ на пространстве $\mathfrak{w}_{\alpha}$. Элементам подалгебры $W_{\alpha}(\mathfrak{v})^{K_{\alpha}}$ будут при этом отвечать $K_{\alpha}$-инвариантные сечения. Поэтому для доказательства коммутативности алгебры $W_{\alpha}(\mathfrak{v})^{K_{\alpha}}$ достаточно доказать коммутативность алгебры $W_{\alpha, \beta}\left(\mathfrak{w}_{\alpha}\right)^{K_{\alpha, \beta}}$ для любой точки $\beta \in$ $(\operatorname{Ker} \widehat{\alpha})^{*}$ общего положения.

Согласно предложению 13 коммутативность алгебры $W_{\alpha, \beta}\left(\mathfrak{w}_{\alpha}\right)^{K_{\alpha, \beta}}$ равносильна коизотропности действия $K_{\alpha, \beta}: \mathfrak{w}_{\alpha}$; но последняя имеет место в силу предложения 11.

7. Вернемся к проблеме классификации (слабо) коммутативных однородных пространств гейзенбергова типа.

Пусть $X=(N \lambda K) / K$ - такое пространство. Будем использовать обозначения п. 5. 
ПРЕДЛОЖЕНИЕ 14. Для любъх инвариантов $\varphi, \psi \in \mathbb{R}[\mathfrak{v}]^{K}$ и любой точки $a \in \mathfrak{v}$ выполняется соотношение

$$
\left[\operatorname{grad}_{a} \varphi, \operatorname{grad}_{a} \psi\right]=0
$$

ДокаЗАТЕЛЬСтво. Можно считать, что $\mathfrak{v} \perp \mathfrak{z}$. Рассматривая $\varphi$ и $\psi$ как инварианты на $\mathfrak{n}$ и записывая соотношение (82) для их дифференциалов в точке $a+b(a \in \mathfrak{v}$, $b \in \mathfrak{z})$, получаем:

$$
\left(b,\left[\operatorname{grad}_{a} \varphi, \operatorname{grad}_{a} \psi\right]\right)=0 .
$$

Так как $b$ может быть любым элементом из $\mathfrak{z}$, то отсюда следует (86).

Рассмотрим какое-либо разложение пространства $\mathfrak{v}$ в прямую сумму минимальных $K$-инвариантных подпространств:

$$
\mathfrak{v}=\mathfrak{v}_{1} \oplus \cdots \oplus \mathfrak{v}_{s}
$$

ПРЕДЛОЖЕНИЕ 15. 1) $\left[\mathfrak{v}_{i}, \mathfrak{v}_{j}\right]=0$ nрu $i \neq j$.

2) Если $K$-модули $\mathfrak{v}_{i}$ u $\mathfrak{v}_{j}(i \neq j)$ изоморфньи, то $\left[\mathfrak{v}_{i}, \mathfrak{v}_{i}\right]=\left[\mathfrak{v}_{j}, \mathfrak{v}_{j}\right]=0$.

ДокАЗАТЕЛЬСТво. Первое утверждение получается применением леммы 3 к подпространствам $\mathfrak{v}_{i}$ и $\mathfrak{v}_{j}$. Второе утверждение получается с учетом первого применением той же леммы к другим минимальным инвариантным подпространствам суммы $\mathfrak{v}_{i} \oplus \mathfrak{v}_{j}$, например, к $\mathfrak{v}_{i}$ и диагонали этой суммы.

Пространство $X$ назовем неприводимым, если представление $K: \mathfrak{v}$ неприводимо, т.e. $s=1$.

В общем случае для любого $i=1, \ldots, s$ можно рассмотреть $K$-инвариантную подалгебру

$$
\mathfrak{n}_{i}=\mathfrak{v}_{i}+\left[\mathfrak{v}_{i}, \mathfrak{v}_{i}\right] \subset \mathfrak{n}
$$

и соответствуюшую ей связную подгруппу $N_{i}$ группы $N$; тогда в силу следствия 3 предложения 10 однородное пространство $X_{i}=\left(N_{i} \lambda K\right) / K$ также коммутативно (но группа $K$ может действовать на нем не эффективно). Назовем его неприводимой компонентой пространства $X$. Предложение 15 показьвает, что пространство $X$ достаточно просто (но все же, быть может, не тривиально) составляется из своих неприводимых компонент.

Далее, если zо $\subset \mathfrak{z}$ - какое-либо $K$-инвариантное подпространство и $Z_{0} \subset Z$ - соответствующая связная подгруппа, то в силу следствия 1 предложения 10 однородное пространство $X / Z_{0}=\left(\left(N / Z_{0}\right) \lambda K\right) / K$ также коммутативно. Переход от $X$ к $X / Z_{0}$ назовем иентральной редукиией. Коммутативное однородное пространство $X$ гейзенбергова типа назовем максимальжым, если оно не может быть получено нетривиальной центральной редукцией из большего коммутативного однородного пространства гейзенбергова типа.

В таблице 3 перечислены все максимальные неприводимые коммутативные однородные пространства гейзенбергова типа с неабелевой группой $N$ (т.е. с $\mathfrak{z} \neq 0$ ) с точностью до возможной замены группы $\mathrm{SU}_{n}$ на $\mathrm{U}_{n}$ во всех случаях, где эта группа встречается. При этом используются следуюшие обозначения:

$\mathbb{H}$ - алгебра кватернионов;

$\mathbb{H}_{0}-$ пространство чисто мнимых кватернионов; 
$\mathbb{C}^{m} \otimes \mathbb{H}^{n}\left(\right.$ и $\left.\mathbb{H}^{n} \otimes \mathbb{C}^{m}\right)$ - тензорное произведение над $\mathbb{C} ;$

$\mathbb{H}^{m} \otimes \mathbb{H}^{n}$ - тензорное произведение над $\mathbb{H}$;

$H S^{2}-$ эрмитов квадрат;

$H \Lambda^{2}-$ косоэрмитов квадрат;

$H S_{0}^{2} \mathbb{H}^{n}$ - пространство эрмитовых кватернионных матриц порядка $n$ с нулевьм следом.

ТАБЛИЦА 3

(Все группы - вещественные.)

\begin{tabular}{|c|c|c|c|c|}
\hline & $K$ & $\mathfrak{v}$ & $\mathfrak{z}$ & \\
\hline 1 & $\mathrm{SO}_{n}$ & $\mathbb{R}^{n}$ & $\Lambda^{2} \mathbb{R}^{n}$ & \\
\hline 2 & $\mathrm{U}_{1} \times \mathrm{SO}_{n}$ & $\mathbb{C}^{n}$ & $\mathbb{R}$ & \\
\hline 3 & $\mathrm{SU}_{n}$ & $\mathbb{C}^{n}$ & $\Lambda^{2} \mathbb{C}^{n}$ & $n$ нечетно \\
\hline 4 & $\mathrm{SU}_{n}$ & $\mathbb{C}^{n}$ & $\mathbb{R}$ & $n$ нечетно \\
\hline 5 & $\mathrm{SU}_{n}$ & $\mathbb{C}^{n}$ & $\Lambda^{2} \mathbb{C}^{n} \oplus \mathbb{R}$ & $n$ четно \\
\hline 6 & $\mathrm{U}_{n}$ & $\mathbb{C}^{n}$ & $H \Lambda^{2} \mathbb{C}^{n}$ & \\
\hline 7 & $\mathrm{SU}_{2} \times \mathrm{SU}_{n}$ & $\mathbb{C}^{2} \otimes \mathbb{C}^{n}$ & $H \Lambda^{2} \mathbb{C}^{2}$ & \\
\hline 8 & $\mathrm{Sp}_{2} \times \mathrm{SU}_{n}$ & $\mathbb{H}^{2} \otimes \mathbb{C}^{n}$ & $\mathbb{R}$ & $n \geqslant 5$ \\
\hline 9 & $\mathrm{Sp}_{2} \times \mathrm{U}_{n}$ & $\mathbb{H}^{2} \otimes \mathbb{C}^{n}$ & $\mathbb{R}$ & $n \geqslant 4$ \\
\hline 10 & $\mathrm{SU}_{m} \times \mathrm{SU}_{n}$ & $\mathbb{C}^{m} \otimes \mathbb{C}^{n}$ & $\mathbb{R}$ & \\
\hline 11 & $\mathrm{Sp} n$ & $\mathbb{H}^{n}$ & $H S_{0}^{2} \mathbb{H}^{n} \oplus \mathbb{H}_{0}$ & \\
\hline 12 & $\mathrm{U}_{1} \times \mathrm{Sp}_{n}$ & $\mathbb{H}^{n}$ & $H S_{0}^{2} \mathbb{H}^{n} \oplus \mathbb{H}_{0}$ & \\
\hline 13 & $\mathrm{U}_{2} \times \mathrm{Sp}_{n}$ & $\mathbb{C}^{2} \otimes \mathbb{H}^{n}$ & $H \Lambda^{2} \mathbb{C}^{2}$ & \\
\hline 14 & $\mathrm{U}_{3} \times \mathrm{Sp} n$ & $\mathbb{C}^{3} \otimes \mathbb{H}^{n}$ & $\mathbb{R}$ & \\
\hline 15 & $\mathrm{Sp}_{1} \times \mathrm{Sp}_{n}$ & $\mathbb{H}^{n}$ & $\mathbb{H}_{0}$ & \\
\hline 16 & $\mathrm{Sp}_{2} \times \mathrm{Sp}_{n}$ & $\mathbb{H}^{2} \otimes \mathbb{H}^{n}$ & $H \Lambda^{2} \mathbb{H}^{2}$ & \\
\hline 17 & $\mathrm{U}_{n}$ & $S^{2} \mathbb{C}^{n}$ & $\mathbb{R}$ & \\
\hline 18 & $\mathrm{U}_{n}$ & $\Lambda^{2} \mathbb{C}^{n}$ & $\mathbb{R}$ & \\
\hline 19 & $\mathrm{SU}_{n}$ & $\Lambda^{2} \mathbb{C}^{n}$ & $\mathbb{R}$ & $n$ нечетно \\
\hline 20 & $\operatorname{Spin}_{7}$ & $\mathbb{R}^{8}$ & $\mathbb{R}^{7}$ & \\
\hline 21 & $\mathrm{U}_{1} \times \operatorname{Spin}_{7}$ & $\mathbb{C}^{8}$ & $\mathbb{R}$ & \\
\hline 22 & $\mathrm{U}_{1} \times \mathrm{Spin}_{9}$ & $\mathbb{C}^{16}$ & $\mathbb{R}$ & \\
\hline 23 & $\operatorname{Spin}_{10}$ & $\mathbb{C}^{16}$ & $\mathbb{R}$ & \\
\hline 24 & $\mathrm{U}_{1} \times \operatorname{Spin}_{10}$ & $\mathbb{C}^{16}$ & $\mathbb{R}$ & \\
\hline 25 & $\mathrm{U}_{1} \times \mathrm{E}_{6}$ & $\mathbb{C}^{27}$ & $\mathbb{R}$ & \\
\hline 26 & $\mathrm{G}_{2}$ & $\mathbb{R}^{7}$ & $\mathbb{R}^{7}$ & \\
\hline 27 & $\mathrm{U}_{1} \times \mathrm{G}_{2}$ & $\mathbb{C}^{7}$ & $\mathbb{R}$ & \\
\hline
\end{tabular}

Операция коммутирования $\mathfrak{v} \times \mathfrak{v} \rightarrow \mathfrak{z}$ во всех случаях однозначно определяется условием $K$-эквивариантности. Например, в строках 11 и 12 коммутатор столбцов 
$x, y \in \mathbb{H}^{n}=\mathfrak{v}$ задается формулой

$$
[x, y]=\left(\left(x i y^{*}-y i x^{*}\right)-\frac{1}{n}\left(\operatorname{Re} i\left(y^{*} x-x^{*} y\right)\right) E, y^{*} x-x^{*} y\right),
$$

где звездочка обозначает транспонирование, совмещенное с кватернионньм сопряжением, а $E$ - единичная матрища.

Метод получения этой таблицы состоит в обших чертах в следующем. Предложение 14 показьвает, что если неприводимое представление $K: \mathfrak{v}$ настолько велико, что внешние произведения $\operatorname{grad}_{a} \varphi \wedge \operatorname{grad}_{a} \psi\left(\varphi, \psi \in \mathbb{R}[\mathfrak{v}]^{K}, a \in \mathfrak{v}\right)$ порождают пространство $\Lambda^{2} \mathfrak{v}$, то $\mathfrak{z}=0$. Это соображение оставляет сравнительно небольшое число представлений, которые исследуются индивидуально. Детали доказательства будут опубликованы в другом месте.

Отметим, что классификация коммутативных однородных пространств гейзенбергова типа с $\operatorname{dim} \mathfrak{z}=1$ (сводящаяся ввиду вьшеизложенного к классификации сферических представлений) была получена в [46] и [44]. Другой класс коммутативных однородных пространств гейзенбергова типа описан в [49].

Анализируя таблицу 3 , можно показать, что почти все неприводимые коммутативные однородные пространства гейзенбергова типа являются слабо симметрическими. Исключение составляют лишш пространства из строк 11 и 12 и некоторые их центральные редукщии. В числе последних серия пространств с

$$
K=\mathrm{Sp}_{n}, \quad \mathfrak{v}=\mathbb{H}^{n}, \quad \mathfrak{z}=\mathbb{H}_{0}
$$

Это были первые примеры не слабо симметрических коммутативных однородных пространств, построенные в [18].

\section{СПИСОК ЛИТЕРАТУРЫ}

[1] Э. Б. Винберг, А. Л. Онищик. Семинар по группам Ли и алгебраическим группам. М.: Наука, 1988.

[2] Э. Б. Винберг, В. Л. Попов. Теория инвариантов // Итоги науки и техники. Совр. пробл. матем. Фунд. напр. Т. 55. М.: ВИНИТИ, 1989. С. 137-309.

[3] S. Helgason. Differential Geometry, Lie Groups, and Symmetric Spaces. London: Academic Press, 1978.

[4] A. Selberg. Harmonic analysis and discontinuous groups in weakly symmetric Riemannian spaces with applications to Dirichlet series // J. Indian Math. Soc. 1956. V. 20. P. 47-87.

[5] J. Berndt, F. Prüfer, L. Vanhecke. Symmetric-like Riemannian manifolds and geodesic symmetries // Proc. Roy. Soc. Edinburgh Sect. A. 1995. V. 125. P. 265-282.

[6] S. Nagai. Weakly symmetric spaces in complex and quaternionic space forms // Arch. Math. 1995. V. 65. P. 342-351.

[7] J. Berndt, L. Vanhecke. Geometry of weakly symmetric spaces // J. Math. Soc. Japan. 1996. V. 48. P. 745-760.

[8] O. Kowalski, F. Prüfer, L. Vanhecke. D'Atri Spaces // Topics in Geometry: Honoring the Memory of Joseph D'Atri / ed. S. Gindikin. Boston: Birkhäuser, 1996. P. 241-284.

[9] W. Ziller. Weakly symmetric spaces // Topics in Geometry: Honoring the Memory of Joseph D'Atri / ed. S. Gindikin. Boston: Birkhäuser, 1996. P. 355-368.

[10] H. Nguyễ̃. Weakly symmetric spaces and bounded symmetric domains // Transformation Groups. 1997. V. 2. P. 351-374.

[11] А. А. Кириллов. Введение в теорию представлений и некоммутативньй гармонический анализ // Итоги науки и техники. Совр. пробл. матем. Фунд. напр. Т. 22. М.: ВИНИТИ, 1988. C. $5-162$. 
[12] Ф. А. Березин, И. М. Гельфанд, М. И. Граев, М. А. Наймарк. Представление групп // УМН. 1956. Т. 11. №6. С. 13-40.

[13] И. М. Гельфанд. Сферические функции на симметрических римановых пространствах // Докл. АН СССР. 1950. Т. 70. С. 5-8.

[14] Н. Бурбаки. Интегрирование. М.: Наука, 1967.

[15] H. Grauert. On Levi's problem and the imbedding of real-analytic manifolds // Ann. Math. (2). 1958. V. 68. P. 460-472; // Математика 1960. T. 4. №3. C. 29-40.

[16] С. Хелгасон. Группы и геометрический анализ. М.: Мир, 1987.

[17] E. G. F. Thomas. An infinitesimal characterization of Gelfand pairs // Contemp. Math. 1984. V. 26. P. 379-385.

[18] J. Lauret. Commutative spaces which are not weakly symmetric // Bull. London Math. Soc. 1998. V. 30. P. 29-36.

[19] К. Шевалле. Теория групп Ли. Т. ІІІ. М.: ИЛ, 1958.

[20] Э. Б. Винберг, Б. Н. Кимельфельд. Однородные области на флаговых многообразиях и сферические подгруппы простых групп Ли // Функц. анализ и его прил. 1978. Т. 12. №1. C. $12-19$.

[21] M. Krämer. Sphärische Untergruppen in kompakten zusammenhängenden Liegruppen // Compositio Math. 1979. V. 38. P. 129-153.

[22] И. В. Микитюк. Об интегрируемости инвариантных гамильтоновых систем с однородными конфигурационными пространствами // Матем. сб. 1986. Т. 129. С. 514-534.

[23] M. Brion. Classification des espaces homogènes sphériques // Compositio Math. 1987. V. 63. P. $189-208$.

[24] E. Vinberg. On stability of actions of reductive algeabraic groups // Lie Algebras, Rings and Related Topics / ed. Y. Fong, A. A. Mikhalev, E. Zelmanov. Berlin: Springer-Verlag, 2000. P. 188-202.

[25] D. N. Akhiezer, E. B. Vinberg. Weakly symmetric spaces and spherical varieties // Transformation Groups. 1999. V. 4. P. 3-24.

[26] R. Steinberg. Endomorphisms of Algebraic Groups. Providence, RI: Amer. Math. Soc., 1968. (Mem. Amer. Math. Soc. V. 80.)

[27] Э. Б. Винберг, В.В.Горбацевич, А. Л. Онищик. Строение групп и алгебр Ли // Итоги науки и техники. Совр. пробл. матем. Фунд. напр. Т. 41. М.: ВИНИТИ, 1990. C. $5-253$.

[28] V. Guillemin, S. Sternberg. Multiplicity-free spaces // J. Differential Geom. 1984. V. 19. P. $31-56$.

[29] В. И. Арнольд, А. Б. Гивенталь. Симплектическая геометрия // Итоги науки и техники. Совр. пробл. матем. Фунд. напр. Т. 4. М.: ВИНИТИ, 1985. С. 5-139.

[30] Б. А. Дубровин, И. М. Кричевер, С. П. Новиков. Интегрируемые системы. I // Итоги науки и техники. Совр. пробл. матем. Фунд. напр. Т. 4. М.: ВИНИТИ, 1985. C. $179-285$.

[31] В. В. Трофимов, А. Т. Фоменко. Алгебра и геометрия интегрируемых гамильтоновых дифференциальных уравнений. М.: МГУ, 1995.

[32] W. Borho, H. Kraft. Über Bahnen und deren Deformationen bei linearen Aktionen reduktiver Gruppen // Comment. Math. Helv. 1979. V. 54. P. 61-104.

[33] F. Knop. Weylgruppe und Momentabbildung // Invent. Math. 1990. V. 99. P. 1-23.

[34] M. Brion, D. Luna, T. Vust. Espaces homogènes sphériques // Invent. Math. 1986. V. 84. P. 617-632.

[35] F. D. Grosshans. Constructing invariant polynomials via Tschirnhaus transformations // Lecture Notes in Math. 1985. V. 1278. P. 95-102.

[36] F. Knop. The asymptotic behavior of invariant collective motion // Invent. Math. 1994. V. 116. P. 309-328.

[37] F. Knop. Some remarks on multiplicity free spaces // Representation Theories and Algebraic Geometry / ed. A. Broer, A. Daigneault. Dordrecht: Kluwer, 1998. P. 301-317.

[38] H. Sumihiro. Equivariant completion // J. Math. Kyoto Univ. 1974. V. 14. P. 1-28.

[39] T. Kambayashi. Projective representation of algebraic linear groups of transformations // Amer. J. Math. 1966. V. 88. P. 199-205. 
[40] F. Knop. Über Bewertungen, welche unter einer reduktiven Gruppe invariant sind // Math. Ann. 1993. V. 295. P. 333-363.

[41] И. М. Гельфанд, М. И. Граев. Геометрия однородных пространств, представления групп в однородных пространствах и связанные с ними вопросы интегральной геометрии. I // Труды MМO. 1959. Т. 8. C. 321-390.

[42] V. Kac. Some remarks on nilpotent orbits // J. Algebra. 1980. V. 64. P. 190-213.

[43] M. Brion. Représentations exceptionelles des groupes semi-simples // Ann. Sci. École Norm. Sup. (4). 1985. V. 18. P. 345-387.

[44] C. Benson, G. Ratkliff. A classification of multiplicity free actions // J. Algebra. 1996. V. 181. P. 152-186.

[45] A. Leahy. A classification of multiplicity free representations // J. Lie Theory. 1998. V. 8. P. $367-391$.

[46] C. Benson, J. Jenkins, G. Ratkliff. On Gelfand pairs associated with solvable Lie groups // Trans. Amer. Math. Soc. 1990. V. 321. P. 85-116.

[47] K. Kikuchi. On Gelfand pairs associated with nilpotent Lie groups // J. Math. Kyoto Univ. 1994. V. 34. P. 741-754.

[48] C. Benson, J. Jenkins, G. Ratkliff. The orbit method and Gelfand pairs associated with nilpotent Lie groups // J. Geom. Anal. 1999. V. 9. P. 569-582.

[49] J. Lauret. Gelfand pairs attached to representations of compact Lie groups // Transformation Groups. 2000. V. 5. P. 307-324.

Московский государственньй

Поступила в редакцию

университет им. М. В. Ломоносова

12.10 .2000 